Florida International University FIU Digital Commons

$6-5-2014$

\title{
Influence of Gender and Life Stressors on Longitudinal Depression Treatment Outcomes Among Older Primary Care Patients
}

Karen Fortuna

klfortuna@gmail.com

DOI: $10.25148 /$ etd.FI14071108

Follow this and additional works at: https://digitalcommons.fiu.edu/etd

\section{Recommended Citation}

Fortuna, Karen, "Influence of Gender and Life Stressors on Longitudinal Depression Treatment Outcomes Among Older Primary Care Patients" (2014). FIU Electronic Theses and Dissertations. 1444.

https://digitalcommons.fiu.edu/etd/1444 


\section{FLORIDA INTERNATIONAL UNIVERSITY}

Miami, Florida

\section{INFLUENCE OF GENDER AND LIFE STRESSORS ON LONGITUDINAL DEPRESSION TREATMENT OUTCOMES AMONG OLDER PRIMARY CARE \\ PATIENTS}

A dissertation submitted in partial fulfillment of

the requirements for the degree of

DOCTOR OF PHILOSOPHY

in

SOCIAL WELFARE

by

Karen Fortuna

2014 
To: Dean Michele Ciccazzo

R. Stempel College of Public Health and Social Work

This dissertation, written by Karen Fortuna, and entitled Influence of Gender and Life Stressors on Longitudinal Depression Treatment Outcomes Among Older Primary Care Patients, having been approved in respect to style and intellectual content, is referred to you for your judgment.

We have read this dissertation and recommend that it be approved.

$\begin{array}{r}\hline \text { Nicole Ruggiano } \\ \hline \text { Frank Dillon } \\ \hline \text { Florence Keane } \\ \hline \text { Cynthia Zubritsky } \\ \hline \text { Barbara Thomlison, Major Professor }\end{array}$

Date of Defense: June 5, 2014

The dissertation of Karen Fortuna is approved.

Dean Michele Ciccazzo

R. Stempel College of Public Health and Social Work

Dean Lakshmi N. Reddi University Graduate School

Florida International University, 2014 


\section{DEDICATION}

I would like to dedicate this to David Fortuna. You have taught me how to persevere in the face of adversity to achieve my dreams. You made me the person I am today, and I forever thank you. I would also like to dedicate this to Mark Whiteman and Cynthia Zubritsky who have both provided me more support and encouragement over the past few years than my words can express. I would not be at this point in my life without the two of you (10/27).

Thank you all. 


\section{ACKNOWLEDGMENTS}

The guidance and expertise of Dr. Barbara Thomlison made this dissertation possible. I was honored to have the opportunity to work with Dr. Thomlison as my advisor. I graciously thank her for working with me and seeing me through this process. I was so fortunate to work with Dr. Thomlison. I cannot thank you enough for working with me or express the level of gratitude I have in words.

I would also like to thank Dr. Frank Dillon for your support from the first day of the program until now and never giving up on me. I would also like to thank and extend my gratitude to Dr. Nicole Ruggiano who has mentored me, inspired me, and has taught me how represent the field of gerontological social work.

I would like to extend my gratitude to Dr. Florence Keane for her involvement on the committee. Her skills and expertise were greatly appreciated in the development of this dissertation.

I would like to thank Dr. Cynthia Zubritsky who has supported me throughout this process through countless phone calls, emails, and visits to Philadelphia. I am so fortunate to have met you and to have worked with you. Your tutelage inspired me to pursue my $\mathrm{PhD}$ and focus my studies on mental health and older adults. I cannot thank you enough for the mentorship you have provided me over these past nine years.

I would also like to thank David Saltman, who has supported me and taught me about the field of gerontological social work. I am thankful for the opportunity to have 
met David through this program and look forward to future conversations about the behavioral health needs of older adults. 


\section{ABSTRACT OF THE DISSERTATION \\ INFLUENCE OF GENDER AND LIFE STRESSORS ON LONGITUDINAL DEPRESSION TREATMENT OUTCOMES AMONG OLDER PRIMARY CARE \\ PATIENTS \\ by}

Karen Fortuna

Florida International University, 2014

Miami, Florida

Professor Barbara Thomlison, Major Professor

Purpose: Depression in older females is a significant and growing problem. Females who experience life stressors across the life span are at higher risk for developing problems with depression than their male counterparts. The primary aim of this study was (a) to examine gender-specific differences in the correlates of depression in older primary care patients based on baseline and longitudinal analyses; and (b) to examine the longitudinal effect of biopsychosocial risk factors on depression treatment outcomes in different models of behavioral healthcare (i.e., integrated care and enhanced referral). Method: This study used a quantitative secondary data analysis with longitudinal data from the Primary Care Research in Substance Abuse and Mental Health for Elderly (PRISM-E) study. A linear mixed model approach to hierarchical linear modeling was used for analysis using baseline assessment, and follow-up from three-month and six-month. Results: For participants diagnosed with major depressive disorder female gender was associated with increased depression severity at six-month compared to males at six-month. Further, the interaction between gender and life stressors found that females who reported loss of family and 
friends, family issues, money issues, medical illness was related to higher depression severity compared to males whereas lack of activities was related to lower depression severity among females compared to males. Conclusion: These findings suggest that gender moderated the relationship between specific life stressors and depression severity similar to how a protective factor can impact a person's response to a problem and reduce the negative impact of a risk factor on a problem outcome. Therefore, life stressors may be a reliable predictor of depression for both females and males in either behavioral health treatment model. This study concluded that life stressors influence males basic comfort, stability, and survival whereas life stressors influence females' development, personal growth, and happiness; therefore, life stressors may be a useful component to include in gender-based screening and assessment tools for depression. 


\section{TABLE OF CONTENTS}

CHAPTER

PAGE

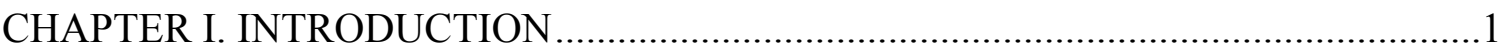

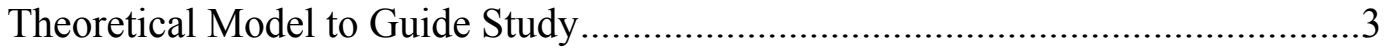

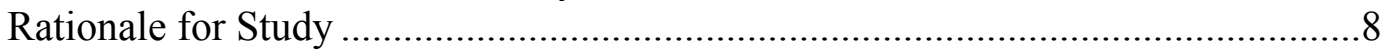

CHAPTER II. LITERATURE REVIEW …........................................................... 12

Risk Factors for Depression in Older Females ................................................15

Psychological and Biological Risk Factors for Older Females for Depression.....20

Research Questions and Hypotheses ...............................................................22

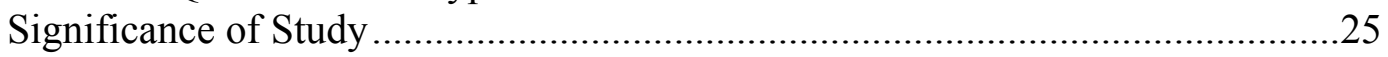

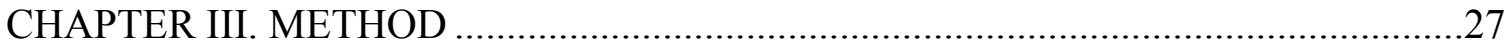

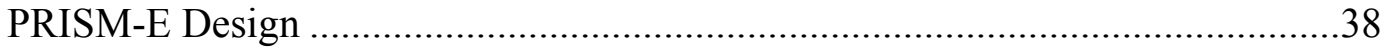

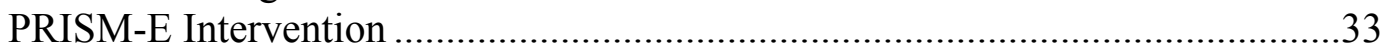

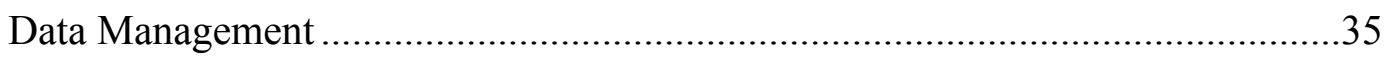

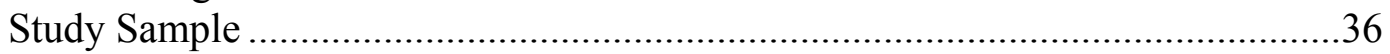

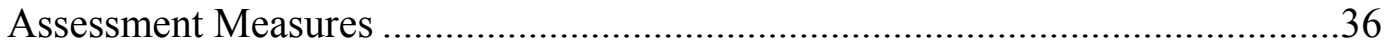

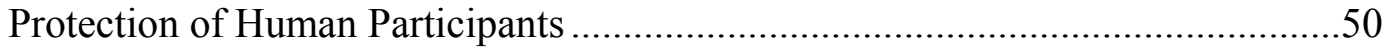

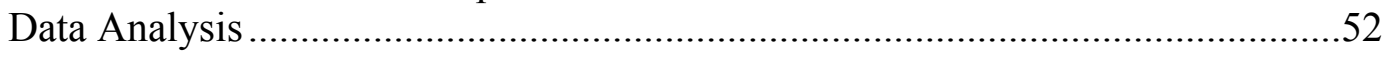

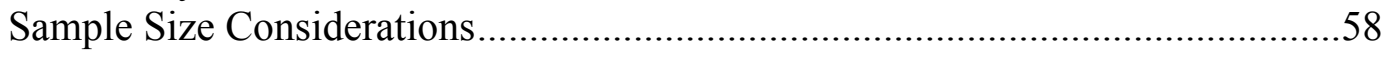

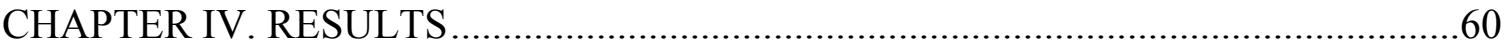

Testing For Assumptions Of Parametric Tests ...................................................60

Data Transformation ...............................................................................6

Descriptive Statistics....................................................................................63

Hierarchical Linear Modeling: Linear Mixed Model Approach...........................64

Correlation Matrix and Variance-Inflation Factor Results ................................65

Building Hierarchical Linear Models Using Linear Mixed Model Approach.......67

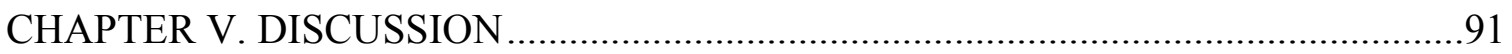

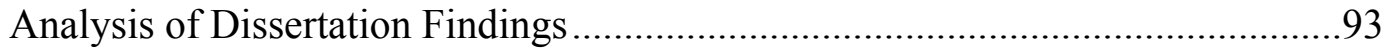

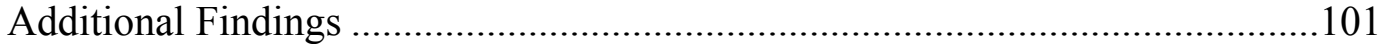

Gender-Based Risk and Resilience ..............................................................101

Implications for Social Work and Public Health ............................................. 102

Integrated Life Span Risk and Resilience Perspective ....................................107

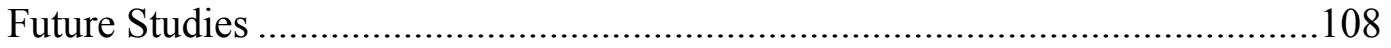

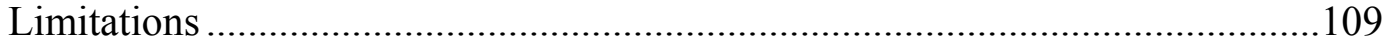

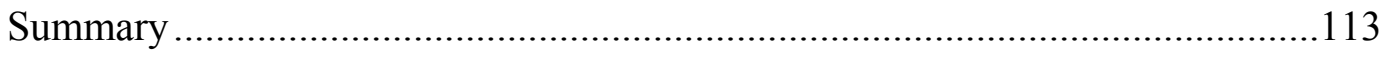

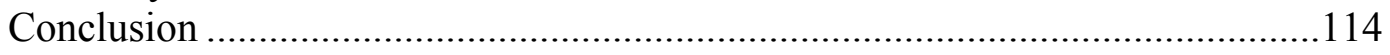

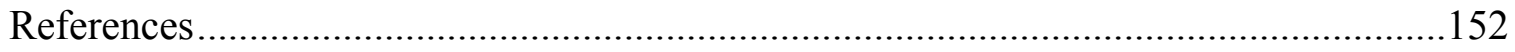




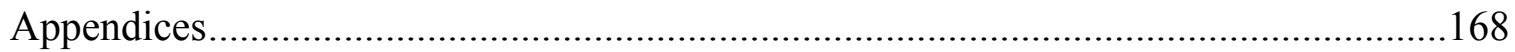

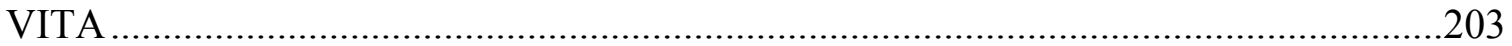




\section{LIST OF TABLES}

TABLE

PAGE

1. Characteristics of Primary Care Settings Involved in the Primary Care Research in Substance Abuse and Mental Health for the Elderly study. ...

2. Characteristics of Non-Veterans Administration Primary Care Settings Involved in the Primary Care Research in Substance Abuse and Mental Health for the Elderly study

3. Characteristics of Integrated Care and Enhanced Specialty Referral Treatment for Major Depressive Disorder and Dysthymia.

4. Baseline Characteristics of Participants in the PRISM-E Study

5. Baseline Characteristics of Variables of Interest of Participants in the PRISM-E Study.

6. Changes in Depression Severity From Baseline, Three-Month, and Six-Month Follow-Up.

7. Correlation Matrix of Variables in the Enhanced Specialty Referral Model.

8. Collinearity Statistics for Enhanced Specialty Referral Model.

9. Correlation Matrix of Variables in the Integrated Care Model

10. Collinearity Statistics for Integrated Care Model.

11. Akaike's Information Criterion (AIC) and Bayesian Information Criterion (BIC)

For Each Hierarchical Linear Model

12. Differences in Average Scores on the CES-D for Participants Across Levels of Depression

13. Impact of The Frequency of Life Stressors on Baseline, Three-Month, and Six Month Scores on the CES-D Among Participants Across Levels of Depression

14. Impact of Gender on Baseline, Three-month, and Six-Month Scores on the CES-D Among Participants Across Levels of Depression.

15. Impact of the Interaction Between Gender and Frequency of Life Stressors on Baseline, Three-Month, and Six-Month Scores on the CES-D Among 
Participants Across Levels of Depression 


\section{CHAPTER I. INTRODUCTION}

Depression in older adults reduces quality of life and is a risk factor for suicide (Centers for Diease Control and Prevention [CDC], 2005) and other physical (Blazer, 2003; Bruce, 2001; Hays et al., 1997; Kraaij et al., 2002; Magaziner et al., 1990; Robinson \& Price, 1982; Sullivan et al., 1997) and behavioral health problems (Blazer, 2009; Cuijpers, van Straten, \& Warmerdam, 2007; Dupree, Broskowski, \& Schonfeld, 1984; Glass et al., 1995; Schonfeld et al., 2010; Thomas \& O’Brien, 2006). Adults aged 65 years and older are projected to increase dramatically over the next 30 years (Vincent \& Velkoff, 2010). In the United States, females have a longer life expectancy than men, 81.1 years and 76.3 years, respectively (Hoyert \& Xu, 2012). Gender differences in life expectancy have resulted in a larger proportion of females compared to males in the older age cohorts (Vincent \& Velkoff, 2010). In 2050 population projections suggest that 55\% of adults aged 65 years and older are estimated to be female while $61 \%$ of adults aged 85 years and older are estimated to be female in 2050 (Vincent \& Velkoff, 2010). The rapid growth of the female aging population will make new demands on the health care system in the United States. Specifically, females are at-risk for depression from youth to older adulthood, and according to Burt and Stein (2002) "females have a lifetime rate of major depressive disorder 1.7 to 2.7 times greater than men" (p. 258).

The severity and frequency of depression and other symptoms among females compared to males vary across studies (Fiske, Gatz, \& Pedersen, 2009; Umberson, Wortman \& Kessler, 1992). Also some studies have found older females endorse different symptoms of depression compared to older males (Kockler \& Heun, 2002; 
Boswell \& Stoudemire, 1996). Research has focused primarily on identifying and reducing the symptoms of depression for the general aging population; however, there are substantial limitations to the symptom-based approach to reducing depression due to gender-specific differences including (a) little consensus among researchers of a viable theoretical framework by which to study gender-specific development of depression; (b) measurement of symptomology offers little insight into long-term impact of resilience; (c) current trait-based instruments designed to assess particular diagnostic criteria have limited utility because they fail to distinguish the deviant developmental process related to gender as it unfolds and tend to identify only the more extreme cases.

Characterizing depression using the symptom-based approach ignores examining the foundational causes of depression in a person's development and the risk factors they have been exposed to in their life span. In social work, human behavior is the result of interactions between individuals and their environment (Wormer, 2007). Therefore, to move away from the limited symptoms-based approach to depression, there is a need to examine gender-specific differences in the correlates of depression more holistically where the interactions between individuals and their environment are considered.

Specifically for older females, co-occurring problems and life stressors can worsen depression symptoms (Kessler, 2003; Nolen-Hoeksema et al., 1999). Evidence suggests a closer link between co-occurring paths of life stressors and depression for females than males (Kessler, 2003; Nolen-Hoeksema et al., 1999). To examine genderbased differences, an examination of the influence of life stressors on the development of depression may assist in addressing the different developmental pathways of depression 
for older females, and impact their quality of life and help reduce the societal costs of depression.

To fill the gap in knowledge on gender-specific differences in the correlates of depression and inform screening and early preventative interventions, this study aimed to: (a) to examine gender-specific differences in the correlates of depression in older primary care patients; and (b) to examine the longitudinal effect of biopsychosocial risk factors on depression treatment outcomes in different models of behavioral health care (i.e., integrated care and enhanced referral). This study used a quantitative secondary data analysis with longitudinal data from the Primary Care Research in Substance Abuse and Mental Health for Elderly (PRISM-E) study.

\section{Theoretical Model to Guide Study}

The theoretical framework used to guide this study was based on the integration of two existing perspectives: the life span perspective (Baltes, 1987; Baltes, Lindenberger, \& Staudinger, 2006) and the risk and resilience perspective (Rutter, 1987; Saleebey, 1996). The following is a description of each theoretical perspective, and a description of the integration of these two perspectives as applied to this study. The two models have been combined to develop a viable alternative to the traditional stage based theories of human development across the life span (Bijou, 1993). In addition, in social work there is a fundamental assumption that human behavior is the result of the interactions between the individual and their environment (Wormer, 2007). They reciprocally shape each other - there are continual influences each within a particular context - thus some environments are more risk charged than others, and others more 
protective. This encompasses the reciprocal influences between the individual, the relevant others, and the social environment.

The life span perspective. As person's life expectancy has increased (Santrock, 2011) and the aging population is increasing in the United States (Vincent \& Velkoff, 2010), the life span perspective has become more important in micro practice intervention design, development, and evaluation. The life span perspective suggests that how a person develops is part of interactions between biological, sociocultural, and individual processes experienced throughout a life span (Baltes, 1987; Baltes, Lindenberger, \& Staudinger, 2006).

The life span perspective has several advantages over traditional theories of human development. The life span perspective suggests that development occurs throughout a lifetime (Baltes, 1987; Baltes, Lindenberger, \& Staudinger, 2006; Santrock, 2011). The well established approach to the study of development focuses on birth, adolescence, and decline in older adulthood (Santrock, 2011). However, the life span perspective emphasizes developmental change throughout the life span (Blazer \& Steffens, 2009; Santrock, 2011). Therefore, in older adulthood, individuals are still developing, and their development is a product of dynamic interactions between the developing self and the environment throughout the entirety of the life span (Blazer \& Steffens, 2009; Santrock, 2011). These interactions can have direct and indirect effects on the development of depression. For example, an older woman may experience a life stressor such as moving into a nursing home. The move directly affects the older female by physically changing her location. The woman is indirectly affected as she is no longer 
living in her familiar community with her friends and neighbors. The staff at the nursing home can no longer meet her social and emotional needs, which may result in social isolation and symptoms of depression.

These dynamic interactions are also influenced by a person's gender since human beings do not experience universally fixed, sequential stages of development (Gitterman, 2011); rather, gender and historical context have a profound influence on individuals and their collective development (Gitterman, 2011). Consistent with research on development, life stressors in older adulthood have a significant relationship with depression (Kraaij et al., 2002), particularly for older females (Kessler, 2003). Research has suggested that females respond differently to life stressors compared to men making them more vulnerable to depression (Fiske et al., 2009; Nolen-Hoeksema et al., 1999). For example, females have a greater likelihood of developing depression or depressive symptoms after the death of a spouse and will experience depressive symptoms for a longer duration than men (Fiske et al., 2009).

Life stressors are defined as occurrences that have a high likelihood to cause personal stress and require persons to change their normal activities (Gitterman, 2011) such as the death of a spouse or being diagnosed with an illness. Two approaches to studying life stressors have been to study the (1) impact of an individual life stressor or the (2) accumulation of life stressors (Gitterman, 2011). As life stressors happen at a greater frequency in older adulthood (Friske, 2009; Kraaij \& de Wilde, 2001), the number of life stressors that older adults experience has a strong influence on the development of depression (Kraaij \& de Wilde, 2001; Nolen-Hoeksema \& Ahrens, 
2002), particularly for older females (Kessler, 2003; Nolen-Hoeksema et al., 1999). If left unattended, risk factors such as life stressors are cumulative and can be difficult to treat over time (Gitterman, 2011). Overall, both the life span perspective and the risk and resiliency perspective address biological, sociocultural, and individual processes in human development and by considering the person in the environment, it allows for a comprehensive examination of depression in older females including the interacting effects and cumulative impact of life stressors.

Risk and resilience perspective. A risk-focused approach suggests that problems can be prevented or at least reduced by identifying the factors that increase the risk of having these problems and then eliminating them or reducing their effects (Hawkins, Catalano, \& Miller, 1992). Risk factors may increase the likelihood that a person is affected by a mental illness (Institute of Medicine, 1994). Risk factors are characteristics such as age, gender, family history, or lack of social support that make it more likely that a person will develop a problem such as depression (Garmezy, 1991;Werner \& Smith, 1992).

The concept of risk originated in the medical field in the 1980s, in the public health field of epidemiology (Bush et al., 1989; Vartiainen et al., 1998). Since the 1980s, the definition of a risk factor has changed from a fixed, specific circumstance such as age or gender to a more general circumstance that is modifiable and/or related to life span (Avison, 1996). For example, risk factors may change with the person's life span. 
Specific risk factors may have more weight than a single risk factor. One risk factor may not have a substantial effect compared to multiple risk factors in interaction with each other (Friske et al., 2009). For example, a single risk factor may not lead to depression; rather, multiple risk factors in interaction with other risk factors may lead to depression. Risk factors such as life stressors are cumulative and can be difficult to treat over time (Gitterman, 2011) and can make depression complex and difficult to treat.

When using the risk-focused approach multiple risk factors in combination need to be assessed and put into the perspective of development. For example, adults may have different risk factors for depression and these risk factors may change with the person's developmental course; therefore, risk factors in early life stages may not be considered risk factors in older adulthood.

While looking at risk factors, protective factors have an important role in mediating the effects of risk factors (O'Connell, Boat, Warner, 2009; Rutter, 1987; Saleebey, 1996). Resilience theory focuses on persons' strengths and resources and is often differentiated from the risk-focused approach (Rutter, 1987; Saleebey, 1996). Resilience theory recognizes the importance of protective factors as mediating the effects of risk factors (Rutter, 1987; Saleebey, 1996). Protective factors are defined as factors that change or enhance a person's response to a problem and reduce the negative impact of a risk factor on a problem outcome (Rutter, 1979; Rutter, 1985; Scambler, 2008). Protective factors can be physiological, psychosocial, geographical, cultural, and economical (Scambler, 2008; Hawkins, Catalano, \& Miller, 1992; Kraemer et al., 1997). An integrated life span risk and resilience perspective. A life span risk and resilience perspective is based on the idea that depression throughout the life span is 
multi-determined, meaning it is the result of many causes, including biological, psychological, or social causes. For example, some people have a genetic predisposition to depression, yet family and environmental conditions may lead to the development of depression. A life span risk and resilience perspective takes into account the whole person and does not rely on the symptom-based approach to identifying and treating depression; rather, a life span risk and resilience perspective allows for a comprehensive examination of (a) the influence of contextual factors associated with development of depression, (b) the cumulative effects of risk factors on the development of depression; and (c) the psychopathy of depression related to gender.

\section{Rationale for Study}

There were two important purposes for conducting this study including clinical and coordinated cross-systems treatment implications. Applying the integrated model of life span perspective (Baltes, 1987; Baltes, Lindenberger, \& Staudinger, 2006) and the risk and resilience perspective (Hawkins, Catalano, \& Miller, 1992) to examine factors that predict gender-based differences in depression treatment outcomes in older primary care patients and how gender effects depression treatment outcomes in older primary care patients. This study aimed (a) to bring attention to gender-based differences in predictors of depression and depression treatment outcomes based on longitudinal analyses and has the potential to inform the development of gender-based depression screening instruments for older primary care patients and early interventions for primary care settings; and (b) to inform coordinated cross-systems treatment of depression in older females in primary care settings. 
First, it is important that older females with depression are being treated for depression so that they live quality, self-directed lives. Everyone deserves treatment and the opportunity to live a fulfilling life no matter what age; however, older adults with depression commonly receive inadequate screening (O'Connor, Whitlock, Gaynes, \& Beil, 2009) that may result in misdiagnoses, no diagnoses, or even death. Providers may just treat the symptoms and not the factors that are cumulative and contributing to underlying matters. The intention of this secondary data analysis was to further the current understanding of the factors that are the strongest predictors of depression in primary care patients and inform the development of gender-based screening instruments for depression, early preventative interventions efforts, and advance existing evidencebased practice interventions in primary care setting.

Second, this study has important implications for coordinated cross-systems treatment of depression in older females in primary care settings. There are two common systems of care in the primary care setting: referral and integrated care. Many patients do not receive integrated care treatment; rather patients receive depression medication from their primary care physician or a referral for psychotherapy as the standard of care for depression treatment (Robinson et al., 2005), which encompasses sequential or parallel treatment (Mueser Noordsy, Drake, \& Fox, 2003). Sequential treatment provides treatment to patients for either a physical health, substance abuse, or mental health disorder, but they are not treated for these problems simultaneously; rather, once one of the disorders has stabilized, then providers treat the other co-occurring disorder (Burnam \& Watkins, 2006; Mueser, Noordsy, Drake, \& Fox, 2003). Although this is the standard 
of care for depression, this treatment approach makes it difficult to identify which disorder is the primary problem and fails to recognize that each disorder may exacerbate the other disorder (Mueser, Noordsy, Drake, \& Fox, 2003).

The parallel treatment model has different providers, who are experts in their respective fields of physical health, substance abuse, or mental health, treat the disorders simultaneously usually at different agencies (Burnam \& Watkins, 2006). However, this approach presents substantial barriers to treatment, such as treatment that may not be covered by health insurance companies, the patient may not follow through with referrals, and the services are poorly coordinated (Drake, Mercer-McFadden, Mueser et al., 1998; Havassy, Shopshire, \& Quigley, 2000; Mueser, Noordsy, Drake, \& Fox, 2003). Sequential and parallel treatment models have a small positive effect on clinical outcomes of patients (Drake et al., 1998; Havassy et al., 2000); rather, limited research suggests that integrated treatment has produced the most favorable outcomes.

Primary care settings are considered ideal locations for integrated care for mental health services for older adults. Since older adults have the greatest likelihood to turn to their primary care physicians for mental health and/or substance use services (Levkoff et al., 2004), primary care physicians can have a major role in the identification of depression. Co-locating mental health and physical health services in primary care settings for older adults is an emerging intervention that has shown to increase access and engagement in mental health and substance abuse services among older adults (Bartels et al., 2004; Gallo et al., 2004; Oslin et al., 2006). For older adults, co-located services have resulted in positive clinical outcomes (Gallo et al., 2004; Oslin et al., 2006), and 
increased communication between cross-system providers, reduced stigma, coordination of care, and increased engagement in mental health and substance abuse treatment (Gallo et al., 2004). The co-location of services is particularly helpful for older adults since accessing services is difficult for this population due to functional limitations (Webber, Porter, \& Menec, 2010) and transportation barriers (Hess, 2009). This study used a realworld setting and examined two of the most common systems of care that older females will encounter: enhanced specialty referral and integrated care. The real world nature of this data provides an opportunity to inform the development of gender-based screening and early preventative intervention efforts across both enhanced referral and integrated systems of care. Since the United State's health care reform has placed great emphasis on cross-systems care through the passing of the Patient Protection and Affordable Care Act (H. R. 3590) this study also has important implications for the examination of genderbased cross-systems treatment of depression. 


\section{CHAPTER II. LITERATURE REVIEW}

One of the major difficulties in understanding depression is the uncertainty of what constitutes depression in older adults, and older females specifically. There is no agreement about how to define depression and its precursors in older adults. Several factors need to be considered. First, late life depression affects both males and females, but the rates of depression are higher in older females compared to older males (Djernes, 2006; Kessler et al., 2003). In the general population, population-based studies have found higher lifetime and one-year prevalence rates of major depressive disorder in females compared to males (Burt \& Stein, 2002; Kessler et al., 1993; Kessler et al., 1994a; Kessler et al., 1994b; Kessler et al., 2003; Robin et al., 1991). Burt and Stein (2002) found that "females have a lifetime rate of major depressive disorder 1.7 to 2.7 times greater than men" (p. 258). For adults aged 65 years and older, depression affects between one and four percent while subsyndromal depression affects between 10 and 15\% (Blazer, 2009; Thomas \& O’Brien, 2006). Cuijpers et al. (2007) found that 20\% of community-dwelling older adults with subsyndromal depression later developed major depressive disorder.

While the most common mental health problem in adult aged 65 and older is depression (Sözeri-Varma, 2012), more than half of adult aged 65 years are diagnosed with major depressive disorder in their life late. Among mental health inpatient and outpatient consumers, $52 \%$ of adults had their first onset of major depressive disorder at age 60 or older ( $n=73 ; 68.4$ years, $S D=7.0$ years; 25 males and 48 females) (Brodaty et al., 2001) while $71 \%$ of older consumers aged 65 years and older in home health care 
reported their first episode of depression ( $N=539,351=$ females) (Bruce, 2002). Females frequently have early onset depression while males have late-onset depression (Fiske et al., 2009; Lavretsky, 1998), yet research suggests that females have prolonged and recurrent episodes of depression (Fiske, Wetherell, \& Gatz, 2009).

Second, depression rates may actually be higher than currently documented for females since underdiagnosis and misdiagnosis of depression is common in older adults (Substance Use and Mental Health Services Administration [SAMHSA], 2011). This is because older females can experience depressive symptoms, yet may not meet criteria for a diagnosis of major depressive disorder using the Diagnostic and Statistical Manual of Mental Disorders-V (DSM-V). A major depressive episode is defined by the DSM-V as the following:

A. five (or more) of the following symptoms have been present during the same 2-week period and represent a change from previous functioning; at least one of the symptoms is either (1) depressed mood or (2) loss of interest or pleasure. Note: Does not include symptoms that are clearly due to a general medical condition, or mood-incongruent delusions or hallucinations.

(1) depressed mood most of the day, nearly every day, as indicated by either subjective report (e.g., feels sad or empty) or observation made by others (e.g., appears tearful); (2) markedly diminished interest or pleasure in all, or almost all, activities most of the day, nearly every day (as indicated by either subjective account or observation made by others); (3) significant weight loss when not dieting or weight gain (e.g., a change of more than five percent of body weight in a month), or decrease or increase in appetite nearly every day; (4) insomnia or hypersomnia nearly every day; (5) psychomotor agitation or retardation nearly every day (observable by others, not merely subjective feelings of restlessness or being slowed down); (6) fatigue or loss of energy nearly every day; (7) feelings of worthlessness or excessive or inappropriate guilt (which may be delusional) nearly every day (not merely self-reproach or guilt about being sick); (8) diminished ability to think or concentrate, or indecisiveness, nearly every day (either by subjective account or as observed by others); 
(9) recurrent thoughts of death (not just fear of dying), recurrent suicidal ideation without a specific plan, or a suicide attempt or a specific plan for committing suicide. (American Psychiatric Association, 2013)

Symptoms of depression in older adults may vary, and therefore, not meet the criteria in the DSM-V since the presentation of depression changes throughout the life span (Blazer, 2003; Parker, 2000; Parker et al., 2001). Research suggests older adults may not present the standard symptoms of depression as defined by the DSM-V such as dysphoria and worthlessness/guilt (Gallo, Anthony, \& Muthen, 1994); rather, older adults may present the following clusters of symptoms, including memory complaints (Butters et al., 2004; Fiske et al., 2009), anhedonia, somatic complaints, anxiety, lack of interest in personal care (Gallo \& Rabins, 1999), sleep disturbance, tiredness, loss of interest in life, hopelessness (Christensen et al., 1999), slowed movement, and agitation (Christensen et al., 1999; Parker, 2000; Parker et al., 2001). Gender-specific differences in the presentation of depression suggest that depressed older females have more appetite problems compared to older males (Kockler \& Heun, 2002). Older adults, predominantly females, also may present depression as vegetative symptoms and cognitive dysfunction (Boswell \& Stoudemire, 1996).

Third, the presentation of depression among older adults is complex due to comorbid symptoms from physical illnesses. Late life depression is commonly associated with co-morbid physical health conditions (Katon \& Kroenke, 2007; Lacro \& Jeste, 1994; Zubenko et al., 2003). Studies suggest that depression predominantly affects older females, compared to males, with the following physical health conditions: (a) diabetes (Anderson et al., 2001; Egede, Zheng, \& Simpson, 2002); (b) cardiovascular disease (Rutledge et al., 2009), and (c) obesity (Becker et al., 2001). However, co-morbid 
conditions further complicate diagnosis. According to the DSM-V, a major depressive disorder diagnoses cannot be established if depressive symptoms are due to a physical illness (American Psychiatric Association, 2013). Therefore, depression may be underdiagnosed if depression co-occurs with a physical illness.

Fourth, prescription medications may contribute to symptoms of depression (Miller et al., 1996; Proctor et al., 2008); however, it is unclear whether there is a gender difference in prescription medications association to symptoms of depression. Studies suggest that older adults with recurrent major depressive disorder have on average 4.2 physical illnesses and took on average 2.2 different medications (Miller et al., 1996). Among older adults hospitalized for major depressive disorder ( $N=195$, mean age of 76.2; $S D=7.1$ ), older adults were taking an average of 4.1 medications for physical illness upon hospital discharge (Proctor et al., 2008); however, these studies did not report on the link between specific medications and depressive symptoms.

\section{Risk Factors for Depression in Older Females}

While short-term social risk factors such as life stressors (Glass et al., 1997; Kessler, 1997) and long-term risk factors such as biological or psychological factors are associated with depression in older adults (Blazer, 2003; Bruce, 2001; Hays et al., 1997; Kraaij et al., 2002; Magaziner et al., 1990; Robinson \& Price, 1982; Sullivan et al., 1997), certain risk factors may have more of an impact on depression than others for older females. Kessler (2003) found that risk factors that affect a person's biology such as menopause, oral contraceptives, and hormone replacement therapy in females do not significantly affect levels of depression; rather, Kessler suggested the crucial component 
to understanding the higher prevalence of depression among females compared to males may be due to social risk factors. A major social risk factor for depression is life stressors (Kraaij et al., 2002).

Life stressors in older adulthood has a significant relationship with depression (Kraaij et al., 2002), particularly for older females (Kessler, 2003). Research has suggested that females respond differently to life stressors compared to males making them more vulnerable to depression (Nolen-Hoeksema et al., 1999). The frequency and severity of life stressors may be greater in older adulthood compared to younger age cohorts since the normal aging process is associated with increased frequency of life stressors such as such as death of a spouse, family member, or friend, relocating into a nursing home, or the diagnosis of chronic illnesses. The cumulative effects of multiple life stressors have the strongest relationship with depression (Nolen-Hoeksema \& Ahrens, 2002).

According to Gitterman (2011) in social work practice, there are three defined categories of life stressors: (a) challenging life transitions and traumatic experiences; (b) environmental stresses; and (c) maladaptive interpersonal processes. For the purposes of this study, challenging life transitions and traumatic experiences were examined since difficult life transitions and traumatic life events are both key risk factors for depression (Fiske et al., 2009).

When difficult life transitions come too early or too late in the life span they are likely to be stressful (Gitterman, 2011). An example of a difficult life transition that comes too late in the life span for an older woman could be developing a chronic illness. 
Further, the abruptness of a traumatic life events such as the loss of a spouse can cause personal crisis and long lasting residue of pain, severe physical, psychological, and or social loss (Gitterman, 2011). Life stressors at any age can generate associated stressors, and when life stressors are not managed or resolved additional stressors erupt (Gitterman, 2011). The following discussion uses the life span risk and resilience perspective to situate difficult life transitions and traumatic life events life stressors developmentally for older females.

Change in living condition/residence. Change in living condition and change in residences are both identified as life stressors that can affect a person's health (HolmesRahe, 1967). In the United States, approximately, five percent of older adults live in nursing homes (Johnson \& Wiener, 2006), and it is estimated that by 2030, $10 \%$ of older adults will live in nursing homes (Feder, Komisar, \& Niefeld, 2000; Sahyoun et al., 2001). Depression is common in nursing homes, approximately $20 \%$ of nursing home residents report depressive symptoms (Jones, 2002). Older females are at particular risk of depression in nursing homes since research suggests female gender is a main risk factor for experiencing depression in nursing homes (Jones, 2002).

Change in the health of a family member. As previously stated, older adults have an increased risk for multiple chronic physical conditions compared to younger adults (Keyes, 2005); therefore, family members health status are also at an increased likelihood to change as they age. While the change in the health of a family member is a life event that could have a negative impact on a person's health (Holmes-Rahe, 1967), this event can have a kindling effect and result in an older woman becoming a family 
caregiver. The majority of caregivers in the United States are females (61\%), and the average caregiver's age is 63 years (Administration on Aging [AOA], 2004). Not surprisingly, a greater number of older females are caregivers compared to older males (AOA, 2004).

Caregiving for a person with a physical health condition or dementia is a major risk factor for depression (Livingston et al., 1996; Lutzky \& Knight, 1994; Waite et al., 2004), particularly for older females since they are more likely to be caregivers than older males (HHS, 2008). Nearly half of caregivers have depressive symptoms and nearly $33 \%$ have clinically significant depression (Waite et al., 2004).

Death of a close family member, friend, or spouse. Throughout the life span, the likelihood of losing a close family member, friend, or spouse increases as individuals maturate into older adulthood. Studies have consistently found a link between depressive symptoms and bereavement in older adults (Cole \& Dendukuri, 2003; De Beurs, Beekman, \& Geerlings, 2001; Prigerson et al., 1995). Compared to males, females have a greater likelihood of developing depression or depressive symptoms after the death of a spouse and will experience depressive symptoms for a longer duration than males (Fiske et al., 2009).

Divorce and marital separation. Divorce and marital separation are significant life stressors that may affect a person's health (Holmes-Rahe, 1967). The relationship between divorce and severity of depression is comparable for both females and males (Bierman, Fazio, \& Milkie, 2006; Hughes \& Waite, 2009). However, divorce and marital separation is important to include as a life event for older females since these are 
common risk factors for depression for both males and females (Bierman, Fazio, \& Milkie, 2006; Hughes \& Waite, 2009).

Financial strain. The loss of a spouse creates additional stress for widowed females including financial strain (Umberson, Wortman \& Kessler, 1992). Financial strain is a primary risk factor for depressive symptoms (Umberson et al., 1992). Further, financial strain can have kindling effects and may result in additional life stressors such as foreclosure of mortgage or loans, change in sleeping habits, change in living condition, or change in residence.

Personal illness. Personal illness is identified as a life event that affects a person's health status (Holmes-Rahe, 1967). Throughout the life span, older age is associated with increased personal illnesses (Keyes, 2005). Older adults have an increased risk for multiple chronic physical conditions compared to younger populations (Keyes, 2005). Approximately $80 \%$ of older adults aged $65+$ years have one chronic physical condition such as heart disease, stroke, cancer, cardiovascular, and diabetes, and $50 \%$ of older adults have at least two chronic physical condition (Centers for Disease Control and Prevention [CDC], 2010; Egede et al., 2002; Rutledge et al., 2009; Simon et al., 1995; Sullivan et al., 2002; Welch et al., 2009). Chronic physical conditions are directly correlated to depression for both males and females (Fiske et al., 2009).

Throughout the life span, females have more physical health conditions than males (Anderson et al., 2001; Egede et al., 2002; Verbrugge, 1995), suggesting they are at an increased risk for co-morbid depression and chronic illnesses. Therefore, older females may experience more cumulative life stressors as related to multiple illnesses as 
they age. Studies suggest that depression and physical health conditions and disability are more prevalent among older females compared to older males for the following conditions: (a) diabetes (Anderson et al., 2001; Egede et al., 2002); (b) cardiovascular disease (Rutledge et al., 2009); (c) obesity (Becker et al., 2001); and (d) visual impairment (Jones, Taylor, \& Broadwell, 2009).

Social isolation. Some factors that may influence social isolation include life stressors such as a son or daughter leaving home, or a change in social activities possibly due to personal illness, personal injury, or change in residence (Holmes-Rahe, 1967). Population estimates of social isolation in community-dwelling older adults ranges from 10 to $43 \%$ (Smith \& Hirdes, 2009). Social isolation is a risk factor for depression (Heikkinen \& Kauppinen, 2004). However, research has not focused on older adulthood or gender-specific differences related to depression when life stressors may lead to social isolation (Li \& Ferraro, 2005).

\section{Psychological and Biological Risk Factors for Older Females for Depression}

It is important to note that the life span risk and resilience perspective suggests that social risk factors, although they may have more of an effect on the development or severity of depression in older females, can interact with biological and psychological risk factors and have a relationship with depressive symptoms. Therefore, the following section discusses the general and gender-specific psychological and biological risk factors for depression in older adults. 
Psychological risk factors. Several psychological risk factors have shown causal relationships with depressive symptoms in older adulthood, including anxiety (Thomas \& O’Brien, 2006; Blazer, 2009; Cuijpers et al., 2007), alcohol use disorders (Schonfeld et al., 2010; Glass et al., 1995; Dupree, Broskowski, \& Schonfeld, 1984), and suicidality (CDC, 2005). Gender differences were not observed in the majority of these psychological risk factors, except for suicidality.

In 2004, older adults aged 65 years and older represented $12 \%$ of the United States population, yet accounted for approximately $16 \%$ of suicides (CDC, 2005). Suicide rates for males aged 75 years and older are the highest compared to any other age cohort (36 suicides per 100,000 persons) (CDC, 2010).

Biological risk factors. Several biological risk factors have shown causal relationships with depressive symptoms in older adulthood, including medical and psychiatric co-morbidity (Blazer, 2003; Bruce, 2001; Hays et al., 1997; Kraaij et al., 2002; Magaziner et al., 1990; Robinson \& Price, 1982; Sullivan et al., 1997) including heart conditions (Carney \& Freedland, 2003; Sullivan et al., 1997), diabetes (Li, Ford, Strine, \& Mokdad, 2008), and hip fracture (Magaziner et al., 1990). Gender differences were not observed in these biological risk factors. However, research has suggested that throughout the life span, females have more physical health conditions than males (Anderson et al., 2001; Egede et al., 2002; Verbrugge, 1995).

Life stressors can exacerbate psychological and biological risk factors for depression (Fiske et al., 2009). Since there is a reciprocal influences between the individual, the relevant others, and the social environment, risk factors for depression, whether biological, psychological, or social need to be explored to examine the whole 
person. The majority of the risk factors presented above were also included in the statistical model. Any remaining variables, due to limitations of the database were not included in this study. Despite this limitation, little research has been conducted on the variables included and examined longitudinally with a nationally representative sample of primary care patients.

\section{Research Questions and Hypotheses}

The primary aim of this study was (a) to examine gender-specific differences in the correlates of depression in older primary care patients; and (b) to examine the longitudinal effect of biopsychosocial risk factors on depression treatment outcomes in different models of behavioral health care (i.e., integrated care and enhanced referral). This study maintained a focus on how gender influences the relationship between life stressors and depression while accounting for the influence of psychological and biological risk factors for depression. The variables for the study were identified and selected from a review of the literature. Secondary data from the Primary Care Research in Substance Abuse and Mental Health for Elderly (PRISM-E) study on older primary care patients were used in this study. The research questions and hypotheses for this study are presented below.

Research Question 1: To what extent does the severity of depressive symptoms change from baseline to follow-up (three-month and six-month) across two treatment conditions (i.e., integrated care and enhanced specialty referral); after adjusting for behavioral health covariates [current at-risk drinking, current anxiety, and suicidality], 
physical health covariates [chronic medical conditions], site location, and behavioral health treatment model covariates?

Hypothesis 1: After controlling for covariates, primary care patients diagnosed with major depressive disorder at baseline will show a decline in the severity of their depressive symptoms in both treatment conditions at follow-up.

Research Question 2: To what extent is the change in the severity of depressive symptoms from baseline to follow-up (three-month and six-month) across two treatment conditions (i.e., integrated care and enhanced specialty referral) related to frequency and severity of life stressors at baseline after adjusting for covariates noted in research question 1 ?

Hypothesis 2a: Patients who experience life stressors will report greater severity of depressive symptoms at baseline among primary care patients diagnosed with major depressive disorder.

Hypothesis 2b: Patients reporting life stressors at baseline will experience less of a decline in the severity of their depressive symptoms in both treatment conditions at follow-up.

Hypothesis 2c: Patients who report more high level life stressors will experience more severe depressive symptoms at baseline. 
Hypothesis 2d: Patients reporting more high level life stressors at baseline will experience less of a decline in the severity of their depressive symptoms in both treatment conditions at follow-up (three-month and six-month).

Research Question 3: To what extent is the change in the severity of depressive symptoms from baseline to follow-up (three-month and six-month) across two treatment conditions (i.e., integrated care and enhanced specialty referral) related to patients' gender, after adjusting for covariates noted in research question 1 ?

Hypothesis 3: Males will report a greater decline in depressive symptoms than females from baseline to follow-up (three-month and six-month) across two treatment conditions (i.e., integrated care and enhanced specialty referral).

Research Question 4: To what extent is the change in the severity of depressive symptoms from baseline to follow-up (three-month and six-month) across two treatment conditions (i.e., integrated care and enhanced specialty referral) related to the interaction between gender and life stressors after adjusting for covariates noted in research question $1 ?$

Hypothesis 4a: Females with life stressors at baseline will show less of a decline in the severity of depressive symptoms at follow-up (three-month and six-month) compared males diagnosed with major depressive disorder who report experiencing life stressors at baseline. 
Hypothesis 4b: Females diagnosed with major depressive disorder or subthreshold depression with greater high level life stressors at baseline will show less of a decline in the severity of depressive symptoms at follow-up (three-month and sixmonth) compared to males diagnosed with major depressive disorder who report experiencing greater high level life stressors at baseline.

\section{Significance of Study}

Depression in older adult females is a significant and growing problem (Burt \& Stein, 2002; Fiske, Wetherell, \& Gatz, 2009). Females who experience high levels of life stressors across the life span are at higher risk for developing problems with depression than their male counterparts (Fiske et al., 2009; Kessler, 2003; Nolen-Hoeksema et al., 1999). Research has focused primarily on reducing the symptoms of depression for the general aging population; however, this study focused on gender-specific differences in the correlates of depression. The medical model characterizes depression using the symptoms-based approach while ignoring an integrated approach that examines the foundational causes of depression in their development and the risk factors they have been exposed to in their life span. In social work, human behavior is the result of the interactions between an individual and their environment (Wormer, 2007), therefore, to move away from the symptoms-based approach to depression, there is a need to examine the whole person and the underlying causes of depression, not solely treat symptoms. The intention of this study was to bring attention to an integrated approach towards understanding depression in older females for clinical social work practice, and fill the gap in knowledge on gender-specific differences in depression. 
This study also has implications for the design and timing of interventions in primary care settings. Specifically, this study aimed to develop the foundational knowledge needed to develop gender-based depression screening and early preventative interventions. The need for gender-based screening and early preventative interventions is not only a significant issue for the field of social work, but also the medical field. With the increasing number of adults reaching aged 65 years and older, especially females (Vincent \& Velkoff, 2010), social workers and health care professionals in various health care settings including primary care, mental health, substance abuse, corrections and rehabilitation, assisted living facilities, and nursing homes are likely to work with older females who have depression. The study was designed to assist researchers in the development of gender-based depression screening and early preventative interventions through examining (a) the influence of contextual factors associated with depression; (b) the effects of risk factors on depression; and (c) the psychopathy of depression related to gender. Examining these contextual factors was purposeful and is intended to be used to make the designs of gender-based screening and early preventative interventions comprehensive.

This study also had important implications for health care reform policy. The United States health care reform has placed great emphasis on cross-systems care through the passing of the Patient Protection and Affordable Care Act (H. R. 3590). The study used a real-world setting and examined cross-systems of care in comparison to the standard model of care. This can provide information on the clinical effectiveness of both models and inform future health care reform policy development. 


\section{CHAPTER III. METHOD}

\section{Research Design}

This study used a longitudinal research design using secondary data from the Primary Care Research in Substance Abuse and Mental Health for Elderly (PRISM-E) study from the 1998-2004. The PRISM-E study was specifically chosen for this secondary data analysis because in contrast to the majority of studies that have focused on major or minor depressive disorder (Katon et al., 1995; Weisner et al., 2001), the PRISM-E study focused on the continuum of mental health disorders that present among older adults in primary care settings including major depressive disorder, minor depressive disorder, depression non-otherwise specified (NOS), dysthymia, anxiety, and alcohol use disorders. PRISM-E was a joint research study financially supported by the Substance Abuse and Mental Health Services Administration, the Department of Veterans Affairs, the Health Resources Services Administration, and the Centers for Medicare and Medicaid Services.

Data were analyzed that was collected during the baseline assessment, threemonth follow-up, and six-month follow-up, which was collected over the telephone or inperson at one of five primary care settings. The following variables were examined: (a) participant age, (b) participant race, (c) participant gender, (d) participant education level, (e) participant financial situation level, (f) participant marital status, (g) current major depressive disorder diagnosis, (h) current depression NOS, (i) current dysthymia diagnosis, (j) current minor depressive disorder, (k) current generalized anxiety disorder, 
(1) current panic disorder, (m) current anxiety NOS, (n) current at-risk drinking, (o)

chronic medical condition, (p) current suicidal ideation, (q) current death ideation, and (r) life stressors including loss of family/friends, lack of activities/work, family issues, money issues, medical illness, and moving/relocation.

\section{Primary Care Research in Substance Abuse and Mental Health for Elderly (PRISM-E) Design}

The data source for this study was the Primary Care Research in Substance Abuse and Mental Health for Elderly (PRISM-E) study (Levkoff et al., 2004). PRISM-E was a national multi-site study, $N=2,022$, designed to understand mental health and substance abuse service delivery models for older adults in primary care clinics (Levkoff et al., 2004). The PRISM-E study targeted a clinical sample of adults aged 65 years and older in primary care settings. Demographic information was also collected, including marital status, educational level, race and other characteristics. Additional questions collected data on specific medications used, physical health comorbidities, health care service utilization, perceived stigma, and attitudes towards mental health and substance abuse issues. Data were collected at baseline, three-month, and six-month, and 12-months and prepared by the University of Pennsylvania. Due to limitations in the 12-months data, baseline, three-month, and six-month data were used for this study. The PRISM-E study methodology was developed over the course of two years by a collaborative effort by a team of psychiatrists, primary care clinicians, statisticians, social scientists, consumers, and a federal representative (Levkoff et al., 2004). 
Sampling strategy. PRISM-E consists of a multi-site, probability sample to capture responses from participants in primary care settings. Between 1998 and 2004, adults aged 65 years and older who had an appointment at a participating primary care settings were eligible to participate in the PRISM-E study. Participating primary care settings were chosen to represent the diversity of primary care settings and patients' demographics (Levkoff et al., 2004). The study sites included five Veteran Affairs (VA) Medical Centers (Madison, Wisconsin; Philadelphia, Pennsylvania; Chicago, IL; Miami, FL; White River Junction, VT), three community mental health centers (New York, NY [2] and San Francisco, CA), and two hospital networks (Rochester, NY; Philadelphia, PA) (Levkoff et al., 2004) (see Table 3.1). For the purposes of this study, all Veteran Affairs sites were excluded since services at VA sites are distinctly different than services for the general population (see Table 3.2). Informed consent procedures were developed based on local and federal Institutional Review Board regulations (Levkoff et al., 2004). All sites were approved by their affiliated Institutional Review Board.

Initial screening. During the initial screening, trained research assistants screened eligible participants either in-person or communicated over the telephone. However, at one participating primary care site, self-report surveys were mailed to eligible participants two weeks before their scheduled primary care visit (Levkoff et al., 2004). The initial screening took an average of 15 minutes and included the 12 -item General Health Questionnaire (GHQ-12; Goldberg \& Williams, 1988; see Appendix A) to evaluate mental distress. The GHQ-12 was followed up by two questions to evaluate the participants' suicidality (Spitzer et al., 1994), and three questions about their average 
alcohol consumption (Sobell, Sobell, Leo, \& Cancilla, 1988). The participants' level of cognitive impairment was evaluated with the short Orientation-Memory-Concentration Test (Katzman et al., 1983; see Appendix B).

Patients without severe cognitive impairment with high GHQ score, who had suicidal thoughts or were considered at-risk drinkers were referred for additional baseline screening. Of the 24,930 patients who participated in the initial screening, $1102(4 \%)$ were excluded from the study, including 542 patients with severe cognitive impairment, 55 patients with incomplete screens, 194 patients who did not report their age, and 311 patients with inconsistent screen procedures. Of the 6,430 potential participants who screened positive for mental health and alcohol misuse problems, 3205 potential participants declined to participate in the baseline study or were currently under mental health and/or substance abuse treatment, and therefore, not eligible to participate.

The following is a detailed description of the initial screening questions and cutoff scores for inclusion in the study.

General Health Questionnaire (GHQ-12). General Health Questionnaire (GHQ12) assesses current mental health status. Studies have shown that the GHQ-12 score that suggests mental distress varies based on the individual's location and demographic characteristics (Goldberg, Oldehinkel, \& Ormel, 1998). The PRISM-E investigators followed the recommendations of the developers of the GHQ-12, and all of the participating primary care sites piloted the GHQ-12 to determine accurate cutoff points for patients receiving services at that location (Goldberg \& Williams, 1988). The pilot test occurred at each site as they tested ten or more older adults. For the majority of sites 
(70\%), a score of three or more on the GHQ-12 was established as the cutoff point for mental distress (Levkoff et al., 2004). At one primary care site that provided services exclusively patients who identified as Chinese, a cutoff point of one was suggested (Levkoff et al., 2004). Two other primary care sites that were comprised of mainly middle class, suburban patients, a cutoff point of two was suggested (Levkoff et al., 2004).

Suicidal thoughts. Each site had a protocol for a suicidal participant. As defined by the individual sites" suicide protocol, participants who answered "yes" to one or both of the questions were immediately referred to a mental health provider. Suicidality did not result in being excluded in the PRISM-E study if other screening and assessment criteria were met.

At-risk drinking. For the PRISM-E study, at-risk drinking was defined by the investigators as consuming eight drinks per week or five drinks or more in a day twice in a three-month period (Levkoff et al., 2004). The PRISM-E investigators defined a low drinking threshold for older adults, which is consistent with the National Institute on Alcohol Abuse and Alcoholism alcohol consumption recommendations for older adults (Dawson, 2000; United States Department of Health and Human Services [U.S. DHHS], 1995). The low drinking threshold was used to maximize alcohol sensitivity since some patients may underreport the number of alcoholic beverages they consume (Dawson, 2000; U.S. DHHS, 1995). Consumers who met criteria for at-risk drinking were automatically randomized to the integrated model (Levkoff et al., 2004).

Baseline assessment. The remaining 3,205 patients were administered the baseline assessment measures, including a general questionnaire on demographic 
information, specific medications used, physical health comorbidities, health care service utilization, perceived stigma, and attitudes towards mental health and substance abuse issues, the 20-item Center for Epidemiological Studies Depression Scale (Radloff, 1977), the MINI International Neuropsychiatric Interview (Sheehan et al., 1998), 10-item Short Michigan Alcoholism Screening Test-Geriatric Version (Blow, Gillespie, Barry, Mudd, \& Hill, 1998), three questions on alcohol consumption (Sobell et al., 1988), the 21-item Beck Anxiety Inventory (Beck, Epstein, Brown, \& Steer, 1988), and the five item Paykel Suicide Questionnaire (Paykel et al., 1974).

Conducting the baseline assessment took on average one hour and was primarily conducted in-person. However, if the participant refused an in-person interview, trained research assistants conducted the interview over the telephone. Patients were considered eligible for study participation if they met diagnostic criteria for one of the following conditions, including depression, anxiety, or at-risk alcohol drinking. These conditions were evaluated hierarchically; therefore, only one of the target conditions was assigned to each patient; however, an eligible participant could have targeted conditions in more than one category (Levkoff et al., 2004). Eligible participants were required to provide written consent to be included in the study and were asked to participate in the three-month and six-month follow-up assessments.

Of the 3,205 patients who were participants in the baseline assessment, 1,183 patients were excluded from the study, including 992 patients who did not meet criteria for target conditions, 118 patients with assessments that were not completed, and 73 patients who were diagnosed with either hypomania or psychotic disorders (Levkoff et 
al., 2004). The enrollment period for each site was nearly a year and six month or until the distinctive site enrollment goals were achieved. The final sample included 2,022 primary care patients aged 65 years or older.

For the secondary data analysis for this study, of the 2, 022 older adult primary care patients, 585 patients were included in this study. In this study at the six-month follow-up, $50.6 \%$ of the participants were randomly assigned to the integrated care model ( $n=296)$ and $49.4 \%$ of the participants were randomly assigned to the enhanced referral model $(n=289)$. One hundred percent of the total sample completed both the three-month and six-month follow-up assessments.

\section{PRISM-E Intervention}

The following is a description of the study models, including integrated care and enhanced specialty referral. To increase the generalizability of the study results, PRISME study design permitted the integrated care and enhanced specialty referral models to differ across sites. However, detailed criteria were established to standardize both models of service delivery (Levkoff et al., 2004). To be considered an integrated care model, the following criteria must be met: (a) the primary care setting must offer mental health and substance abuse services. These services were defined as assessments, treatment planning, counseling, case management, psychotherapy, and pharmacological treatment. These services must be co-located in a primary care setting with similar public sign for mental health and substance abuse services; (b) mental health and substance abuse services must be given by licensed mental health providers (i.e., social workers, case managers with mental health and/or substance abuse training, psychologists, psychiatric 
nurses, and psychiatrists); (c) there must be verbal and/or written communication concerning the patients' assessment and treatment planning between the mental health or substance abuse provider and the primary care provider; (d) the participants had an appointment scheduled with either a mental health or substance abuse provider within two to four weeks following their primary care provider visit; and (e) the treatment facility must have been functioning for six month prior to participation in the PRISM-E study (Krahn et al, 2006; Levkoff et al., 2004). Adherence to these criteria was evaluated by PRISM-E coordinating center staff during site visits (Levkoff et al., 2004).

To be considered an enhanced specialty referral model, the following criteria was met: (a) participants were referred to a specialty mental health care clinic within two to four weeks after the primary care provider visit; (b) mental health or substance abuse assessment and treatment was conducted in a different facility than the primary care provider setting by licensed mental health professionals (i.e., licensed social workers, psychologists, psychiatric nurses, psychiatrists, and case managers with mental health and/or substance abuse training); (c) if the consumer missed their first scheduled appointment, then follow-up was made with the participant to organize transportation and provide emergency consultations; and (d) the treatment facility must have been functioning for six month prior participation in the PRISM-E study (Krahn et al, 2006; Levkoff et al., 2004). Adherence to these criteria was evaluated by PRISM-E coordinating center staff during site visits (Levkoff et al., 2004).

Specific interventions were not required at each of the PRISM-E participating sites; rather, the PRISM-E investigators chose to not mandate specific clinical 
interventions because the majority of the sites had well-established treatment models inplace prior to the start of the PRISM-E study (Levkoff et al., 2004). However, participants who met criteria for at-risk drinking were automatically randomized to the integrated model. Most sites offered pharmacotherapy to treat major depressive disorder while individual therapy was used to treat minor depressive disorder in both behavioral health treatment models (see Table 3.3). Model fidelity and adherence to criteria were established through evaluations assessed by the PRISM-E Coordinating Center prior to randomization (Levkoff et al., 2004). Study sites were constantly monitored through process evaluations and site visits by the PRISM-E Coordinating Center (Levkoff et al., 2004).

\section{Data Management}

The PRISM-E data set for this study was made available by the University of Pennsylvania, Center for Mental Health Policy and Services Research. A letter of agreement between the investigator and the University of Pennsylvania, Center for Mental Health Policy and Services Research was obtained (see Appendix C). Data were encrypted and accessed through the coordinating center website in SPSS format, with separate files for codebooks, questionnaires, and other related literature. Within the SPSS data file, responses for 2, 022 participants were included. Each participant in PRISM-E data set was de-identified and assigned a unique identification number to maintain the confidentiality of the participants. 


\section{Study Sample}

Participants who completed the MINI-depression (Sheehan et al., 1998) were included in this study in order to stratify the data by depression type and accurately examine depression severity. The final sample included 585 older adult primary care patients aged 65 years and older (see Table 3.4 and Table 3.5). At the six-month followup, $50.6 \%$ of the participants were randomly assigned to the integrated care model ( $n=296)$ and $49.4 \%$ of the participants were randomly assigned to the enhanced referral model ( $n=289)$. One hundred percent of the total sample completed both the three-month and six-month follow-up assessments.

Most participants were on average aged 73.7 years $(S D=5.6$, range $65-103$ years). More than half of the sample was female $(n=378 ; 65.3 \%)$. The sample was racially and ethnically diverse, $(n=368 ; 63.2 \%)$. A third of all participants were married $(n=211$; $36.1 \%)$, and $48.1 \%$ of all participants had a high school education or more $(n=280)$. At baseline, more than half of the sample $(n=309 ; 52.7 \%)$ had major depressive disorder.

\section{Assessment Measures}

From the baseline, three-month, and six-month assessments, five measures that were used in the PRISM-E study were included in this study. The five measures included (a) a general questionnaire on demographic information, specific medications used, physical health comorbidities, health care service utilization, perceived stigma, and attitudes towards mental health and substance abuse issues; (b) 20-item Center for Epidemiological Studies Depression Scale (Radloff, 1977); (c) MINI International Neuropsychiatric Interview (Sheehan et al., 1998); (d) three questions on alcohol 
consumption (Sobell et al., 1988); and (e) the five item Paykel Suicide Questionnaire (Paykel et al., 1974).

The following is a description of the baseline measures and the psychometric properties.

General questionnaire. A general questionnaire (Appendix D) was developed to understand the characteristics of the sample. Items in the questionnaire included questions regarding age, gender, race, education (years), marital status, and financial situation. The demographic variables were coded numerically using SPSS statistical software. The demographic variables have a central role in identifying the static risk factors for co-morbid depression and at-risk drinking in older adults in order to target social work interventions and resources. The general questionnaire included questions on life stressors that cause their mental illness and medical comorbidity.

Age. Participants answered questions about their age as part of the survey, "what is your age" and "what is your birthdate". These continuous responses were included in the descriptive statistics analyses.

Gender (moderator variable and fixed effect). Participants answered questions about their gender as part of the survey, identifying as either "male" or "female". These dichotomous responses were included in the analyses.

Race. Participants answered questions about their race as part of the survey, identifying as "white”, "black", “Asian”, "Hispanic”, "Native American”, "other” or 
"refused". These categorical responses were included in the descriptive statistics analyses.

Education. Participants answered questions about their education as part of the survey, "level of school completed". These continuous responses were included in the descriptive statistics analyses. Education was dummy-coded with three categories: less than high school, high school, and any post-high school education.

Marital status. Participants answered questions about their marital status as part of the survey, identifying as "married", "separated", "divorced", "widowed", "never married", or "don't know". These nominal responses were included in the descriptive statistics analyses.

Finances. Participants answered questions about their financial situation as part of the survey, identifying as "can't make ends meet", "just enough to get along", "are comfortable" or "don't know". These categorical responses were included in the analyses.

Chronic medical conditions (covariate and fixed effect). Participants were asked a series of "yes" and "no" questions related to a medical diagnosis. Sample questions include "have you been diagnosed with cancer" and "have you been diagnosed with diabetes?" (see the Medical History Checklist in Appendix E). Participants had the opportunity to fill in their medical diagnoses as well. Participants who responded "yes" to any of the following diagnoses (a) diabetes or sugar sickness; (b) high blood pressure or hypertension; (c) heart trouble; or (d) broken hip were included in the analysis. 
Site location (covariate and fixed effect). The PRISM-E study used a multi-site research design. The general questionnaire reports which sites the patient received initial treatment from. Since interventions at each site were non-equivalent and this study did not focus on looking at outcomes based on sites, site variation was controlled by dummy coding site location and defined as a fixed effect.

\section{Behavioral health treatment model (covariate and fixed effect). The PRISM-E}

study used a multi-site research design and included two models of behavioral health care-enhanced specialty referral and integrated care. The general questionnaire reports which treatment model the patient received initial treatment from. The two treatment models were entered as a covariate and fixed effect in the statistical model.

Time. To assess changes from baseline, three-month, and six-month, a variable for time was created. The time variable was created through restructuring all the covariates over baseline, three-month, and six-month.

Life stressors (independent variable and fixed effect). Participants were shown flash cards about the causes of their mental health problem as part of the survey and were prompted to answer "yes" or "no" to the following stresses that they perceive caused their depression, including "loss of family/friends", "lack of activities/work", "family issues", "money issues", "medical illness", and "moving/relocation". The participants' dichotomous responses were included in the analysis. The life stressors measure has not been tested psychometrically. However, the PRISM-E investigators were experts in 
substance abuse and mental health for older adults and this designed this measure based in their expertise.

Since the weight of a life stressor may be different based on the participant, it was proposed that life stressors will be analyzed by individual life stressors and also analyzed and rated by the level of stress (i.e., high-level, medium-level, low-level) based on the Social Readjustment Rating Scale (Holmes-Rahe, 1967) (see Appendix F); however, it is important to note that multicollinearity findings suggested analyzing life stressors by severity would not produce reliable results with this data. Therefore, life stressors were only analyzed by individual life stressor, not severity. This is presented and discussed in detail in chapter four.

While a measure of life stressors does not exist for older adulthood, the Social Readjustment Rating Scale (Holmes-Rahe, 1967) was identified as the standard measurement of life stressors within the general population. The Social Readjustment Rating Scale (Holmes-Rahe, 1967) was developed to determine whether life stressors were associated with illnesses. This scale has been found to be a consistently reliable and valid measure (Komaroff et al., 1968; Rahe et al., 1970; Rahe et al., 1972; Woon et al., 1971).

The Social Readjustment Rating Scale (Holmes-Rahe, 1967) presents 43 life stressors and respondents mark off each life stressor that has happened to them in the last year (Holmes-Rahe, 1967). Each life stressor has an individual corresponding number that upon completion are added and the cumulative score gives an estimate of how life stressors affect health. A person with a score of 300 or higher is at-risk for illness, while a 
score of 150-299 suggests the risk for illness is moderate, while a score of less than 150 suggests a person has a small risk of illness (Holmes-Rahe, 1967).

For the purposes of this study, life stressors was defined categorically and scored based on level of severity as defined by the Social Readjustment Rating Scale (HolmesRahe, 1967) using a condensed scoring formula. If a participant has experienced "loss of family/friends" this is worth six points, followed by "family issues" equal to five points, "medical illness" equal to four points, "lack of activities/work" equal to three points, "money issues" equal to two points, and "moving/relocation" equal to one point. A score of 21-15 means high-level of life stressors, a score of 14-7 is medium-level of life stressors, and a score of 6 or lower is low-level of life stressors. Again, it is important to note that multicollinearity findings suggested analyzing life stressors by severity would not produce reliable results with this data. Therefore, life stressors were only analyzed by individual life stressor, not severity. This is presented and discussed in detail in chapter four.

At-risk drinking. The alcohol consumption questions (Sobell, Sobell, Leo, \& Cancilla, 1988) identified at-risk drinkers. Participants were asked three questions related to their drinking behavior. Sample questions include "have you had a drink of alcohol current?" and "how many alcohol drinks have you had in the past week?". The participants' dichotomous and continuous responses were scored and included in the analysis.

At-risk drinking (covariate and fixed effect). While consumption and problembased measures assess the severity of an alcohol use disorder, measuring at-risk drinking may assist in preventing alcohol-related problems. Identifying at-risk drinking is a goal 
for early preventative interventions for alcohol-related problems and disability (Oslin et al., 2006; U.S. Preventive Services Task Force, 2004). At-risk drinking was defined as consuming eight drinks per week or five drinks or more in a day twice in a three-month period (Levkoff et al., 2004). The PRISM-E investigators established a low drinking threshold, which is aligned with the National Institute on Alcohol Abuse and Alcoholism guidelines for moderate drinking for older adults (Dawson, 2000; U.S. DHHS, 1995). Atrisk drinking was classified using three questions about alcohol consumption (Sobell et al., 1988; see Appendix G).

\section{Center for Epidemiological Studies Depression Scale. The Center for} Epidemiological Studies-Depression (CES-D; Radloff, 1977; see Appendix H) is a 20item interviewer-administered scale that measures depressive symptomology in the past week. For each question, the participants' response ranges from 0 to 3 (zero = "rarely or none of the time", one = "some or little of the time", two = "moderately or much of the time", and three = "most or almost all the time") (Radloff, 1977). Cumulative scores could range from zero to 60 , higher scores suggest more substantial symptoms of depression. The CES-D has established cutoff scores of 16 or more that are indicative of determining if an individual is at-risk for clinical depression (Lewinsohn, Seeley, Roberts, \& Allen, 1997). The CES-D has good sensitivity and specificity (Lewinsohn, Seeley, Roberts, \& Allen, 1997) and high internal consistency ranging from .85 to .90 using Cronbach's alpha coefficients (Radloff, 1977). The CES-D is a reliable measure for assessing depression symptomology across classifications of race, gender, and age (Knight, Williams, McGee \& Olaman, 1997; Radloff, 1977; Roberts, Vernon, \& 
Rhoades, 1989). The CES-D is a good measure for depression symptomology, however, research has suggested the CES-D may not be an adequate instrument to use for screening for clinical depression or major depressive disorder (Roberts, Vernon, \& Rhoades, 1989).

Depression severity (dependent variable). Since older adults do not necessarily meet criteria for a diagnosis of major depressive disorder using the standard diagnostic instrument, and empirical research on geriatric depression has used classifications such as minor depressive disorder, subsyndromal depression, dysthymia, or clinically relevant depression, depression was defined as major depressive disorder and other types of depression that are not as severe including dysthymia. Severity of depression was classified using the CES-D (higher scores reflect higher severity) and was used to examine the changes in participants' levels of depression.

MINI International Neuropsychiatric Interview. The Mini-International Neuropsychiatric Interview (MINI; Sheehan et al., 1998; see Appendix J) is a brief structured diagnostic interview that was designed by psychiatrists and mental health providers in both the United States and Europe (Sheehan et al., 1998). The MINI uses diagnostic criteria from the Diagnostic and Statistical Manual of Mental Disorders (DSM-IV) and International Statistical Classification of Diseases and Related Health Problems (ICD-10) (Sheehan et al., 1998). The structured diagnostic interview is approximately 15 minutes and was designed for both epidemiological and multisite research studies. The MINI is comprised of multiple modules including major 
depressive disorder (current and lifetime), dysthymia (current), panic disorder (current and lifetime), agoraphobia (current and lifetime), social phobia (current and lifetime), simple phobia (current and lifetime), generalized anxiety disorder (current), obsessive compulsive disorder (current), psychotic disorder (current and lifetime), alcohol abuse (current), alcohol dependence (current), drug abuse (current), drug dependence (lifetime), anorexia (lifetime), bulimia nervosa (lifetime), and post-traumatic stress disorder (lifetime) (Sheehan et al., 1998). For each disorder, participants are asked a series of "yes" and "no" questions. The MINI does not examine symptom severity, disability, or medically explained symptoms (Sheehan et al., 1998).

Lecrubier et al. (1997) examined the psychometric properties of the MINI. Threehundred and forty-six patients (296 participants had mental health disorders and 50 participants did not had mental health disorders) were administered the MINI and the Composite International Diagnostic Interview (CIDI). The interviewers in this study were trained to use both the MINI and the CIDI. The MINI interview lasted approximately 21 minutes and, and the CIDI interview lasted 92 minutes. Kappa coefficient, sensitivity, and specificity found to be good or very good for all diagnoses, except for generalized anxiety disorder $(\mathrm{kappa}=0.36)$, agoraphobia $($ sensitivity $=0.59)$ and bulimia $($ kappa $=0.53)($ Lecrubier et al., 1997). Overall, the inter-rater and test-retest reliability were considered good (Lecrubier et al., 1997). The MINI has been used repeatedly with individuals aged 65 years and older (Godin, Elbejjani, \& Kaufman, 2012). 
Major depressive episode (MDE) module. This measure includes 11-items associated with the DSM-IV diagnosis of major depressive episode. The patient-rated version of the MINI- MDE is valid and reliable when assessed in contrast to the patientversion of the Structured Clinical Interview for Diagnostic and Statistical Manual of Mental Disorders III (SCID-P). Specifically, agreement between both instruments was good for major depressive disorder (kappa $=0.55$, sensitivity of .77 , and specificity of .79) (Sheehan et al., 1998). Inter-rater and test-retest reliability were excellent, 1.00 and .87 respectively (Sheehan et al., 1998). The ages of participants in this study ranged from 18-86 years $(M=44.8, S D=15.1)$ years (Sheehan et al., 1998).

Major depressive episode (current; covariate and fixed effect). A trained research assistant asked the participants "yes" or "no" questions about depression symptoms as part of the baseline assessment. Sample questions include "have you been consistently depressed or down, most of the day, nearly every day, for the past two weeks?" and "in the past two weeks, have you been much less interested in most things or much less able to enjoy the things you used to enjoy most of the time" (Sheehan et al., 1998).

If the participant answered "yes" to five or more of the above answers, then the participant had a current major depressive episode and were eligible to be asked additional questions related to recurrent major depressive episode. Participants with current major depressive episode were then asked "during your lifetime, did you have other episodes of two weeks or more when you felt depressed or uninterested in most things, and had most of the problems we just talked about?" and "in between 2 episodes of depression, did you ever have an interval of at least 2 months, without any depression 
and any loss of interest?" (Sheehan et al., 1998). If the participant answered "yes" to both questions, then the participant had a recurrent major depressive episode. Participants' dichotomous responses and associated score were aggregated and included in the analyses in the descriptive statistics and also the statistical model. Since the dependent variable measures depression severity it was imperative to stratify data by depression diagnosis using the MINI-depression (Sheehan et al., 1998).

Minor depressive disorder (current; covariate and fixed effect). Participants were diagnosed with minor depressive disorder if the participant did not meet criteria for major depressive disorder on the MINI, but reported at least two depressive symptoms over the past four weeks. Participants' dichotomous responses and associated score were aggregated and included in the analyses in the descriptive statistics and also the statistical model. Since minor depressive disorder, depression NOS, and dysthymia are qualitative similar, these three variables were grouped together and defined as subthreshold depression. Again, since the dependent variable measures depression severity it was imperative to stratify data by depression diagnosis using the MINI-depression (Sheehan et al., 1998).

Depression NOS (current; covariate and fixed effect). Participants were diagnosed with depression NOS if the participant did not meet criteria for major depressive disorder or minor depressive disorder on the MINI, but reported at least two depressive symptoms over the past two weeks. Participants' dichotomous responses and associated score were aggregated and included in the analyses in the descriptive statistics and also the statistical model. Since minor depressive disorder, depression NOS, and dysthymia are qualitative 
similar, these three variables were grouped together and defined as part of subthreshold depression.

Dysthymia module. This measure includes 9-items associated with the Diagnostic and Statistical Manual of Mental Disorders-Fourth Edition (DSM-IV) diagnosis of dysthymia. Overall, the agreement between the MINI Dysthymia and the SCID-P was good ( $\kappa$ of 0.11 , sensitivity of .17, and specificity of .98 (Sheehan et al., 1998).

Dysthymia (current; covariate and fixed effect). If the participant met criteria for major depressive disorder, they were asked questions related to dysthymia. Sample questions include (a) "have you felt sad, low or depressed most of the time for the last two years?", (b) “was this period interrupted by your feeling OK for two months or more?", (c) "during this period of feeling depressed most of the time: 'did your appetite change significantly?', 'did you have trouble sleeping or sleep excessively?', ‘did you feel tired or without energy?', ‘did you lose your self-confidence?', 'did you have trouble concentrating or making decisions?', 'did you feel hopeless?'. If the participant answered "yes" to question one and two, and responded "yes" to two of the questions in number three, then the participant had a diagnosis of dysthymia. Participants' dichotomous responses and associated score were aggregated and included in the analyses. Since minor depressive disorder, depression NOS, and dysthymia are qualitative similar, these three variables were grouped together and defined as part of subthreshold depression.

Generalized anxiety disorder module. This measure includes 10-items associated with the Diagnostic and Statistical Manual of Mental Disorders-Fourth Edition (DSMIV) diagnosis of generalized anxiety disorder. Overall, the agreement between the MINI 
generalized anxiety disorder and the SCID-P was good ( $\kappa$ of 0.45 , sensitivity of .80, and specificity of .73 (Sheehan et al., 1998).

Generalized anxiety disorder (covariate and fixed effect). Sample questions include (1) "Have you worried excessively or been anxious about several things over the past 6 months?", (2) "Do you find it difficult to control the worries or do they interfere with your ability to focus on what you are doing?", (3) "When you were anxious over the past 6 months, did you, most of the time: (a) Feel restless, keyed up or on edge?; (b) feel tense?" If the participant answered "yes" to three or more questions in question three, then the participant had a diagnosis of generalized anxiety disorder. Participants' dichotomous responses and associated score were aggregated and included in the analyses.

Anxiety disorder NOS (covariate and fixed effect). Participants were diagnosed with anxiety disorder NOS if the participant did not meet criteria for generalized anxiety disorder on the MINI, but responded "yes" to (a) "Have you worried excessively or been anxious about several things over the past 6 months?" and (b) "Do you find it difficult to control the worries or do they interfere with your ability to focus on what you are doing?". Participants' dichotomous responses and associated score were aggregated and included in the analyses in the descriptive statistics and also the statistical model.

Panic disorder module (current). This measure includes 18 -items associated with the Diagnostic and Statistical Manual of Mental Disorders-Fourth Edition (DSM-IV) diagnosis of panic disorder. Overall, the agreement between the MINI generalized 
anxiety disorder and the SCID-P was good ( $\kappa$ of 0.76 , sensitivity of .84 , and specificity of .93 (Sheehan et al., 1998).

Panic disorder (covariate and fixed effect). Sample questions include (1) "Have you, on more than one occasion, had spells or attacks when you suddenly felt anxious, frightened, uncomfortable or uneasy, even in situations where most people would not feel that way? Did the spells peak within 10 minutes?", (2) "At any time in the past, did any of those spells or attacks come on unexpectedly or spontaneously, or occur in an unpredictable or unprovoked manner?", (3) "Have you ever had one such attack followed by a month or more of persistent fear of having another attack, or worries about the consequences of the attack?", and (4) "During the worst spell that you can remember: (a) did you have skipping, racing or pounding of your heart? (b) did you have sweating or clammy hands? (c) were you trembling or shaking? (d) did you have shortness of breath or difficulty breathing? (e) did you have a choking sensation or a lump in your throat? (f) did you have chest pain, pressure or discomfort? (g) did you have nausea, stomach problems or sudden diarrhea? (h) did you feel dizzy, unsteady, lightheaded or faint? (i) did things around you feel strange, unreal, detached or unfamiliar, or did you feel outside of or detached from, part or all of your body? (j) did you fear that you were losing control or going crazy? (k) did you fear that you were dying? (1) did you have tingling or numbness in parts of your body? (m) did you have hot flashes or chills?" If the participant answered "yes" to four or more questions in question four, then the participant had a diagnosis of panic disorder. Participants' dichotomous responses and associated score were aggregated and included in the analyses. 
Paykel Suicide Questionnaire. The Paykel Suicide Questionnaire (Paykel et al., 1974; see Appendix I) was used to determine suicidal ideation and death ideation. The Paykel Suicide Questionnaire consists of five questions about suicidal ideation or behavior in the past 12 months. The Paykel suicide scale is a five-item interview that asks "yes" and "no" questions. Paykel suicide scale is useful for screening; however, the reliability and validity of this measure need more research (Hunsley \& Mash, 2008).

Suicidality (current; covariate and fixed effect). Participants answered questions about suicidal behavior, attempts, and thoughts as part of the baseline assessment. Paykel suicide questions included three mutually exclusive groups: (1) no ideation (no Paykel items endorsed); (2) death ideation, answering yes to question one and/or two, "has there been a time in the last year when you felt life was not worth living?" or "has there been a time in the last year that you wished you were dead, for instance that you would go to sleep and not wake up?"; and (3) suicidal ideation, answering yes to question three, four, and/or five, "has there been a time in the last year that you thought of taking your own life, even if you would not really do it?", "has there been a time in the last year when you reached the point where you seriously considered taking your own life, or perhaps made plans how you would go about doing it?", and "in the last year, have you made an attempt on your life?". These dichotomous responses for suicidal ideation and death ideation were calculated, scored according to Paykel Suicide Questionnaire (Paykel et al., 1974), and then included in the analyses.

\section{Protection of Human Participants}

There were no foreseeable risks in this study project those typically associated with this specific type of research. All necessary precautions to protect the health, safety, 
and the legal rights of all clients were taken. This included abiding by all federal and local statutes and ethical principles related to provision of mental health, substance abuse, and medical services. An IRB application was submitted to Florida International University’s Institutional Review Board and approved on November 25, 2013. The primary investigator of this study is IRB Human Subjects Research certified (Appendix $\mathrm{K})$.

Privacy and confidentiality. In addition to the current policies and procedures that govern the behavior of staff and access to information, the data collected in this secondary data analysis did not contain any identifying information (see Appendix $\mathrm{C}$ for letter of agreement). All data were de-identified and only the Coordinating Center had the coding matrix that links clients with their identification number. Nonetheless the confidentiality of data collected from all sources were safeguarded through coding mechanisms. The names of research subjects have been omitted from the data forms and a numerical code has been assigned. Any publications from this study will not include any identifying information.

Integrity and privacy of the data was achieved using industry-standard encryption and a granular security permission scheme down to information at the individual patient level. Redundant server hardware deployment and database backups ensured data safety. Several levels of password protection were implemented. The primary investigator changed passwords regularly. After data entry and verification, all original data were stored in locked file cabinets, which only was accessible to the primary investigator. 
Fair selection of participants. The sample was multi-ethnic, made up $63.2 \%, n=368$, individuals identified as an ethnic minority The participants were selected to ensure that the subjects were representative of the United States in terms of the distribution of minority individuals. The mean age of the sample was 73.7 years $(S D=5.6)$. Females constituted $65.3 \%$ of the sample.

Risk/benefit. Based on the primary investigator's experience with conducting secondary data analyses, there was minimal risk and no cost to the subjects. There were no invasive medical procedures or use of administrative data. When the proper precautions were taken, and the lack of considerable risks associated with study procedures were weighted against the potential benefits, to both individual and society, the benefits clearly outweighed the risks of study participation.

\section{Data Analysis}

Quantitative data were analyzed using SPSS 19 statistical software (IBM SPSS Statistics, 2010) (a) to examine gender-specific differences in the correlates of depression in older primary care patients; and (b) to examine the longitudinal effect of biopsychosocial risk factors on depression treatment outcomes in different models of behavioral health care (i.e., integrated care and enhanced referral).

Descriptive statistics. The demographic data were analyzed using SPSS version 19 (IBM SPSS Statistics, 2010). For the preliminary analyses, assumptions of normality were examined by each site. First, homogeneity of variance was considered and data were checked for univariate and multivariate normality through screening for outliners 
and skewness and kurtosis estimates. Second, descriptive statistics and bivariate relationships of the variables by treatment group were assessed for multicollinearity using collinearity diagnostics. Third, based on multicollinearity findings, variables were deleted from the statistical models using the recommended correlation cut-off to screen for multicollinearity ( $r>0.70$ between predictor variables) (Tabachnick \& Fidell, 2001). It is important to note, that multicollinearity among the variables was likely present when using the "severity of life stressors" variable. Therefore, "severity of life stressors" variable was excluded from the hierarchical linear modeling analysis. The modified research questions are presented below.

\section{Hierarchical linear modeling (HLM): Linear mixed model approach. To}

control for site variation, non-equivalence of interventions, and adjust for within-person correlation over time, a linear mixed model approach was chosen as the appropriate hierarchical linear model (Garson, 2013) to assess changes in severity of depression, assuming assumptions were not violated. The following assumptions were considered: (a) normal distribution of variables; (b) level-2 cases should be independent; (c) level-1 cases should be dependent; and (d) homogeneity of variance was achieved for the variability in the level-1 cases (Woltman et al., 2012).

Hierarchical linear modeling (Bryk \& Raudenbush, 1992; Hox, 1995; Snijders \& Bosker, 1999) is a robust statistical method that can be used with repeated measures data to evaluate multi-level data (Kim, Solomon, \& Zurlo, 2009; Shin, 2009). Hierarchical linear modeling is commonly used in behavioral health fields such as nursing (Shin, 
2009), psychiatry (Cervantes, 2009), and is increasingly used in the field of social work (Kim, Solomon, \& Zurlo, 2009).

Using SPSS version 19 (IBM SPSS Statistics, 2010), four models were tested using hierarchical linear modeling (Bryk \& Raudenbush, 1992; Hox, 1995; Snijders \& Bosker, 1999). To address the research questions and adjust the within-subject variation and correlation, a random-intercept model was chosen as the appropriate model. The intercepts in these models were allowed to vary; therefore, the depression severity scores for each individual were predicted by the intercept, which varied across the groups.

The potentially confounding effect of (a) current at-risk drinking was controlled for by the baseline, three-month, and six-month values of the alcohol consumption questions (Sobell, Sobell, Leo, \& Cancilla, 1988). At-risk drinking was defined as consuming eight drinks per week or five drinks or more in a day twice in a three-month period (Levkoff et al., 2004); (b) current generalized anxiety disorder, panic disorder, and anxiety NOS were controlled for by incorporating the baseline values of the MINIAnxiety (Sheehan et al., 1998); (c) patients who screen positive for suicidal ideation and death ideation were controlled for by incorporating baseline, three-month, and six-month responses from the Paykel Suicide Questionnaire (Paykel et al., 1974); (d) patients with chronic medical conditions (i.e., diabetes or sugar sickness, high blood pressure or hypertension, heart trouble, or a broken hip) were controlled for by incorporating the aggregated baseline responses from the PRISM-E Medical History Checklist; (e) since interventions at each site were non-equivalent, site variation were controlled by dummy coding site location. Since these variables were not continuous, each covariates effect 
could only be controlled for in the statistical model and were considered fixed effects; and (f) behavioral health treatment model - integrated care and enhanced specialty referral were entered into the model as covariates by incorporating values that defined the treatment model from the general questionnaire into the statistical model.

The research question and methodology used in this study are presented below.

Research Question 1: To what extent does the severity of depressive symptoms change from baseline to follow-up (three-month and six-month) across two treatment conditions (i.e., integrated care and enhanced specialty referral); after adjusting for behavioral health covariates [current at-risk drinking, current anxiety, and suicidality], physical health covariates [chronic medical conditions], site location, and behavioral health treatment model?

Model one tested differences in severity of depression from baseline to follow-up (three-month and six-month) in both the integrated care and enhanced referral treatments. The contextual variable were defined as the site location, covariates and fixed effects were defined as behavioral health covariates [current at-risk drinking, current anxiety, and suicidality], physical health covariates [chronic medical conditions], site location, and behavioral health treatment model. The dependent variable was defined as scores on the CES-D (Radloff, 1977). Findings were stratified by depression type (i.e., major depressive disorder, subthreshold depression, and no depression). Findings were stratified by depression type because older adults may not present the standard symptoms of depression as defined by the DSM-V (Butters et al., 2004; Christensen et al., 1999; Fiske et al., 2009; Gallo, Anthony, \& Muthen, 1994; Gallo \& Rabins, 1999; Parker, 2000; 
Parker et al., 2001). Therefore, depression was examined by looking at depression on continuum in order to accurately estimate depression severity of older adults.

Research Question 2: To what extent is the change in the severity of depressive symptoms from baseline to follow-up (three-month and six-month) across two treatment conditions (i.e., integrated care and enhanced specialty referral) related to frequency of life stressors at baseline after adjusting for covariates?

Model two tested differences in severity of depression from baseline to follow-up (three-month and six-month) in both integrated care and enhanced referral treatments. The contextual variable was defined as the site location, covariates and fixed effects were defined as behavioral health covariates [current at-risk drinking, current anxiety, and suicidality], physical health covariates [chronic medical conditions], site location, and behavioral health treatment model. Life stressors variables were added as predictor variables and fixed effects. The dependent variable was defined as scores on the CES-D (Radloff, 1977). Findings were stratified by depression type (i.e., major depressive disorder, subthreshold depression, and no depression).

Research Question 3: To what extent is the change in the severity of depressive symptoms from baseline to follow-up (three-month and six-month) across two treatment conditions (i.e., integrated care and enhanced specialty referral) related to patients' gender, after adjusting for covariates?

Model three tested differences in severity of depression from baseline to followup (three-month and six-month) in both the integrated care and enhanced referral 
treatments. The contextual variable was defined as the site location, covariates and fixed effects were defined as behavioral health covariates [current at-risk drinking, current anxiety, and suicidality], physical health covariates [chronic medical conditions], site location, and behavioral health treatment model. Gender was added as predictor variables and fixed effects. The dependent variable was defined as scores on the CES-D (Radloff, 1977). Findings were stratified by depression type (i.e., major depressive disorder, subthreshold depression, and no depression).

Research Question 4: To what extent is the change in the severity of depressive symptoms from baseline to follow-up (three-month and six-month) across two treatment conditions (i.e., integrated care and enhanced specialty referral) related to the interaction between gender and life stressors after adjusting for covariates?

Model four tested differences in severity of depression from baseline to follow-up (three-month and six-month) in both the integrated care and enhanced referral treatments. The contextual variable was defined as the site location, covariates and fixed effects were defined as behavioral health covariates [current at-risk drinking, current anxiety, and suicidality], physical health covariates [chronic medical conditions], site location, and behavioral health treatment model. The life stressors interaction with gender were added as predictor variables and fixed effects. The dependent variable was defined as scores on the CES-D (Radloff, 1977). Findings were stratified by depression type (i.e., major depressive disorder, subthreshold depression, and no depression). 


\section{Maximum Likelihood Estimation}

The models were fit using maximum likelihood estimation. Since there is no single agreed upon method to estimate parameters in hierarchical linear modeling (Bryk \& Raudenbush, 1992; Hox, 1995; Snijders \& Bosker, 1999), maximum likelihood estimation (ML) was chosen because ML allows for the comparison of the statistical models across different predictor variables using Akaike Information Criterion (AIC) or Bayesian Information Criterion (BIC) (Garson, 2013). The AIC and BIC are measures used for model selection in non-nested data (Akaike, 1973). Since each of the hierarchical linear models in this study are non-nested, ML is the appropriate estimation technique.

\section{Covariance Structure Selection}

In the covariance structure selection, scaled identity was chosen as the covariance matrix for the random-intercept models. As stated above, there is one random effect (i.e., random intercept), and therefore, scaled identity was chosen as the covariance matrix (Bickel, 2007). Therefore, using scaled identity means there is constant variance (one variance in the diagonal) and no covariance since there is only one random effect (Bickel, 2007).

\section{Sample Size Considerations}

Multi-level models such as hierarchical linear models take into account both level-one and level-two sample sizes (Kim, Solomon, \& Zurlo, 2009). Statistical power for the level-one sample is based on the number of individual observations (patients, 
$N=585$ ) while power for the level-two sample is based on the number of groups (PRISME site locations, $N=5)$. However, there is little agreement on the number of individual observations or groups to achieve adequate power using hierarchical linear modeling; rather, sample size estimates range greatly from 30 groups to 50 groups (Kreft \& De Leeuw, 1998) to 100 groups (Afshartous, 1995). However, five groups were considered acceptable in longitudinal research (Maas \& Hox, 2005).

The basic hierarchical linear model procedure is not particularly affected by levelone or level-two sample sizes (Maas \& Hox, 2005); rather, only a small sample size at level-two, which is considered 50 or less groups results in biased estimates in the level-2 standard errors (Maas \& Hox, 2005; Snijders, 2005). Although this study met acceptable requirements for level-two sample size (Maas \& Hox, 2005), this study was not interested in the association between the site and expected treatment effect. In summary, there were no negative consequences to using the level-one $(N=585)$ and level-two sample $(N=5)$.

As gender differences in life expectancy has resulted in a larger proportion of females compared to males in the older age cohorts and the general aging population is also increasing dramatically in the United States (Vincent \& Velkoff, 2010), a life span risk and resilience perspective is a fundamental component of gender-specific and ageappropriate intervention design, development, and evaluation. Using the life span risk and resilience perspective framework (a) to examine gender-specific differences in the correlates of depression in older primary care patients; and (b) to examine the longitudinal effect of biopsychosocial risk factors on depression treatment outcomes in different models of behavioral health care (i.e., integrated care and enhanced referral) can 
enable researchers to identify important early intervention and prevention components that may alter developmental pathways for depression or reduce risk factors for worsening depression symptoms in older females.

\section{CHAPTER IV. RESULTS}

This study utilizing hierarchical linear modeling using a linear mixed modeling approach aimed (a) to examine gender-specific correlates of depression in older primary care patients; and (b) to examine the longitudinal effect of biopsychosocial risk factors on depression treatment outcomes in different models of behavioral health care (i.e., integrated care and enhanced referral).

As described in the previous chapter, the data analytic plan required several preliminary analyses to be conducted in order to test for the assumptions of hierarchical linear modeling, as well as descriptive analyses of demographic characteristics and other predictor variables, and missing data analysis. Next, hierarchical linear modeling using a linear mixed modeling approach was conducted in a series of four tests (Garson, 2013): (a) random intercept-only model; (b) random intercept model with level-2 predictors (i.e., frequency of life stressors); (c) random intercept model with level-2 predictors (i..e., gender); and (d) random intercept model with level-2 predictors (i.e., life stressors, gender, and interaction term of life stressors and gender to test for the moderation hypothesis). 


\section{Testing For Assumptions Of Parametric Tests}

Parametric tests based on the normal distribution assume (a) normal distribution, (b) homogeneity of variance, and (c) independent scores (Field, 2013). Since the dependent variable scores were independent, the following includes the tests for normality, tests of homogeneity of variance, and data transformations.

Tests for normality. Assessing the normality of the data prior to analysis is an important first step in statistical analysis because if this assumption is violated then the data can produce bias in the results (Tabachnick \& Fidell, 2012). The "split file" and "examine" function in SPSS (IBM SPSS Statistics, 2010) were used to produce both descriptive statistics and histograms to review data for normality. A skewness near zero and a kurtosis higher than three suggests normal distribution (Field, 2013). After reviewing the data based on these guidelines, skewness and kurtosis estimates suggested the data were normally distributed. Further, review of the normal curves placed over the histograms also suggested normal distribution.

Homogeneity of variance. Homogeneity of variance assumes the variance within each of the groups is equal (Field, 2013). The "split file" and "compare groups" functions in SPSS (IBM SPSS Statistics, 2010) were used to produce the variance ratio for the variables. Data were separated by behavioral treatment model. The variance ratio, also referred to as Hartley's F max (Pearson \& Hartley, 1954) was assessed since there were two samples. The variance between the group with the largest variance was compared to the group with the smallest variance (Field, 2013). If the variance ratio was less than two, 
the assumption of homogeneity of variance was met (Field, 2013). Variance ratio findings suggested that the assumption of homogeneity of variance was met.

\section{Data Transformations}

Outliers. The "explore” function in SPSS (IBM SPSS Statistics, 2010) was used to screen for outliers for all the variables used in the analyses. The scatterplot showed one outlier for the variable "age" of 290 years old. This variable value was changed to 90 years old. The remaining variables showed no significant outliers.

Missing data. Analytic decisions and procedures were considered regarding missing data in the database before running the analysis. First, to examine missing data, missing variable analyses were conducted using SPSS for each variable to test for item nonresponse. The patterns of missing data were displayed in graphs and frequencies and percentages were presented in tables. Graphs and tables were reviewed.

The following were the percentages of missing data for variables of interest: gender $(1.4 \%)$, race, $(0.5 \%)$, education $(0.7 \%)$, income $(2 \%)$, marital status $(0.3 \%)$, loss of family and friends $(0.2 \%)$, lack of activities $(0.2 \%)$, family issues $(0.2 \%)$, money issues $(0.2 \%)$, medical illness $(0.2 \%)$, moving/relocation $(0.2 \%)$, medical illness $(0.2 \%)$, diabetes $(1.9 \%)$, high blood pressure $(2 \%)$, heart trouble $(2.2 \%)$, broken hip $(3.8 \%)$, suicidality (0.9\%), and depression severity (14.3\%).

Second, a review of the literature on appropriate missing longitudinal data techniques when working with a sample of older adults suggested a dynamic approach to missing data. Research suggested the majority of missing data techniques are subjective 
and biased the missing values of the research participants as overly healthy (Engels \& Diehr, 2003). However, when working with older adults, missing data are likely related to deteriorating health (Andrew, Mitnitski, Kirkland, \& Rockwood, 2012; Engels \& Diehr, 2003; Fairclough, Peterson, \& Chang, 1998). Research suggested the most accurate missing longitudinal data method when working with a sample of older adults is the last and next method or the last observation carried forward (Engels \& Diehr, 2003).

Based on these recommendations, when longitudinal data on depression severity (dependent variable) and suicidality (covariate) were available before and after the missing value, the last and next method was used. However, if longitudinal data were only available before the missing value, the last observation carried forward method was used.

Since the remaining variables were collected at one point in time (baseline) and were missing only small amounts of data, dummy variable adjustment was chosen as the appropriate technique. Therefore, the analysis would use all the available data and not reduce sample size and subsequent power of the analysis.

\section{Descriptive Statistics}

Overall sample characteristics. All descriptive statistics were created using SPSS 19 statistical software (IBM SPSS Statistics, 2010). The final sample included 585 primary care patients aged 65 years and older. At the three-month follow-up, $50.6 \%$ of the participants were randomly assigned to the integrated care model $(n=296)$ and $49.4 \%$ 
of the participants were randomly assigned to the enhanced referral model $(n=289)$. One hundred percent of the total sample completed baseline and the three-month and sixmonth follow-up assessments.

Most participants were on average aged 73.7 years ( $S D=5.6$, range 65-103 years). More than half of the sample were female $(n=378 ; 65.3 \%)$. The majority of the sample identified as White (36.8\%), followed by Hispanic/Latino (28.9\%), African-American (17.1\%), and "other" (3.4\%).

A third of all participants were married $(n=211 ; 36.1 \%)$, and $48.1 \%$ of all participants had a high school education or more $(n=280)$. At baseline, more than half of the sample $(n=309 ; 52.7 \%)$ had a diagnosis of major depressive disorder.

Table 3.4 and 3.5 summarize the descriptive statistics for variables included in the models. Due to the categorical nature of the data, characteristics were summarized in terms of frequencies and percentages. Table 4.1 reports on changes in depression severity over time (i.e., baseline, three-month, and six-month) by gender and behavioral health treatment model. Since depression severity was measured on a continuous scale, severity is reported as mean scores.

\section{Hierarchical Linear Modeling (HLM): Linear Mixed Model Approach}

A three-level hierarchical model (i.e., time, subjects, and sites) assessed the extent that severity of depression symptoms changed at baseline, three-month, and six-month in both the integrated care and enhanced specialty referral treatment models. First-level units were "time", which was defined as baseline, three-month follow-up, and six-month 
follow-up. Second-level units were participants in the PRISM-E study, with participants limited to those who participated in the baseline, three-month, and six-month assessments based on the missing data approach used for this study (see missing data section). This resulted in a total of 585 participants for analysis with 296 participants in the integrated care group and 289 participants in the enhanced specialty referral group. Third-level units were the five research sites. Hierarchical linear modeling using the liner mixed model approach was conducted using SPSS 19 statistical software (IBM SPSS Statistics, 2010).

\section{Correlation Matrix and Variance-Inflation Factor (VIF) Results}

Before building the HLM model, correlations and VIFs of the predictor variables were reviewed for multicollinearity. In HLM, there should not be evidence of multicollinearity between predictor variables in the statistical models (Bickel, 2007; Tabachnick \& Fidell, 2012). The presence of multicollinearity can present bias in the estimated regression coefficients (Beta), standard error of the regression, and subsequently, bias the findings (Bickel, 2007; Tabachnick \& Fidell, 2012). To test for multicollinearity, correlations between predictor variables were reviewed. The recommended correlation cut-off to screen for multicollinearity was $r>0.70$ between predictor variables (Tabachnick \& Fidell, 2001).

Enhanced specialty referral. The correlation matrix for the enhanced specialty

referral group showed a minimum number of predictor variables were highly correlated $(r$ $>0.70$ ) with each other while none of the variables were highly correlated with the dependent variable. "Heart trouble" was highly correlated with "high blood pressure" ( $r=$. 
$783, p<.001)$ and a "broken hip" was highly correlated with "heart trouble" $(r=.723, p<$ .001) (see Table 4.2). The VIFs for these variables were less than four and the tolerance was greater than 0.2 indicating that multicollinearity among the variables was likely not present (see Table 4.3); however, since these two variables were correlated in the correlation matrix, "broken hip", "heart trouble", "high blood pressure" were examined further. It was determined that "broken hip" would be excluded because the sample that reported "broken hip" was small for each site, ranging from two to four persons. Therefore, it was determined the other variables would be more meaningful in the analyses.

The correlation matrix also shows "severity of life stressors" was highly correlated with "loss of family and friends" $(r=.658, p<.001)$, "lack of activities/work" $(r=.500$, $p<.001)$, "family issues" $(r=.621, p<.001)$, "money issues" $(r=.489, p<.001)$, "medical illness" $(r=.625, p<.001)$, and "moving" $(r=.462, p<.001)$. Although, the "severity of life stressors" variable does not meet the .70 cut-off point (Tabachnick \& Fidell, 2001), the VIFs were greater than four and the tolerance was less than 0.2 indicating that multicollinearity among the variables was likely present. Therefore, "severity of life stressors" variable will be excluded from the hierarchical linear modeling analysis in the enhanced specialty referral model. The modified research questions and hypotheses will be presented in the next section.

Integrated care. The correlation matrix for the integrated care group showed that a minimum number of predictor variables were highly correlated $(r>0.70)$ with each other while none of the variables were highly correlated with the dependent variable (see 
Table 4.4). "Broken hip" was highly correlated with "diabetes" $(r=.704, p<.001)$ and "high blood pressure" ( $r=.709, p<.001)$. The VIFs for these variables were less than four and the tolerance was greater than 0.2 , indicating that multicollinearity among the variables was likely present (see Table 4.5); however, since these two variables were correlated, "broken hip" and "diabetes" and "high blood pressure" were examined further. It was determined "broken hip" would be excluded because the sample that reported "broken hip" was small for each site, ranging from two to four persons. Therefore, "diabetes" and "high blood pressure" would be more meaningful variables in the analyses.

One variable, "loss of family and friends" was highly correlated with "severity of life stressors" $(r=.707, p<.001)$. The correlation matrix also showed "severity of life stressor" is highly correlated with "lack of activities/work" $(r=.623, p<.001)$, "family issues" ( $r=.644, p<.001)$, "money issues" $(r=.574, p<.001)$, "medical illness" $(r=$. $660, p<.001)$, and "moving" $(r=.551, p<.001)$. Also, for the "severity of life stressors" variable, the VIFs were greater than four and the tolerance was less than 0.2 indicating that multicollinearity among the variables was likely present. Therefore, "severity of life stressors" variable will be excluded from the hierarchical linear modeling analysis in the integrated care data. The modified research questions and hypotheses will be presented in the next section.

\section{Building Hierarchical Linear Models Using Linear Mixed Model Approach}

For HLM building models using the three levels, the model building started with an intercept-only model, in which none of the predictor variables were entered into the 
model while all of the level-one (time), level-two (participants), and level-three (sites) and covariates were entered. Since the research questions focus on the level-two sample, a linear mixed model approach was chosen as the appropriate hierarchical linear model (Garson, 2013). Linear mixed modeling approach to hierarchical linear modeling uses statistical models where only the intercept of the level-one variable (time) is modeled as an effect of the level-two grouping variables (participants) and other level-one, level-two or level-three covariates (sites) (Garson, 2013). Therefore, linear mixed model allows for a comparison between regression models across level-two variables while accounting for hierarchical data (Garson, 2013).

For the purposes of answering the research questions appropriately, all of the control variables were retained in each of models despite lack of statistical significance. The inclusion of the control variables throughout the statistical models adjusted for behavioral health covariates [depression type, current at-risk drinking, current anxiety, and suicidality], physical health covariates [chronic medical conditions], site location, and behavioral health treatment model. Further, it is important to note, throughout the analysis, San Francisco, California was considered an ideal reference group group to best represent the United States population since this site had the largest sample that was racially and ethnically diverse (Tabachnick \& Fidell, 2012).

The intercept-only model addressed research question 1 and examined to what extent does the severity of depressive symptoms change at baseline and follow-up (threemonth and six-month) across two treatment conditions (i.e., integrated care and enhanced specialty referral), after adjusting for behavioral health covariates [depression type, 
current at-risk drinking, current anxiety, and suicidality], physical health covariates [chronic medical conditions], site location, and behavioral health treatment model.

Using the intercept-only model as the null model (i.e., statistical model without any predictors), the life stressors predictor variables were added to the second model to address research question 2 . The life stressors variables were entered to examine to what extent is the change in the severity of depressive symptoms at baseline and follow-up (three-month and six-month) across two treatment conditions (i.e., integrated care and enhanced specialty referral) related to frequency of life stressors at baseline, after adjusting for covariates.

To develop the third model, using the intercept-only as the null model, the life stressors predictor variables were removed and replaced with the gender variable to address research question 3 . This model examined to what extent is the change in the severity of depressive symptoms at baseline and follow-up (three-month and six-month) across two treatment conditions (i.e., integrated care and enhanced specialty referral) related to patients' gender, after adjusting for covariates.

To build the final model, using the intercept-only model as the null model, the life stressor variables, the gender variable, and the interaction terms of gender and frequency of life stressors were added to the model to address research question 4. This model examined to what extent is the change in the severity of depressive symptoms at baseline and follow-up (three-month and six-month) across two treatment conditions (i.e., integrated care and enhanced specialty referral) related to the interaction between gender and life stressors after adjusting for covariates. 
For each model, two analytic decisions needed to be considered regarding specific variables before running the analysis. First, if the variable should be uncentered, groupcentered, or grand-centered (Bryk \& Raudenbush, 1992). Centering is commonly performed on level-one variables while centering the dependent variable is unlikely since it would make interpretations difficult (Tabachnick \& Fidell, 2012). Tabachnick and Fidell (2012) suggest that centering can facilitate interpretation when the value of zero for each predictor is not meaningful. Therefore, based on Tabachnick and Fidell's recommendations, all variables were left uncentered to improve the reliability of the estimates.

Second, if the intercept or slope of the variable should be defined as a fixed effect or random effect (Bryk \& Raudenbush, 1992). To address the research questions and adjust the within-subject variation and correlation, a random-intercept model was chosen as the appropriate model. Therefore, to define the variables as fixed or random was dependent upon the assumptions of a random-intercept model. The intercepts in these models were allowed to vary; therefore, the depression severity scores for each individual were predicted by the intercept, which varied across the groups. The random-intercept model assumes that slopes are fixed; therefore, all remaining variables were defined as fixed variables (Bryk \& Raudenbush, 1992; Garson, 2013).

The Akaike Information Criterion (AIC) and Bayesian Information Criterion (BIC) of each statistical model will be reported in the following section. The AIC and BIC are measures used for model selection in non-nested data (Akaike, 1973). BIC is adjusted by the number of predictors in the model while the AIC does not adjust (Akaike, 
1973). Therefore, to ensure accuracy, both the AIC and BIC were reviewed. The AIC and the BIC with the lowest value suggests the best fit of the data to the population parameters (Akaike, 1973). Since all of the control variables were retained in each of statistical models despite lack of statistical significance for the purposes of answering the research questions appropriately, each of the AIC's and BIC's produced from each research question were compared to find which model demonstrated the best fit (see Table 4.6).

The following details the research aims and hypotheses and the findings from the statistical analyses.

Research Question 1: To what extent does the severity of depressive symptoms change at baseline and follow-up (three-month and six-month) across two treatment conditions (i.e., integrated care and enhanced specialty referral); after adjusting for behavioral health covariates [current at-risk drinking, current anxiety, and suicidality], physical health covariates [chronic medical conditions], site location and behavioral health treatment model? Hypothesis 1: After controlling for covariates, primary care patients diagnosed with major depressive disorder at baseline will show a decline in the severity of their depressive symptoms in both treatment conditions at follow-up.

\section{Extent severity of depressive symptoms change at baseline, and three-month}

and six-month follow-up. Table 4.7 shows individual differences in the intercepts (average depression scores for participants from baseline, three-month, to six-month) and the statistically significant residual while adjusting for behavioral health and physical health covariates. 
Major depressive disorder. The intercept-only model with the sample diagnosed with major depressive disorder at baseline successfully converged and produced Akaike's Information Criterion (AIC) of 5331.64 and Bayesian information criterion (BIC) of 5419.35. In this model, depression severity was related to six-month CES-D scores (Radloff) for individuals diagnosed with major depressive disorder. At six-month individuals diagnosed with major depressive disorder scored on average 1.57 points lower on the CES-D (Radloff) than at baseline, $t(2)=-2.36, p<.05$.

Depression severity as measured by the CES-D (Radloff) was also positively related to reports of suicidal ideation and death ideation as measured by the Paykel Suicide Questionnaire (Paykel et al., 1974) for individuals diagnosed with major depressive disorder. From baseline, three-month, to six-month participants who reported suicidal ideation scored on average 4.4 points higher on the CES-D (Radloff) than participants did not report suicidal ideation or death ideation from baseline, three-month, to six-month, $t(2)=3.69, p<.05$. From baseline, three-month, to six-month participants who reported death ideation scored on average 3.16 points higher on the CES-D (Radloff) than participants did not report suicidal ideation or death ideation from baseline, threemonth, to six-month, $t(2)=4.75, p<.05$.

Depression severity was also positively related to a diagnosis of current anxiety disorder NOS and panic disorder as measured by baseline scores on the MINI-Anxiety (Sheehan et al., 1998) for individuals diagnosed with major depressive disorder. From baseline, three-month, to six-month participants with a diagnosis of current anxiety disorder NOS scored on average 3.39 points higher on the CES-D (Radloff) than 
participants did not have any current anxiety diagnosis, $t(3)=4.01, p<.05$. From baseline, three-month, to six-month participants with a diagnosis of current panic disorder scored on average 4.99 points higher on the CES-D (Radloff) than participants did not have any current anxiety diagnosis, $t(3)=2.20, p<.05$.

The size and direction of the relationship suggest that participation at Sunset Park, NY, Chinatown, NY, and Philadelphia, PA was associated with higher severity of depression based on CES-D scores (Radloff) in comparison to San Francisco, California. The research site Sunset Park, New York was positively associated to depression severity, $t(4)=8.92, p<.05$, in which participants at this site scored on average 11.88 points higher on the CES-D (Radloff) than participants from the San Francisco, California site (reference group). The research site Chinatown, New York was positively associated to depression severity, $t(4)=6.25, p<.05$, in which participants at this site scored on average 6.84 points higher on the CES-D (Radloff) than participants from the San Francisco, California site (reference group). The research site Philadelphia, PA was also positively associated to depression severity, $t(4)=2.29, p<.05$, in which participants at this site scored on average 3.55 points higher on the CES-D (Radloff) than participants from the San Francisco, California site (reference group).

No statistically significant associations were found between depression severity and the following variables: (a) diagnosis of at-risk drinking, (b) physical health conditions (i.e., diabetes, high blood pressure, and heart trouble), (c) and behavioral health treatment model. 
The intraclass correlation (ICC) is the ratio between the cluster variance and total variance of the statistical model (Shrout \& Fleiss, 1979). The ICC describes how much the groups in the statistical model are similar to each other. The intercept-only model is used to calculate the ICC (Garson, 2013). In this intercept-only model, $\sigma^{2}=29.27$ and $\tau=$ 47.72, which resulted in an ICC of .3801 . This result suggests that $38 \%$ of the variance in CES-D scores is at the group level and $62 \%$ is at the individual level.

Subthreshold depression. The intercept-only model with the sample diagnosed with subthreshold depression at baseline successfully converged and produced Akaike's Information Criterion (AIC) of 2704.88 and Bayesian Information Criterion (BIC) of 2781.09. In this model, depression severity was not related to three-month and six-month CES-D scores (Radloff) for individuals diagnosed with major depressive disorder.

Depression severity as measured by the CES-D (Radloff) was also positively related to reports of suicidal ideation and death ideation as measured by the Paykel Suicide Questionnaire (Paykel et al., 1974) for individuals diagnosed with subthreshold depression. From baseline, three-month, to six-month participants who reported suicidal ideation scored on average 4.43 points higher on the CES-D (Radloff) than participants did not report suicidal ideation or death ideation from baseline, three-month, to sixmonth, $t(2)=3.23, p<.05$. From baseline, three-month, to six-month participants who reported death ideation scored on average 2.87 points higher on the CES-D (Radloff) than participants did not report suicidal ideation or death ideation from baseline, three-month, to six-month, $t(2)=3.67, p<.05$. 
Depression severity was also positively related to a diagnosis of current generalized anxiety disorder, current anxiety disorder NOS, and panic disorder as measured by baseline scores on the MINI-Anxiety (Sheehan et al., 1998) for individuals diagnosed with subthreshold depression. From baseline, three-month, to six-month participants with a diagnosis of current generalized anxiety Disorder scored on average 5.9 points higher on the CES-D (Radloff) than participants did not have any current anxiety diagnosis, $t(3)=3.79, p<.05$. Also, from baseline, three-month, to six-month participants with a diagnosis of current anxiety disorder NOS scored on average 5.22 points higher on the CES-D (Radloff) than participants did not have any current anxiety diagnosis, $t(3)=5.14, p<.05$. Also, from baseline, three-month, to six-month participants with a diagnosis of current panic disorder scored on average 12.19 points higher on the CES-D (Radloff) than participants did not have any current anxiety diagnosis, $t(3)=$ $3.70, p<.05$.

The size and direction of the relationship suggest that participation at Sunset Park, NY and Chinatown, NY was associated with higher severity of depression based on CESD scores (Radloff) in comparison to San Francisco, California. The research site Sunset Park, New York was positively associated to depression severity, $t(4)=2.94, p<.05$, in which participants at this site scored on average 4.68 points higher on the CES-D (Radloff) than participants from the San Francisco, California site (reference group). The research site Chinatown, New York was positively associated to depression severity, $t$ (4) $=2.17, p<.05$, in which participants at this site scored on average 2.26 points higher on 
the CES-D (Radloff) than participants from the San Francisco, California site (reference group).

No statistically significant associations were found between depression severity and the following variables: (a) diagnosis of at-risk drinking (b) physical health conditions (i.e., diabetes, high blood pressure, and heart trouble), (c) time, and (d) behavioral health treatment model.

In this intercept-only model, $\sigma^{2}=15.94$ and $\tau=26.59$, which resulted in an ICC of .3747. This result suggests that $37 \%$ of the variance in CES-D scores is at the group level and $63 \%$ is at the individual level.

No depression. The intercept-only model with the sample that did not have a diagnose of depression at baseline successfully converged and produced Akaike's Information Criterion (AIC) of 3826.35 and Bayesian Information Criterion (BIC) of 3909.11. In this model, depression severity was also positively related to six-month and three-month CES-D scores (Radloff) for individuals not diagnosed with depression. At six-month individuals not diagnosed with depression scored on average 4.38 points higher on the CES-D (Radloff) than at baseline, $t(2)=5.45, p<.05$. Further, at threemonth individuals diagnosed with major depressive disorder scored on average 4.83 points higher on the CES-D (Radloff) than at baseline, $t(2)=6.10, p<.05$.

Depression severity was also positively related to a diagnosis of current anxiety disorder NOS and generalized anxiety disorder as measured by baseline scores on the MINI-Anxiety (Sheehan et al., 1998) for individuals not diagnosed with depression. From 
baseline, three-month, to six-month participants with a diagnosis of current anxiety disorder NOS scored on average 3.97 points higher on the CES-D (Radloff) than participants did not have any current anxiety diagnosis, $t(3)=3.50, p<.05$. Also, from baseline, three-month, to six-month participants with a diagnosis of generalized anxiety disorder scored on average 5.48 points higher on the CES-D (Radloff) than participants did not have any current anxiety diagnosis, $t(3)=3.39, p<.05$.

Depression severity was negatively related to a diagnosis of at-risk drinking as measured by baseline, three-month, and six-month scores on the Alcohol Consumption Questionnaire (Sobell et al., 1988). From baseline, three-month, to six-month participants with a diagnosis of at-risk drinking scored on average 5.35 points lower on the CES-D (Radloff) than participants did not have a diagnosis of at-risk drinking, $t(1)=-6.53$, $p<.05$.

The size and direction of the relationship suggest that participation at Chinatown, New York was positively associated to depression severity, $t(4)=-3.28, p<.05$, in which participants at this site scored on average 3.32 points lower on the CES-D (Radloff) than participants from the San Francisco, CA site (reference group).

No statistically significant associations were found between depression severity and the following variables: (a) suicidal ideation or death ideation, (b) physical health conditions (i.e., diabetes, high blood pressure, and heart trouble), (c) suicidality and (d) behavioral health treatment models. 
In this intercept-only model, $\sigma^{2}=14.94$ and $\tau=24.80$, which resulted in an ICC of .3759. This result suggests that $38 \%$ of the variance in CES-D scores is at the group level and $62 \%$ is at the individual level.

Research Question 2: To what extent is the change in the severity of depressive symptoms at baseline and follow-up (three-month and six-month) across two treatment conditions (i.e., integrated care and enhanced specialty referral) related to frequency of life stressors at baseline after adjusting for covariates? Hypothesis 2a: Patients who experience life stressors will report greater severity of depressive symptoms at baseline among primary care patients diagnosed with major depressive disorder. Hypothesis $2 b$ : Patients reporting life stressors at baseline will experience less of a decline in the severity of their depressive symptoms in both treatment conditions at follow-up.

\section{Extent depression severity is related to frequency of life stressors at baseline,}

and three-month and six-month follow-up. Table 4.8 shows baseline, three-month, and six-month differences in the intercepts (severity of depression scores for participants) as related to the frequency of life stressors and the statistically significant residual while adjusting for behavioral health and physical health covariates.

Major depressive disorder. This model successfully converged and produced Akaike's Information Criterion (AIC) of 5348.2 and Bayesian Information Criterion (BIC) of 5519. This model suggests there was no influence of life stressors from baseline, three-month, to six-month. This model suggests that on average, participants who had a 
diagnosis of major depressive disorder who reported "loss of family and friends" as a life stressor had 2.56 lower scores on the CES-D (Radloff), $t(2)=-2.26, p<.05$.

Depression severity as measured by the CES-D (Radloff) was also positively related to reports of death ideation and suicidal ideation as measured by the Paykel Suicide Questionnaire (Paykel et al., 1974). From baseline, three-month, to six-month participants who reported death ideation scored on average 3.25 points higher on the CES-D (Radloff) than participants did not report any death ideation or suicidal ideation from baseline, three-month, to six-month, $t(2)=4.84, p<.05$. Also, from baseline, threemonth, to six-month participants who reported suicidal ideation scored on average 4.44 points higher on the CES-D (Radloff) than participants did not report any death ideation or suicidal ideation from baseline, three-month, to six-month, $t(2)=3.68, p<.05$.

Depression severity was also positively related to a diagnosis of current anxiety disorder NOS and panic disorder as measured by baseline scores on the MINI-Anxiety (Sheehan et al., 1998). From baseline, three-month, to six-month participants with a diagnosis of current anxiety disorder NOS scored on average 3.34 points higher on the CES-D (Radloff) than participants did not have any current anxiety diagnosis, $t(3)=$ $3.92, p<.05$. From baseline, three-month, to six-month participants with a diagnosis of panic disorder scored on average 5.65 points higher on the CES-D (Radloff) than participants did not have any current anxiety diagnosis, $t(3)=2.49, p<.05$.

The research sites Sunset Park, NY, Chinatown, NY, and Philadelphia, PA were positively associated to depression severity $(p<.05)$, in which participants at this site scored on average 11.53 (Sunset Park, NY), 6.42 (Chinatown, NY), and 4.18 
(Philadelphia, PA) points higher on the CES-D (Radloff) from baseline, three-month, and six-month compared to participants from the San Francisco, CA site, $t(4)=8.34, p<.05, t$ (4) $=5.43, p<.05$., and $t(4)=2.60, p<.05$, respectively.

No statistically significant associations were found between depression severity and the following variables: (a) specific life stressors (i.e., lack of activities/work, money issues, medical illness, loss of friends or family, and family issues, (b) physical health conditions (i.e., diabetes, high blood pressure, or heart trouble), (c) at-risk drinking, (d) life stressors and time, and (e) behavioral health treatment model.

Subthreshold depression. This model successfully converged and produced Akaike's Information Criterion (AIC) of 2727.24 and Bayesian Information Criterion (BIC) of 2875.66. This model suggests there was no influence of life stressors from baseline, three-month, to six-month.

Depression severity as measured by the CES-D (Radloff) was positively related to reports of death ideation and suicidal ideation as measured by the Paykel Suicide Questionnaire (Paykel et al., 1974). From baseline, three-month, to six-month participants who reported death ideation scored on average 2.81 points higher on the CES-D (Radloff) than participants did not report any death ideation or suicidal ideation from baseline, three-month, to six-month, $t(2)=3.59, p<.05$. Also, from baseline, threemonth, to six-month participants who reported suicidal ideation scored on average 4.48 points higher on the CES-D (Radloff) than participants did not report any death ideation or suicidal ideation from baseline, three-month, to six-month, $t(2)=3.29, p<.05$. 
Depression severity was also positively related to a diagnosis of current anxiety disorder NOS, generalized anxiety disorder, and panic disorder as measured by baseline scores on the MINI-Anxiety (Sheehan et al., 1998). From baseline, three-month, to sixmonth participants with a diagnosis of current anxiety disorder NOS scored on average 5.34 points higher on the CES-D (Radloff) than participants did not have any current anxiety diagnosis, $t(3)=5.21, p<.05$. Also, from baseline, three-month, to six-month participants with a diagnosis of current generalized anxiety disorder scored on average 5.60 points higher on the CES-D (Radloff) than participants did not have any current anxiety diagnosis, $t(3)=3.56, p<.05$. Further, from baseline, three-month, to six-month participants with a diagnosis of panic disorder scored on average 11.76 points higher on the CES-D (Radloff) than participants did not have any current anxiety diagnosis, $t(3)=$ $3.52, p<.05$.

The research sites Sunset Park, NY and Chinatown, NY were positively associated to depression severity $(p<.05)$, in which participants at this site scored on average 5.38 (Sunset Park, NY) and 2.28 (Chinatown, NY) points higher on the CES-D (Radloff) from baseline, three-month, and six-month compared to participants from the San Francisco, CA site, $t(4)=3.20, p<.05$ and $t(4)=2.11, p<.05$, respectively.

No statistically significant associations were found between depression severity and the following variables: (a) specific life stressors (i.e., family issues, money issues, medical illness, moving, and loss of family or friends, (b) physical health conditions (i.e., diabetes, high blood pressure, or heart trouble), (c) at-risk drinking, (d) life stressors and 
time, (e) some sites including Philadelphia, PA and Rochester, NY, and (f) behavioral health treatment model.

No depression. This model successfully converged and produced Akaike's Information Criterion (AIC) of 3817.13 and Bayesian Information Criterion (BIC) of 3978.31. This model suggests that life stressor "loss of family and friends" was related to depression severity at three-month and six-month. Participants who did not have a diagnosis of depression who reported "loss of family and friends" as a life stressor had 6.01 points higher scores on the CES-D (Radloff) at three-month, $t(2)=3.61, p<.05$ and 5.57 points higher at six-month, $t(2)=3.33, p<.05$, compared to baseline. This model also suggests that life stressor "medical illness" was related to depression severity at three-month and six-month. Participants who did not have a diagnosis of depression who reported "medical illness" as a life stressor had 6.03 points lower scores on the CES-D (Radloff) at three-month, $t(2)=-3.82, p<.05$ and 5.89 points lower six-month, $t(2)=-$ $3.78, p<.05$ compared to baseline scores.

Depression severity was also positively related to a diagnosis of current anxiety disorder NOS and current generalized anxiety disorder as measured by baseline scores on the MINI-Anxiety (Sheehan et al., 1998). From baseline, three-month, to six-month participants with a diagnosis of current anxiety disorder NOS scored on average 3.77 points higher on the CES-D (Radloff) than participants did not have any current anxiety diagnosis, $t(3)=3.29, p<.05$. Also, from baseline, three-month, to six-month participants with a diagnosis of current generalized anxiety scored on average 4.87 points higher on 
the CES-D (Radloff) than participants did not have any current anxiety diagnosis, $t(3)=$ $3.12, p<.05$.

Depression severity was negatively related to a diagnosis of at-risk drinking as measured by baseline, three-month, and six-month scores on the Alcohol Consumption Questionnaire (Sobell et al., 1988) among individuals who did not have a diagnosis of depression. From baseline, three-month, to six-month participants with a diagnosis of atrisk drinking scored on average 5.41 points lower on the CES-D (Radloff) than participants did not have a diagnosis of at-risk drinking, $t(1)=-6.66, p<.05$.

The research site Chinatown, New York was associated to depression severity $(p<.05)$, in which participants at this site scored on average 4.78 points lower on the CES-D (Radloff) from baseline, three-month, and six-month compared to participants from the San Francisco, CA site, $t(4)=-4.13, p<.05$.

No statistically significant associations were found between depression severity and the following variables: (a) specific life stressors (i.e., money issues, medical illness, moving, (b) physical health conditions (i.e., diabetes, high blood pressure, or heart trouble), (c) some sites including Sunset Park, New York, Rochester, New York, and Philadelphia, Pennsylvania, (d) suicidality, and (e) behavioral health treatment group.

Research Question 3: To what extent is the change in the severity of depressive symptoms at baseline and follow-up (three-month and six-month) across two treatment conditions (i.e., integrated care and enhanced specialty referral) related to patients' gender, after adjusting for covariates? Hypothesis 3: Males will report a greater decline 
in depressive symptoms than females at baseline and follow-up (three-month and sixmonth) across two treatment conditions (i.e., integrated care and enhanced specialty referral).

\section{Extent depression severity is related to gender at baseline, and three-month}

and six-month follow-up. Table 4.9 shows the impact of gender on baseline, threemonth, and six-month differences in scores on the CES-D (Radloff) among participants. Also reported is the statistically significant residual.

Major depressive disorder. This model successfully converged and produced Akaike's Information Criterion (AIC) of 5329.87 and Bayesian Information Criterion (BIC) of 5431.42. This model suggests that depression severity on average is not related to female gender, $t(1)=-.1 .14, p>.05$. However, females had 3.98 higher depression severity scores than men at six-month, $t(1)=2.77, p>.05$

Depression severity as measured by the CES-D (Radloff) was also positively related to reports of death ideation and suicidal ideation as measured by the Paykel Suicide Questionnaire (Paykel et al., 1974) among individuals diagnosed with major depressive disorder. From baseline, three-month, to six-month participants who reported death ideation scored on average 3.22 points higher on the CES-D (Radloff) than participants did not report any suicidal ideation or death ideation from baseline, threemonth, to six-month, $t(2)=4.86, p<.05$. Also, from baseline, three-month, to six-month participants who reported suicidal ideation scored on average 4.5 points higher on the CES-D (Radloff) than participants did not report any suicidal ideation or death ideation from baseline, three-month, to six-month, $t(2)=3.77 p<.05$. 
Depression severity was also positively related to a diagnosis of current anxiety disorder NOS and panic disorder as measured by baseline scores on the MINI-Anxiety (Sheehan et al., 1998) among individuals diagnosed with major depressive disorder. From baseline, three-month, to six-month participants with a diagnosis of current anxiety disorder NOS scored on average 3.66 points higher on the CES-D (Radloff) than participants did not have any current anxiety diagnosis, $t(3)=3.99, p<.05$. From baseline, three-month, to six-month participants with a diagnosis of panic disorder scored on average 4.96 points higher on the CES-D (Radloff) than participants did not have any current anxiety diagnosis, $t(3)=2.20, p<.05$.

The research sites Sunset Park, NY, Chinatown, NY, and Philadelphia, PA were positively associated to depression severity. Participants at the Sunset Park, New York site scored on average 11.97 points higher on the CES-D (Radloff) from baseline, threemonth, and six-month compared to participants from the San Francisco, CA site, $t$ (4) $=8.99, p<.05$. Participants at the Chinatown, New York site scored on average 6.78 points higher on the CES-D (Radloff) from baseline, three-month, and six-month compared to participants from the San Francisco, CA site, $t(4)=6.19 p<.05$. Further, participants at the Philadelphia, PA site scored on average 3.53 points higher on the CES$\mathrm{D}$ (Radloff) from baseline, three-month, and six-month compared to participants from the Philadelphia, Pennsylvania site, $t(4)=2.28 p<.05$.

No statistically significant associations were found between depression severity and the following variables: (a) average depression severity scores based on gender, (b) 
physical health conditions (i.e., diabetes, high blood pressure, heart trouble, and (c) behavioral health treatment models.

Subthreshold depression. This model successfully converged and produced Akaike's Information Criterion (AIC) of 2709.63 and Bayesian Information Criterion (BIC) of 2797.87. This model suggests that depression severity is not related to female gender, $t(1)=.273, p>.05$. For each time point, male and female depression severity scores were not statistically significant.

Depression severity as measured by the CES-D (Radloff) was also positively related to reports of suicidal ideation and death ideation as measured by the Paykel Suicide Questionnaire (Paykel et al., 1974) among individuals diagnosed with subthreshold depression. From baseline, three-month, to six-month participants who reported suicidal ideation scored on average 4.47 points higher on the CES-D (Radloff) than participants did not report any suicidal ideation or death ideation from baseline, three-month, to six-month, $t(2)=3.25, p<.05$. From baseline, three-month, to six-month participants who reported death ideation scored on average 2.78 points higher on the CES-D (Radloff) than participants did not report any suicidal ideation or death ideation from baseline, three-month, to six-month, $t(2)=3.55, p<.05$.

Depression severity was also positively related to a diagnosis of current anxiety disorder NOS, generalized anxiety disorder, and panic disorder as measured by baseline scores on the MINI-Anxiety (Sheehan et al., 1998) among individuals diagnosed with subthreshold depression. From baseline, three-month, to six-month participants with a 
diagnosis of current anxiety disorder scored NOS on average 5.19 points higher on the CES-D (Radloff) than participants did not have any current anxiety diagnosis, $t(3)=$ 5.13, $p<.05$. Also, from baseline, three-month, to six-month participants with a diagnosis of current generalized anxiety disorder scored on average 5.77 points higher on the CES$\mathrm{D}$ (Radloff) than participants did not have any current anxiety diagnosis, $t(3)=3.68$, $p<.05$. Also, from baseline, three-month, to six-month participants with a diagnosis of panic disorder scored on average 11.88 points higher on the CES-D (Radloff) than participants did not have any current anxiety diagnosis, $t(3)=3.59, p<.05$.

The research sites Sunset Park, NY and Chinatown, NY were positively associated to depression severity. Participants at the Sunset Park, New York site scored on average 4.73 points higher on the CES-D (Radloff) from baseline, three-month, and six-month compared to participants from the San Francisco, CA site, $t(4)=2.96, p<.05$. Participants at the Chinatown, New York site scored on average 2.18 points higher on the CES-D (Radloff) from baseline, three-month, and six-month compared to participants from the San Francisco, CA site, $t(4)=2.09 p<.05$.

No statistically significant associations were found between depression severity and the following variables: (a) average depression severity scores based on gender, (b) depression severity over time, (c) physical health conditions (i.e., diabetes, high blood pressure, heart trouble), (d) some sites, or (e) behavioral health treatment models.

No depression. This model successfully converged and produced Akaike's Information Criterion (AIC) of 3831.07 and Bayesian Information Criterion (BIC) of 3926.91. This model suggests that depression severity is not related to female gender, $t$ 
(1) $=-.266, p>.05$. For each time point, male and female depression severity scores were not statistically significant.

Depression severity was also positively related to a diagnosis of current anxiety disorder NOS and generalized anxiety disorder as measured by baseline scores on the MINI-Anxiety (Sheehan et al., 1998) among individuals not diagnosed depression. From baseline, three-month, to six-month participants with a diagnosis of current anxiety disorder NOS scored on average 4 points higher on the CES-D (Radloff) than participants who did not have any current anxiety diagnosis, $t(3)=3.34, p<.05$. Also, from baseline, three-month, to six-month participants with a diagnosis of current generalized anxiety disorder scored on average 5.61 points higher on the CES-D (Radloff) than participants who did not have any current anxiety diagnosis, $t(3)=3.46, p<.05$.

Depression severity was negatively related to a diagnosis of at-risk drinking as measured by baseline, three-month, and six-month scores on the Alcohol Consumption Questionnaire (Sobell et al., 1988) among individuals who did not have a diagnosis of depression. From baseline, three-month, to six-month participants with a diagnosis of atrisk drinking scored on average 5.24 points lower on the CES-D (Radloff) than participants did not have a diagnosis of at-risk drinking, $t(1)=-6.27, p<.05$.

Depression severity was also positively related to three-month and six-month CES-D scores (Radloff) for individuals not diagnosed with depression. At three-month individuals not diagnosed with depression scored on average 4.87 points higher on the CES-D (Radloff) than at baseline. At six-month individuals not diagnosed with depression scored on average 4.69 points higher on the CES-D (Radloff) than at baseline. 
Participants at the Chinatown, New York site scored on average 3.29 points lower on the CES-D (Radloff) from baseline, three-month, and six-month compared to participants from the San Francisco, CA site, $t(4)=-3.26, p<.05$.

No statistically significant associations were found between depression severity and the following variables: (a) average depression severity scores based on gender, (b) physical health conditions (i.e., diabetes, high blood pressure, heart trouble), (c) suicidality, or (d) behavioral health treatment condition.

Research Question 4: To what extent is the change in the severity of depressive symptoms at baseline and follow-up (three-month and six-month) across two treatment conditions (i.e., integrated care and enhanced specialty referral) related to the interaction between gender and life stressors after adjusting for covariates? Hypothesis 4a: Females with life stressors at baseline will show less of a decline in the severity of depressive symptoms at follow-up compared males diagnosed with major depressive disorder who report experiencing life stressors at baseline.

\section{Interaction between gender and frequency of life stressors by depression type} at baseline, and three-month and six-month follow-up. Table 4.10 examines the impact of the interaction between gender and frequency of life stressors on baseline, three-month, and six-month differences in scores on the CES-D (Radloff) among participants who had a baseline diagnosis of major depressive disorder or subthreshold depression. 
Major depressive disorder. This model successfully converged and produced Akaike's Information Criterion (AIC) of 5359.35 and Bayesian Information Criterion (BIC) of 5627.08. The following is a description of the findings.

Family issues. Females who had a baseline diagnosis of major depressive disorder who reported "family issues" as a life stressor at baseline reported higher depression severity at baseline, $t(2)=1.93, p=05$. At baseline, females who had a diagnosis of major depressive disorder reported an average of 4.51 higher scores on the CES-D (Radloff, 1977) compared to males who did reported "family issues" as a life stressor at baseline.

Medical illness. Females who had a baseline diagnosis of major depressive disorder who reported "medical illness" as a life stressor reported higher depression severity at three-month, $t(2)=2.04, p<.05$. At three-month, females who had a diagnosis of major depressive disorder reported an average of 5.75 higher scores on the CES-D (Radloff, 1977) compared to males who reported "medical illness" as a life stressor.

Lack of activities/work. Females who had a baseline diagnosis of major depressive disorder who reported "lack of activities/work" as a life stressor at baseline was related to depression severity at six-month, $t(2)=2.01, p<.05$. At six-month, females who had a baseline diagnosis of major depressive disorder who reported "lack of activities/work" at baseline had 6.24 lower scores on the CES-D (Radloff, 1977) compared to males who reported "lack of a activities" as a life stressor.

Money issues. Females who had a baseline diagnosis of major depressive disorder who reported "money issues" as a life stressor at baseline was related to depression 
severity at six-month, $t(2)=2.00, p<.05$. At six-month, females who had a diagnosis of major depressive disorder who reported "money issues" had an average of 6.15 higher scores on the CES-D (Radloff, 1977) compared to males who reported "money issues" as a life stressor.

Subthreshold depression. Table 4.10 examines the impact of the interaction between gender and frequency of life stressors on baseline, three-month, and six-month differences in scores on the CES-D (Radloff) among participants who had a diagnosis of subthreshold depression. Also reported is the statistically significant residual. This model produced Akaike's Information Criterion (AIC) of 2737.3 and Bayesian Information Criterion (BIC) of 5627.08. The following is a description of the findings.

Loss of family and friends. Males who had a baseline diagnosis of subthreshold depression who reported "loss of family and friends" as a life stressor reported lower depression severity at three-month, $t(2)=2.56, p<.05$. At three-month, males who had a baseline diagnosis of subthreshold depression reported an average of 9.34 lower scores on the CES-D (Radloff, 1977) compared to females who reported "loss of family and friends" as a life stressor.

Lack of activities/work. Females who had a baseline diagnosis of subthreshold depression who reported "lack of activities/work" as a life stressor at baseline was related to depression severity at three-month, $t(2)=-2.172, p<.05$. At three-month, females who had a baseline diagnosis of subthreshold depression who reported "lack of activities/work" at baseline had 8.26 lower scores on the CES-D (Radloff, 1977) compared to males who reported "lack of a activities" as a life stressor. 
No depression. This model produced Akaike's Information Criterion (AIC) of 3843.95 and Bayesian Information Criterion (BIC) of 4096.6. However, the participants who did not have a diagnosis of depression did not produce significantly significant results when examining the interaction between life stressors and gender.

\section{CHAPTER V. DISCUSSION}

This study was conducted to address the gap in knowledge on gender-specific differences in the correlates of depression and inform screening and early preventative interventions by: (1) examining gender-specific differences in the correlates of depression in older primary care patients based on baseline and longitudinal analyses; and (2) examining the longitudinal effects of biopsychosocial risk factors on depression treatment outcomes in two different models of behavioral health care, integrated care and enhanced referral. Current research has focused primarily on identifying and reducing the symptoms of depression for the general aging population, despite substantial limitations to characterizing depression using the symptom-based approach as described in chapter one. This study's results contribute significantly to current scientific knowledge by examining the foundational causes of depression in a person's development and the influence of contributory life span risk factors. Gender-specific differences in the correlates of depression were identified when the interactions between the individual and their environment were considered.

This study incorporated variables from both behavioral health and aging literature and integrated two existing perspectives: the life span perspective (Baltes, 1987; Baltes, 
Lindenberger, \& Staudinger, 2006) and the risk and resilience perspective (Rutter, 1987;

Saleebey, 1996) and examined how gender influenced the relationship between life

stressors and depression while accounting for the influence of psychological and

biological risk factors for depression in two different behavioral health treatment models.

Study results identified new directions for screening, assessment, and early preventative behavioral health interventions for both male and female older primary care patients.

This chapter provides a discussion of the results of the study. First, the findings of the study are described. Second, a discussion regarding the findings of the research questions is provided. Third, implications for social work and public health professionals, limitations of the current study, and recommendations for future studies are presented.

\section{Analysis of Dissertation Findings}

This study examined the influence of gender and life stressors on depression severity in two behavioral health treatment models at baseline, three-month, and sixmonth intervals among older primary care patients. Four research questions were examined. First, it was hypothesized that primary care patients diagnosed with major depressive disorder at baseline would show a decline in the severity of their depressive symptoms from baseline to three and six-month follow-up. There was support for this hypothesis when statistically significant results suggested that depression severity decreased at six-month for primary care patients diagnosed with major depressive disorder.

Second, it was hypothesized that (a) primary care patients who experience life stressors will report greater severity of depressive symptoms at baseline among primary 
care patients diagnosed with major depressive disorder, and (b) primary care patients reporting life stressors at baseline will experience less of a decline in the severity of their depressive symptoms. There was some support for this hypothesis as participants who did not have a diagnosis of depression yet reported life stressor "loss of family and friends" had higher depression severity compared to participants who did not have a diagnosis of depression and did not report life stressors.

Third, it was hypothesized that males would report a greater decline in depressive symptoms than females from baseline to three and six-month follow-up. There was some support for this hypothesis. A decline depression severity was not statistically significant for males, however, for females diagnosed with major depressive disorder, female gender was associated with higher depression severity at six-month compared to males.

Fourth, it was hypothesized that females with life stressors at baseline will show less of a decline in the severity of depressive symptoms at three and six month follow-up compared to males who report experiencing life stressors at baseline. There was support for this hypothesis when statistically significant results suggested that individuals who reported life stressors had higher depression severity at each time point; however, different life stressors affected males and females as related to depression severity.

The following sections describe the key findings for each research question.

\section{Changes in depression severity at baseline, three-month, and six-month.}

Descriptive statistics showed that depression severity decreased from baseline, to threemonth, and to six-month follow-up (see Table 4.1). When imputed into the HLM model 
and after adjusting for covariates, statistically significant results suggested that depression severity decreased at six-month for primary care patients diagnosed with major depressive disorder.

This finding is notable since previous research that examined depression outcomes in these two behavioral health treatment models using the PRISM-E database included all ten PRISM-E sites and included veterans in the sample (Krahn et al., 2006). Krahn et al. found a reduction in depression symptomology as measured by the CES-D (Radloff) in both behavioral health models among individuals diagnosed with major depressive disorder. The sample used in Krahn et al. (2006) included veterans unlike the present study that excluded veterans based on the unique differences in the VA health care system compared to the general population. Therefore, this study provides greater generalizability to the general population than the previous study conducted by Krahn et al.

Impact of life stressors on depression severity. According to Gitterman (2011) in social work practice, there are three defined categories of life stressors: (a) challenging life transitions and traumatic experiences; (b) environmental stresses; and (c) maladaptive interpersonal processes. For the purposes of this study, difficult life transitions and traumatic life events were examined since difficult life transitions and traumatic life events are both key risk factors for depression (Fiske et al., 2009). Life stressors were defined as "loss of family/friends", "lack of activities/work", "family issues", "money issues", "medical illness", and "moving/relocation". The study's findings on the impact 
of life stressors on depression severity suggested the greatest influence of life stressors on depression among participants who did not have a diagnosis of depression.

The study's findings suggest that participants who did not have a diagnosis of depression yet reported life stressors "loss of family and friends" had higher depression severity compared to participants who did not have a diagnosis of depression and did not report life stressors. This finding suggests that depression should be measured on a continuum. By viewing depression on a continuum, although participants did not have a diagnosis of depression they may have the greatest fluctuation in depression severity symptoms in response to a life stressor.

This statistical model had the best fit based on the AIC and BIC (see Table 4.6). However, to begin to examine gender-based differences in longitudinal depression treatment outcomes it is necessary to examine the interaction between life stressors and gender, despite the other model have a worse fit.

Impact of gender on depression severity. Findings from this study suggest that on average gender did not influence depression severity. However, for participants diagnosed with major depressive disorder female gender was associated with higher depression severity at six-month compared to males. This finding is consistent with other findings that suggest that females have longer bouts of depression than males (Kessler et al., 2003). Unlike prior studies that examined this relationship, this study is distinguished since gender was explored within primary care settings that used two separate behavioral health models from a large nationally representative sample of older adults. 
Interaction between gender and life stressors. This analysis examined the influence of gender and life stressors by depression type (i.e., no depression, major depressive disorder, and subthreshold depression). The results suggested that specific life stressors affected males and females differently, and females who experience specific life stressors "loss of family and friends", "family issues", "money issues", "medical illness" have higher depression severity when compared to males. Notable gender differences were found on life stressors and the implications are presented in the following sections.

Medical illness. For females, "medical illness" was related to higher depression severity for females who had a diagnosis of major depressive disorder. Chronic physical health conditions are correlated to depression for both males and females (Fiske et al., 2009); however, throughout the entirety of the life span, females have more physical health conditions than males (Anderson et al., 2001; Egede et al., 2002; Verbrugge, 1995). It is possible that older females are experiencing the cumulative effect of having multiple chronic medical conditions, and this cumulative risk has resulted in higher depression severity.

To further complicate the complexity of depression, according to the DSM-V, a major depressive disorder diagnosis cannot be established if depressive symptoms are due to a physical illness (American Psychiatric Association, 2013). Therefore, depression may be underdiagnosed if depression co-occurs with a physical illness and this may affect females more so than males based on this study's findings. 
Family issues. "Family issues" resulted in higher depression severity at baseline for females who had a diagnosis of major depressive disorder. An interaction with family members, friends, or groups and the community foster feelings of belongingness and acceptance and establishes or maintains healthy interpersonal relationships (Hagerty \& Patusky, 2003). However, males and females may experience social interactions with family differently, and therefore, place different value on experiencing negative "family issues". Research has suggested that females' interaction with family and other groups (i.e., friends, community, groups) affects their well-being more so than males (Warr, Butcher, \& Robertson, 2004). Further, in support of previous research, research has also suggested that female's survival rates increased with increases in social interactions while males survival rates was increased by solitary activities (Agahi \& Parker, 2008). This study's findings suggest that "family issues" may be an important component for early preventative interventions for females in primary care.

Lack of activities/work. "Lack of activities/work" resulted in lower depression severity for females who had a diagnosis of major depressive disorder or subthreshold depression. Engaging in activities throughout the life span is considered an essential part of successful aging (Adams, Leibbrandt, \& Moon, 2011). Many older adults who participate in social and leisure activities have increased feelings of well-being and decreased depression symptomology (Glass et al., 2006; Hong, Hasche \& Bowland, 2009; Janke \& Davey, 2006; Janke, Davey, \& Kleiber, 2006; Lampinen et al., 2006).

Perhaps, there are gender-based differences in the satisfaction one receives from engaging in activities and the relationship to depression severity. Males may place more 
value on activities throughout the life span. Engaging in activities may assist in role continuity and contribute to feelings of purpose and preservation of self-identity (Atchley, 1989; Lemon, Bengtson \& Peterson, 1972) more so for older males than females. For example, research has suggested that for males being retired increases the importance of participating in activities for well-being (Harlow \& Cantor, 1996).

Gender roles may explain the gender-based differences in "lack of activities/work" and depression severity. Gender roles are considered behaviors of males and females that vary by gender (Schaie \& Willis, 2011). Socialization and stereotypes influence gender roles through society's encouragement of stereotypical behavior and discouragement of behavior contradictory to these stereotypes (Schaie \& Willis, 2011).

Using the life span risk and resilience perspective, development occurs throughout the life span (Blazer \& Steffens, 2009; Santrock, 2011). Therefore, in older adulthood individuals are still developing, and their development is a product of dynamic interactions between the developing self and the environment throughout the entirety of the life span (Blazer \& Steffens, 2009; Santrock, 2011). Therefore, older adults focus on maintaining self-identity, meaning, and community (Schaie \& Willis, 2011), and this process includes maintaining gender-based roles established throughout their life span, which may include stereotypical behavior.

Research suggests that volunteering (Hong, Hasche \& Bowland, 2009) and engaging in social activities (i.e., religious activities such as attending church, exercising, talking on the telephone, going to restaurants, or watching sporting events) is associated with lower depression at baseline (Glass et al., 2006; Hong, Hasche, \& Bowland, 2009). 
Further, informal and formal leisure activities may also reduce depression (Janke \& Davey, 2006; Janke et al., 2006) such as community memberships or associations, religious activities, writing, or reading books or the newspaper (Lampinen et al., 2006). This study's findings suggest that encouraging engagement in meaningful activities throughout the life span may assist in maintaining or reducing depression severity, possibly more for males than females.

Money issues. "Money issues" was related to higher depression severity at sixmonth for females who had a diagnosis of major depressive disorder. Financial strain is a primary risk factor for depressive symptoms (Mojtabai \& Olfson, 2004; Umberson et al., 1992) and is the most frequently reported life stressor experienced by older adults (Fiske, Gatz \& Pedersen, 2003). This study's findings suggest that financial strain was related to depression severity among females more so than for male primary care patients.

"Money issues" and depression among older female primary care patients may be related to the kindling effects of experiencing other life stressors. For example, money issues may have been the result of the loss of a spouse. This study's findings suggest that "money issues" for may be an important component for early preventative interventions for females in primary care.

Loss of family or friends. "Loss of family or friends" was related to higher depression severity for women who had a diagnosis of subthreshold depression. Throughout the life span, the likelihood of losing a close family member, friend, or spouse increases as individuals mature into older adulthood. Studies have consistently found a link between depressive symptoms and bereavement in older adults (Cole \& 
Dendukuri, 2003; De Beurs et al., 2001; Prigerson et al., 1995). Compared to males, females have a greater likelihood of developing depression or depressive symptoms after the death of a spouse and will experience depressive symptoms for a longer duration than males (Fiske et al., 2009), which is consistent with the findings from this study. The disparity in depression severity between males and females suggests that this life stressor needs to be addressed in an intervention specifically for females.

\section{Additional Findings}

Additional findings were found after adjusting for covariates. Specifically, anxiety disorders (i.e., generalized anxiety disorder, panic disorder, and anxiety disorder NOS) and suicidal and death ideation were correlated with higher depression severity at each time point. These finding are consistent with the research on older adults that suggest anxiety (Thomas \& O’Brien, 2006; Blazer, 2009; Cuijpers et al., 2007), alcohol use disorders (Schonfeld et al., 2010; Glass et al., 1995; Dupree, Broskowski, \& Schonfeld, 1984), and suicidality (CDC, 2005) are common co-occurring conditions when an older adult experiences depression.

These findings suggest that early or preventative interventions should also focus on co-morbid conditions. For example, the relationship between co-morbid anxiety disorders and suicidality with depression suggests the need for older primary care patients to receive a battery of behavioral health assessments at intake to examine depression and 
other co-morbid conditions. Behavioral health assessments can be completed by an older primary care patient or be administered by medical social workers or nurses depending on the level of functionality of the patient. The assessments must have good reliability and validity with older adults; otherwise, screening may lead to inaccurate results.

\section{Gender-Based Risk and Resilience}

While examining the longitudinal effect of biopsychosocial risk factors on depression treatment outcomes in different models of behavioral health care(i.e., integrated care and enhanced referral), gender-specific differences in the correlates of depression emerged. This study found that females who reported "loss of family and friends", "family issues", "money issues", "medical illness" was related to higher depression severity compared to males whereas "lack of activities" was related to lower depression severity among females compared to males. These findings suggest that gender moderated the relationship between specific life stressors and depression severity similar to how a protective factor can impact a person's response to a problem and reduce the negative impact of a risk factor on a problem outcome (O'Connell, Boat, Warner, 2009; Rutter, 1979; Rutter, 1985). Gender may serve to establish and maintain positive mental health, promote recovery and/or diminish the negative effects of life stressors. Based on these findings, there is a need for gender-based depression screening and early preventative interventions. 


\section{Implications for Social Work and Public Health}

Given the increasing incidence and prevalence of depression among older adults, regardless of gender, this topic is relevant to researchers and providers in both the social work and public health fields. Primary care patients with depression are a vulnerable population that social workers and other mental health professionals need to focus on because primary care is the first point of contact for older adults seeking mental health treatment (Levkoff et al., 2004).

Research. Researchers also need to develop gender-specific interventions, and screening and assessment tools. Research has focused primarily on reducing the symptoms of depression for the general aging population, not the root causes of depression. The medical model characterizes depression using the symptoms-based approach while ignoring an integrated approach that examines the foundational causes of depression in an individual's development and the risk factors they have been exposed to in their life span. This study supports moving away from the symptoms-based approach to depression, and exploring the development gender-specific interventions that take into account the whole person and the underlying causes of depression, not solely treat symptoms.

Gender-based screening. Common depression screening tools used in primary care older adults include the Geriatric Depression Scale (Yesavage et al., 1982) and the MINI-Depression (Sheehan et al., 1998); however, these screening and assessment tools do not screen for life stressors; rather these tools focus on the traditional symptoms-based approach to screening for depression and not the whole person. Gender-based screening 
in primary care settings may alter the developmental pathways of depression in older adults. A new screening tool can be developed and psychometrically tested among older primary care patients that screens for life stressors. Future research can use life stressors from this study that were significant to guide future research that develops genderspecific screening tools.

Gender-based early preventative interventions. This study found life stressors may be a reliable predictor of depression for both women and men in either behavioral health treatment model. This study found that life stressors influence males basic comfort, stability, and survival whereas for life stressors influence females development, personal growth, and happiness; therefore, life stressors may be a useful component to include in gender-based screening and assessment tools for depression. Interventions within primary care settings that target these domains may influence depression severity among individuals who present with major depressive disorder, subthreshold depression, or no depression. Since individuals with no depression also have depression severity, preventative interventions should be explored.

The need for research to develop gender-based early preventative interventions is a significant issue for various health care fields. With the increasing number of adults reaching age 65 years and older, especially females (Vincent \& Velkoff, 2010), professionals in various health care settings including primary care, mental health, substance abuse, corrections and rehabilitation, assisted living facilities, social work, and nursing homes are likely to work with older adults who have depression. These gender- 
based early preventative interventions that focus on addressing life stressors may have significant effects on the development of depression in older adults.

Practice and teaching. While there is a lack of trained geriatric mental health professionals (Eden et al., 2012; Lennon, 2004), it is not known if the current workforce is trained on gender-based differences in depression. The lack of professionals who specialize in the treatment of depression in older adults has not only deterred the preparation of the mental health care system for the growing aging population but may also be related to misdiagnosis and underdiagnosis of depression in older adults. Addressing this issue requires a multi-systemic effort from mental health practitioners, researchers, and policy makers (Whiteman, Ruggiano, \& Thomlison, n.d.).

First, mental health providers must be knowledgeable about the different depression symptoms and males and females experience of life stress as a predictor of depression. Providers need to know about existing gender-specific interventions that can be incorporated into treatment planning. As the population of older adults grows, there is going to be a great need for providers to effectively diagnose and treat depression in older females and males. Social workers are in an ideal position to act as key interventionists and allow for the ease of translation of gender-based screening and interventions for older adults that focus on life stressors into the health care field, assisted living facilities, homebound care, or primary care offices.

With the aging population and the projected depression treatment needs of older adults, mental health providers need to screen both males and females using age and gender-appropriate tools. Although these instruments do not exist, education on how life stressors influence depression is a key early intervention component that needs to be 
taught to health care professionals (i.e., social workers, nurses, public health professionals, and primary care providers). Understanding life stressors as predictors of depression may assist providers in preventing or diagnosing depression in older males and females.

Current and future social workers need to have formal training on the genderspecific presentation of depression and also current gender-based depression treatments. National organizations including the Substance Abuse and Mental Health Services Administration and Agency for Healthcare Research and Quality need to emphasize the importance of depression in older adults and the large-scale impact these males and females will have on the health care system. These national organizations need to bring awareness to this issue and fund (a) the development of workbooks and curricula to be used when educating providers and (b) research on gender-based differences in depression in older adulthood that can be used to guide the design and development of gender and age-specific interventions, and screening and assessments.

National university accreditation agencies also need to emphasize the importance of this issue and bring awareness to university faculty and students. Through the support of national accreditation agencies, curricula in accredited medical, nursing, psychology, and social work programs can be required to focus on gender and age-specific clinical education in bachelors, masters, and doctorate programs. Clinical curricula should focus on the unique experience of depression in older males and females and the impact of life stress on depression, existing interventions, and adaptations of existing screening and assessment tools. 
Policy. Since human behavior is the result of interactions between an individual and their environment (Wormer, 2007) there is a need for our policies to support looking at the individual more holistically where the interactions between the individual and their environment are considered (Whiteman, Ruggiano, \& Thomlison, n.d.). This means promoting health through macro social work mechanisms in which policy considers the contextual factors associated with the development of depression.

Medicare can play an important role in moving the healthcare system towards care that focuses on the whole person, in which gender-based differences in the causes of depression are considered. Medicare is the largest single United State's purchaser of healthcare services (Centers for Medicaid and Medicare, 2005), and therefore, changes to Medicare can result in nationwide changes to the healthcare system.

To move the healthcare system away from the medical model and the limited symptoms-based approach, the following three objectives must be adopted by the Centers for Medicare and Medicaid Services: (1) develop targeted goals that focus on increasing the quality of mental health care through promoting the examination of the contextual factors related to depression for older adults; (2) encourage primary care physicians involvement in developing older adult behavioral health accreditation standards for primary care settings. The accreditation standards should maintain a focus on an integrated approach to care that considers the foundational causes of depression and the risk factors older adults have been exposed to in their life span; and (3) adopt reimbursement policy that encourages primary care physicians to utilize gender-bases screening and assessment and interventions. The reimbursement should offer primary 
care physicians additional financial reimbursement for care provided as determined by the Centers for Medicare and Medicaid Services.

\section{Integrated Life Span Risk and Resilience Perspective}

The integrated life span perspective (Baltes, 1987; Baltes, Lindenberger, \& Staudinger, 2006) and the risk and resilience perspective (Rutter, 1987; Saleebey, 1996) was useful in examining how gender influenced the relationship between life stressors and depression while accounting for the influence of psychological and biological risk factors for depression in two different behavioral health treatment models. The integrated life span risk and resilience perspective allowed for a more holistic examination of the whole person compared to traditional approaches that rely on the symptom-based approach to identifying and treating depression. The integrated life span risk and resilience perspective should be maintained in future social work studies when examining older adults as it allows for a comprehensive examination of the person and their interaction with their environment.

\section{Future Studies}

This study found a relationship among the influence between gender and life stressors on longitudinal depression treatment outcomes. However, future research on gender and life stressors is necessary. This same study can be conducted with the veterans in the sample using the original sample. The risk factors for depression in older veterans would need to be examined prior to running the analysis since these risk factors of this unique population may differ than the general population. This future study may 
provide more information regarding life stressors and gender and lead to new preventative and early interventions in the VA medical centers for both males and females.

A qualitative study should also be conducted to examine the influence of gender and life stressors on depression treatment outcomes. This study can be used to explore (a) what life stressors patients think are important in examining depression, (b) older adults perception of the influence of gender and life stressors on depression, and (c) examine older adults' perception of the need for gender-based interventions and satisfaction with existing mental health services.

\section{Limitations}

This study is characterized by several important strengths, particularly its large sample of adults aged 65 years and older in the United States representing different states and primary care settings. Further, in contrast to the majority of studies that have focused on major or minor depressive disorder (Katon et al., 1995; Weisner et al., 2001), this study focused on the continuum of mental health disorders that present among older adults in primary care settings, including major depressive disorder, dysthymia, anxiety, at-risk drinking, and comorbid mental health and substance abuse disorders.

There were also some limitations to using this data set that should be considered when interpreting its results. The study used secondary data from the Primary Care Research in Substance Abuse and Mental Health for Elderly (PRISM-E) study on older primary care patients. Since this was a secondary data analysis, the study could only include specific variables in the research question and hypotheses that were present in the 
already existing database. Therefore, variables such as specific treatment could not be examined, and the study is limited to the variables in the database.

How the participants interpreted the definitions of life stressors, "loss of family/friends", "lack of activities/work", "family issues", "money issues", "medical illness", and "moving/relocation", is not clear. For example, the study does not explain how the participants interpreted "moving/relocation", which could possibly be moving to a nursing home or a retirement home. Since this secondary data analysis included only quantitative data, it lacked the complexity of a mixed methods study in which perceptions of participants could be explored in-depth. Collecting qualitative data from participants in primary care settings at the original research sites may provide instrumental data about the perspectives of the participants.

Further the available variables in the original database were limited due to small sample size. Specifically, the physical health covariates selected for the study are associated with depression among females (i.e., diabetes or sugar sickness); (2) high blood pressure or hypertension; (3) heart trouble). Specific variables were associated with depression among both males and females including Parkinson's disease (Reijnders et al., 2008) and stroke (Park et al., 2007; Robinson \& Price, 1982); however, low sample sizes limited the inclusion of these variables.

This data set included adults aged 65 years and older in 1998 to 2004. Some researchers have suggested that baby boomers, persons born between 1946-1964, represent a different cohort of older adults than the previous generation (Han, Gfroerer, Colliver, \& Penne, 2009). Therefore, the older adults in this study may be affected by 
cohort effect and findings from this study can only be generalized to adults currently aged 74 years and older. While this created a limitation, it was also a great strength since individuals in the United States are living longer (Hoyert \& Xu, 2012) and these individuals aged 74 and older are still in need of targeted prevention and early intervention.

This study operationalized depression type (i.e., no depression, subthreshold depression, and major depressive disorder) and anxiety type (i.e., no anxiety, panic disorder, anxiety disorder NOS, generalized anxiety disorder) using DSM-IV (American Psychiatric Association, 2000); however, the DSM-IV has been updated to a new version, DSM-V (American Psychiatric Association, 2013) that mainly affected the diagnostic criteria for major depressive disorder.

Three noteworthy changes have happened in the DSM-V regarding major depressive disorder. First, major depressive disorder diagnoses cannot be established if depressive symptoms are due to a physical illness (American Psychiatric Association, 2013). Second, dysthymia is not a diagnosis in the DSM-V; rather, dysthymia has been supplanted by persistent depressive disorder. Persistent depressive disorder is a new diagnosis characterized by a combining chronic major depressive disorder and dysthymic disorder. This change was based on notion that there does not appear to be any scientifically meaningful differences between the two conditions (Rhebergen \& Graham, 2014). The following is the diagnostic criteria for individual diagnosed with persistent depressive disorder: 
(a) Depressed mood for most of the day, for more days than not, as indicated either by subjective account or observation by others, for at least 2 years. Note: In children and adolescents, mood can be irritable and duration must be at least 1 year; and presence, while depressed, of two (or more) of the following: (1) poor appetite or overeating; (2) insomnia or hypersomnia; (3) low energy or fatigue; (4) low self-esteem; (5) poor concentration or difficulty making decisions; (6) feelings of hopelessness.

(b) During the 2-year period (1 year for children or adolescents) of the disturbance, the person has never been without the above symptoms for more than 2 months at a time. (1) criteria for a major depressive disorder may be continuously present for 2 years; (2) there has never been a manic episode or a hypomanic episode, and criteria have never been met for cyclothymic disorder; (3)the disturbance is not better explained by a persistent schizoaffective disorder, schizophrenia, delusional disorder, or other specified or unspecified schizophrenia spectrum and other psychotic disorder; (4) the symptoms are not attributable to the physiological effects of a substance (e.g., a drug of abuse, a medication) or another medical condition (e.g., hypothyroidism); (5)the symptoms cause clinically significant distress or impairment in social, occupational, or other important areas of functioning. (American Psychiatric Association, 2013)

Third, "bereavement exclusion" has been removed from the major depressive disorder diagnosis. This exclusion was in effect if a person had major depressive symptoms within the first two months after the death of a loved one. This change to the DSM encourages mental health professionals to use their clinical judgment as to whether a patient with symptoms of major depressive disorder and who is also experiencing grief should be diagnosed with depression.

There were substantially less changes to anxiety disorders. There was one noteworthy change that affects the generalizability of this study, panic disorder is no longer associated with agoraphobia. The DSM-V now recognizes these as two separate 
disorders. The change was made since a significant number of people with agoraphobia do not have panic disorder symptoms.

All of these changes will impact the number of individuals who meet diagnostic criteria for major depressive disorder, persistent depressive disorder (dysthmia), and anxiety disorder (panic disorder). Since these changes to the DSM recently occurred the current screening tools will soon be outdated as social workers begin to use the DSM-V. Therefore, the timing to develop new screening and early preventative interventions based on the findings from this study is ideal.

\section{Summary}

In summary, this study found gender-based correlates of depression outcomes among older primary care patients. This study found that females who reported "loss of family and friends", "family issues", "money issues", and "medical illness" was related to higher depression severity compared to males whereas "lack of activities" was related to lower depression severity among females compared to males. These findings suggest that gender moderated the relationship between specific life stressors and depression severity among older primary care patients. Understanding the influence of gender and life stressors is key to reducing depression severity in older primary care patients. These findings inform further research on gender-based screening and gender-based early preventative depression interventions.

This study also looked at behavioral health risk factors, and after adjusting for behavioral health covariates and physical health covariates, anxiety disorders (i.e., generalized anxiety disorder, panic disorder, and anxiety disorder NOS), and suicidal and 
death ideation were correlated with higher depression severity at each time point. This finding is consistent with the research on older adults that suggests anxiety (Thomas \& O’Brien, 2006; Blazer, 2009; Cuijpers et al., 2007), alcohol use disorders (Schonfeld et al., 2010; Glass et al., 1995; Dupree, Broskowski, \& Schonfeld, 1984), and suicidality (CDC, 2005) are common occurrences when an older adult experiences depression.

This study was also distinguished from other studies in social work because (a) it included individuals who had a spectrum of mental health and substance use disorders, (b) PRISM-E is the largest randomized sample of alcohol use and depression in older primary care patients, (c) it focused on an underrepresented research population - older females, and (d) it used a nationally representative sample of older primary care patients. It was also distinguished by its exploration of gender differences in factors, which are associated with different experiences with depression.

\section{Conclusion}

Life stressors may be a reliable predictor of depression in older females and a useful component to include in screening and assessment tools. Since females respond differently to stress, a gender-based screening and assessment tool that does not focus solely on symptomatology may also be useful for older females. A life stress screening or assessment tool similar to the Social Readjustment Rating Scale (Holmes-Rahe, 1967) that evaluates life stress and its relationship to illnesses may be useful to predict depression in older females. However, more research needs to be conducted on the inclusion of life stress in gender-specific screening tools. 
The life span perspective can assist in identifying important gender-specific and age-appropriate components in the continuum of care that may alter developmental pathways for depression or reduce risk factors for worsening depression symptoms in both older males and females. Further, the absence of this perspective in the existing continuum of care may have negative clinical implications. 
Table 3.1

Characteristics of Primary Care Settings Involved in the Primary Care Research in Substance Abuse and Mental Health for the Elderly Study

\begin{tabular}{|c|c|c|c|c|c|c|c|c|c|c|}
\hline & \multicolumn{10}{|l|}{$\underline{\text { Site }}$} \\
\hline & 1 & 2 & 3 & 4 & 5 & 6 & 7 & 8 & 9 & 10 \\
\hline $\begin{array}{l}\text { Clinic } \\
\text { setting }\end{array}$ & Urban & $\begin{array}{l}\text { Urba } \\
\mathrm{n}\end{array}$ & $\begin{array}{l}\text { Urba } \\
\mathrm{n}\end{array}$ & $\begin{array}{l}\text { Suburba } \\
\text { n, urban }\end{array}$ & $\begin{array}{l}\text { Suburban, } \\
\text { urban }\end{array}$ & Rural & Urban & Urban & Urban & $\begin{array}{l}\text { Suburba } \\
n\end{array}$ \\
\hline Location & $\begin{array}{l}\text { San } \\
\text { Francisc } \\
\mathrm{o}, \mathrm{CA}\end{array}$ & $\begin{array}{l}\text { New } \\
\text { York }\end{array}$ & $\begin{array}{l}\text { New } \\
\text { York }\end{array}$ & $\begin{array}{l}\text { Rocheste } \\
\text { r, NY }\end{array}$ & $\begin{array}{l}\text { Philadelphi } \\
\text { a, PA }\end{array}$ & $\begin{array}{l}\text { White } \\
\text { River } \\
\text { Junction, } \\
\text { VT }\end{array}$ & $\begin{array}{l}\text { Miami, } \\
\text { FL }\end{array}$ & $\begin{array}{l}\text { Chicago, } \\
\text { IL }\end{array}$ & $\begin{array}{l}\text { Philadelphi } \\
\text { a, PA }\end{array}$ & $\begin{array}{l}\text { Madison } \\
\text {, WI }\end{array}$ \\
\hline Site type & $\mathrm{CHC}$ & $\mathrm{CHC}$ & $\mathrm{CHC}$ & $\begin{array}{l}\text { Hospital } \\
\text { network }\end{array}$ & $\begin{array}{l}\text { Hospital } \\
\text { network }\end{array}$ & $\begin{array}{l}\text { Veterans } \\
\text { Affairs } \\
\text { Medical } \\
\text { Center }\end{array}$ & $\begin{array}{l}\text { Veterans } \\
\text { Affairs } \\
\text { Medical } \\
\text { Center }\end{array}$ & $\begin{array}{l}\text { Veterans } \\
\text { Affairs } \\
\text { Medical } \\
\text { Center }\end{array}$ & $\begin{array}{l}\text { Veterans } \\
\text { Affairs } \\
\text { Medical } \\
\text { Center }\end{array}$ & $\begin{array}{l}\text { Veterans } \\
\text { Affairs } \\
\text { Medical } \\
\text { Center }\end{array}$ \\
\hline $\begin{array}{l}\text { Number } \\
\text { of } \\
\text { primary } \\
\text { care } \\
\text { clinics }\end{array}$ & 1 & 4 & 4 & 5 & 6 & 2 & 4 & 4 & 2 & 2 \\
\hline
\end{tabular}


Table 3.2

Characteristics of Non-Veterans Administration Primary Care Settings Involved in the Primary Care Research in Substance Abuse and Mental Health for the Elderly study

\begin{tabular}{|c|c|c|c|c|c|}
\hline Characteristics & 1 & 2 & 3 & 4 & 5 \\
\hline Clinic setting & Urban & Urban & Urban & Suburban/urban & Suburban/urban \\
\hline Location & $\begin{array}{l}\text { San } \\
\text { Francisco, } \\
\text { CA }\end{array}$ & $\begin{array}{l}\text { New York } \\
\text { (Chinatown) }\end{array}$ & $\begin{array}{l}\text { New York } \\
\text { (Sunset } \\
\text { Park) }\end{array}$ & Rochester, NY & Philadelphia, PA \\
\hline Site type & $\begin{array}{l}\text { Community } \\
\text { Health } \\
\text { Center }\end{array}$ & $\begin{array}{l}\text { Community } \\
\text { Health } \\
\text { Center }\end{array}$ & $\begin{array}{l}\text { Community } \\
\text { Health } \\
\text { Center }\end{array}$ & $\begin{array}{l}\text { Hospital } \\
\text { network }\end{array}$ & Hospital network \\
\hline $\begin{array}{l}\text { Number of } \\
\text { Primary Care } \\
\text { Clinics }\end{array}$ & 1 & 4 & 4 & 5 & 6 \\
\hline Sample size & 153 & 175 & 75 & 84 & 98 \\
\hline
\end{tabular}


Table 3.3

Characteristics of Integrated Care and Enhanced Specialty Referral Treatment for Major Depressive Disorder and Dysthymia

\begin{tabular}{|c|c|c|c|c|c|c|c|c|c|c|}
\hline \multirow[b]{2}{*}{ Characteristics } & \multicolumn{10}{|l|}{$\underline{\text { Site }}$} \\
\hline & 1 & 2 & 3 & 4 & 5 & 6 & 7 & 8 & 9 & 10 \\
\hline \multicolumn{11}{|c|}{ Typical treatment Integrated Care } \\
\hline \multicolumn{11}{|l|}{ MDD } \\
\hline primary & $\begin{array}{l}\text { Individu } \\
\text { al } \\
\text { therapy }\end{array}$ & $\begin{array}{l}\text { Individua } \\
1 \text { therapy }\end{array}$ & $\begin{array}{l}\text { Pharma } \\
\text { cology }\end{array}$ & $\begin{array}{l}\text { Pharmac } \\
\text { ology }\end{array}$ & $\begin{array}{l}\text { Pharma } \\
\text { cology }\end{array}$ & $\begin{array}{l}\text { Individu } \\
\text { al } \\
\text { therapy }\end{array}$ & $\begin{array}{l}\text { Pharma } \\
\text { cology }\end{array}$ & $\begin{array}{l}\text { Pharmaco } \\
\text { logy }\end{array}$ & $\begin{array}{l}\text { Pharmac } \\
\text { ology }\end{array}$ & $\begin{array}{l}\text { Pharma } \\
\text { cology }\end{array}$ \\
\hline secondary & Other & $\begin{array}{l}\text { Pharmaco } \\
\text { logy }\end{array}$ & $\begin{array}{l}\text { Individ } \\
\text { ual } \\
\text { therapy }\end{array}$ & $\begin{array}{l}\text { Individu } \\
\text { al } \\
\text { therapy }\end{array}$ & $\begin{array}{l}\text { Individ } \\
\text { ual } \\
\text { therapy }\end{array}$ & $\begin{array}{l}\text { Pharmac } \\
\text { ology }\end{array}$ & $\begin{array}{l}\text { Individ } \\
\text { ual } \\
\text { therapy }\end{array}$ & $\begin{array}{l}\text { Individua } \\
1 \text { therapy }\end{array}$ & $\begin{array}{l}\text { Individu } \\
\text { al } \\
\text { therapy }\end{array}$ & $\begin{array}{l}\text { Individ } \\
\text { ual } \\
\text { therapy }\end{array}$ \\
\hline \multicolumn{11}{|l|}{ Dysthymia } \\
\hline primary & $\begin{array}{l}\text { Individu } \\
\text { al } \\
\text { therapy }\end{array}$ & $\begin{array}{l}\text { Individua } \\
1 \text { therapy }\end{array}$ & $\begin{array}{l}\text { Individ } \\
\text { ual } \\
\text { therapy }\end{array}$ & $\begin{array}{l}\text { Pharmac } \\
\text { ology }\end{array}$ & $\begin{array}{l}\text { Pharma } \\
\text { cology }\end{array}$ & $\begin{array}{l}\text { Individu } \\
\text { al } \\
\text { therapy }\end{array}$ & $\begin{array}{l}\text { Pharma } \\
\text { cology }\end{array}$ & $\begin{array}{l}\text { Individua } \\
1 \text { therapy }\end{array}$ & $\begin{array}{l}\text { Individu } \\
\text { al } \\
\text { therapy }\end{array}$ & $\begin{array}{l}\text { Individ } \\
\text { ual } \\
\text { therapy }\end{array}$ \\
\hline secondary & $\begin{array}{l}\text { Group } \\
\text { therapy }\end{array}$ & $\begin{array}{l}\text { Pharmaco } \\
\text { logy }\end{array}$ & $\begin{array}{l}\text { Pharma } \\
\text { cology }\end{array}$ & $\begin{array}{l}\text { Individu } \\
\text { al } \\
\text { therapy }\end{array}$ & $\begin{array}{l}\text { Individ } \\
\text { ual } \\
\text { therapy }\end{array}$ & $\begin{array}{l}\text { Pharmac } \\
\text { ology }\end{array}$ & $\begin{array}{l}\text { Individ } \\
\text { ual } \\
\text { therapy }\end{array}$ & $\begin{array}{l}\text { Pharmaco } \\
\text { logy }\end{array}$ & $\begin{array}{l}\text { Pharmac } \\
\text { ology }\end{array}$ & $\begin{array}{l}\text { Pharma } \\
\text { cology }\end{array}$ \\
\hline
\end{tabular}

Typical treatment in enhanced specialty referral

Major

depressive

disorder 


\begin{tabular}{|c|c|c|c|c|c|c|c|c|c|c|}
\hline primary & $\begin{array}{l}\text { Individu } \\
\text { al } \\
\text { therapy }\end{array}$ & $\begin{array}{l}\text { Pharmaco } \\
\text { logy }\end{array}$ & $\begin{array}{l}\text { Pharma } \\
\text { cology }\end{array}$ & $\begin{array}{l}\text { Individu } \\
\text { al } \\
\text { therapy }\end{array}$ & $\begin{array}{l}\text { Pharma } \\
\text { cology }\end{array}$ & $\begin{array}{l}\text { Pharmac } \\
\text { ology }\end{array}$ & $\begin{array}{l}\text { Pharma } \\
\text { cology }\end{array}$ & $\begin{array}{l}\text { Pharmaco } \\
\text { logy }\end{array}$ & $\begin{array}{l}\text { Pharmac } \\
\text { ology }\end{array}$ & $\begin{array}{l}\text { Pharma } \\
\text { cology }\end{array}$ \\
\hline secondary & $\begin{array}{l}\text { Pharmac } \\
\text { ology }\end{array}$ & $\begin{array}{l}\text { Individua } \\
1 \text { therapy }\end{array}$ & $\begin{array}{l}\text { Individ } \\
\text { ual } \\
\text { therapy }\end{array}$ & $\begin{array}{l}\text { Pharmac } \\
\text { ology }\end{array}$ & $\begin{array}{l}\text { Individ } \\
\text { ual } \\
\text { therapy }\end{array}$ & $\begin{array}{l}\text { Individu } \\
\text { al } \\
\text { therapy }\end{array}$ & $\begin{array}{l}\text { Individ } \\
\text { ual } \\
\text { therapy }\end{array}$ & $\begin{array}{l}\text { Individua } \\
1 \text { therapy }\end{array}$ & $\begin{array}{l}\text { Individu } \\
\text { al } \\
\text { therapy }\end{array}$ & $\begin{array}{l}\text { Group } \\
\text { therapy }\end{array}$ \\
\hline \multicolumn{11}{|l|}{ Dysthymia } \\
\hline primary & $\begin{array}{l}\text { Individu } \\
\text { al } \\
\text { therapy }\end{array}$ & $\begin{array}{l}\text { Individua } \\
1 \text { therapy }\end{array}$ & $\begin{array}{l}\text { Individ } \\
\text { ual } \\
\text { therapy }\end{array}$ & $\begin{array}{l}\text { Individu } \\
\text { al } \\
\text { therapy }\end{array}$ & $\begin{array}{l}\text { Pharma } \\
\text { cology }\end{array}$ & $\begin{array}{l}\text { Pharmac } \\
\text { ology }\end{array}$ & $\begin{array}{l}\text { Pharma } \\
\text { cology }\end{array}$ & $\begin{array}{l}\text { Pharmaco } \\
\text { logy }\end{array}$ & $\begin{array}{l}\text { Individu } \\
\text { al } \\
\text { therapy }\end{array}$ & $\begin{array}{l}\text { Pharma } \\
\text { cology }\end{array}$ \\
\hline secondary & $\begin{array}{l}\text { Pharmac } \\
\text { ology }\end{array}$ & $\begin{array}{l}\text { Pharmaco } \\
\text { logy }\end{array}$ & $\begin{array}{l}\text { Pharma } \\
\text { cology }\end{array}$ & $\begin{array}{l}\text { Pharmac } \\
\text { ology }\end{array}$ & $\begin{array}{l}\text { Individ } \\
\text { ual } \\
\text { therapy }\end{array}$ & $\begin{array}{l}\text { Individu } \\
\text { al } \\
\text { therapy }\end{array}$ & $\begin{array}{l}\text { Individ } \\
\text { ual } \\
\text { therapy }\end{array}$ & $\begin{array}{l}\text { Individua } \\
1 \text { therapy }\end{array}$ & $\begin{array}{l}\text { Pharmac } \\
\text { ology }\end{array}$ & $\begin{array}{l}\text { Group } \\
\text { therapy }\end{array}$ \\
\hline
\end{tabular}


Table 3.4

Baseline Characteristics of Participants in the Primary Care Research in Substance Abuse and Mental Health for the Elderly Study $(N=585)$

\begin{tabular}{|c|c|c|c|c|c|c|}
\hline \multirow[b]{2}{*}{ Sample characteristics } & \multicolumn{2}{|c|}{$\begin{array}{l}\text { Both groups } \\
\quad(N=585)\end{array}$} & \multicolumn{2}{|c|}{$\begin{array}{l}\text { Integrated Care } \\
\quad(N=296) \\
\end{array}$} & \multicolumn{2}{|c|}{$\begin{array}{l}\text { Enhanced Specialty Referral } \\
\qquad(N=289)\end{array}$} \\
\hline & $N$ & $\%$ & $N$ & $\%$ & $N$ & $\%$ \\
\hline Gender & $N=577$ & & $N=291$ & & $N=286$ & \\
\hline Female & 377 & 65.3 & 185 & 63.6 & 192 & 63.5 \\
\hline \multicolumn{7}{|l|}{ Age } \\
\hline Age (mean \pm SD years) & $73 \pm 6.5$ & & $72.8 \pm 6.4$ & & $73.1 \pm 6.6$ & \\
\hline Race & $N=583$ & & $N=295$ & & $N=288$ & \\
\hline White & 215 & 36.8 & 117 & 39.6 & 98 & 34 \\
\hline African-American & 100 & 17.1 & 45 & 15.2 & 55 & 19 \\
\hline Hispanic/Latino & 169 & 28.9 & 84 & 28.4 & 85 & 29.5 \\
\hline Asian & 79 & 13.5 & 33 & 11.1 & 46 & 15.9 \\
\hline Other & 20 & 3.4 & 16 & 5.4 & 4 & 1.3 \\
\hline Martial Status & $N=583$ & & $N=296$ & & $N=\mathbf{2 8 7}$ & \\
\hline Married & 211 & 36.1 & 109 & 36.8 & 102 & 35.5 \\
\hline Separated & 35 & 6 & 19 & 6.4 & 16 & 5.5 \\
\hline Divorced & 91 & 15.6 & 42 & 14.1 & 49 & 17 \\
\hline Widowed & 208 & 35.6 & 102 & 34.4 & 106 & 36.9 \\
\hline Never married & 38 & 6.5 & 24 & 8.1 & 14 & 4.8 \\
\hline Education & $N=582$ & & $N=295$ & & $N=\mathbf{2 8 7}$ & \\
\hline Less than 8 th grade & 209 & 35.7 & 98 & 33.2 & 111 & 38.6 \\
\hline Less than 12 th grade & 93 & 15.8 & 47 & 15.9 & 46 & 16 \\
\hline
\end{tabular}


High school

graduate/GED

Some college or trade

school

College graduate

Graduate school

Financial Status

Limited finances

Behavioral Health

Issues

At-risk drinking

Suicidal ideation

Death ideation

Past year Anxiety

Anxiety NOS

Generalized Anxiety

Disorder

Panic Disorder

Past Year

Depression

Major depressive

disorder

Subthreshold

depression

No depression
15.5

14.3

9.7

8.2

32.4

186

$N=584$

58

62

186

$N=585$

71

12.1

4.1

24

6

9.9

10.6

31.8

$N=585$

309

52.8

183

31.2

15.8
17.9

15.2

9.1

8.4

29.1

$N=292$

85

$N=295$

$$
27
$$

26

101

N

$N=296$

37

5

8.8

34.2

11

5

$N$
$N=296$

160

53

91

30.7
13.2

13.5

10.4

8

35.8

10.7

12.4

29.4

$N=289$

$34 \quad 11.7$

$13 \quad 4.4$

$1 \quad 0.3$

$N=\mathbf{2 8 9}$

$149 \quad 51.5$

$92 \quad 31.8$

$48 \quad 16.6$ 
Table 3.5

Baseline Characteristics of Variables of Interest of Participants in the Primary Care Research in Substance Abuse and Mental Health for the Elderly Study $(N=585)$

\begin{tabular}{|c|c|c|c|c|c|c|c|c|c|c|}
\hline & \multicolumn{2}{|c|}{$\begin{array}{l}\text { San Francisco, } \\
\text { California } \\
(N=153)\end{array}$} & \multicolumn{2}{|c|}{$\begin{array}{c}\text { Chinatown, New } \\
\text { York } \\
(N=175)\end{array}$} & \multicolumn{2}{|c|}{$\begin{array}{l}\text { Sunset Park, New } \\
\text { York } \\
(N=75)\end{array}$} & \multicolumn{2}{|c|}{$\begin{array}{l}\text { Rochester, New } \\
\text { York } \\
\quad(N=84)\end{array}$} & \multicolumn{2}{|c|}{$\begin{array}{l}\text { Philadelphia, } \\
\text { Pennsylvania } \\
\qquad(N=98)\end{array}$} \\
\hline & $n$ & $\%$ & $\mathrm{n}$ & $\%$ & $\mathrm{n}$ & $\%$ & $\mathrm{n}$ & $\%$ & $\mathrm{n}$ & $\%$ \\
\hline Gender & $N=152$ & & $N=175$ & & $N=69$ & & $N=83$ & & $N=98$ & \\
\hline Female & 93 & 61.1 & 132 & 75.4 & 39 & 56.5 & 55 & 66.2 & 58 & 59.5 \\
\hline Male & 59 & 38.8 & 43 & 24.5 & 30 & 43.4 & 28 & 33.7 & 40 & 40.4 \\
\hline Race & $N=152$ & & $N=175$ & & $N=74$ & & $N=84$ & & $N=98$ & \\
\hline White & 60 & 39.4 & 9 & 5.1 & 0 & 0 & 78 & 92.8 & 68 & 69.3 \\
\hline African-American & 68 & 44.7 & 0 & 0 & 0 & 0 & 4 & 4.7 & 28 & 28.5 \\
\hline Asian & 3 & 1.9 & 2 & 1.1 & 73 & 98.6 & 0 & 0 & 1 & 1 \\
\hline Hispanic/Latino & 5 & 3.2 & 161 & 92 & 1 & 1.3 & 2 & 2.3 & 0 & 0 \\
\hline Other & 16 & 10.5 & 3 & 1.7 & 0 & 0 & 0 & 0 & 1 & 1 \\
\hline Life stressors & $N=153$ & & $N=175$ & & $N=75$ & & $N=84$ & & $N=98$ & \\
\hline Loss of family/friends & 78 & 50.9 & 83 & 47.4 & 13 & 17.3 & 64 & 76.1 & 18 & 18.3 \\
\hline Lack of activities/work & 55 & 35.9 & 49 & 28 & 8 & 10.6 & 44 & 52.3 & 6 & 6.1 \\
\hline Family issues & 70 & 45.7 & 79 & 45.1 & 38 & 50.6 & 52 & 61.9 & 14 & 14.2 \\
\hline Money issues & 55 & 35.9 & 42 & 24 & 30 & 40 & 44 & 52.3 & 9 & 9.1 \\
\hline Medical illness & 67 & 43.7 & 126 & 72 & 43 & 57.3 & 66 & 78.5 & 19 & 19.3 \\
\hline Moving/relocation & 37 & 24.1 & 92 & 52.5 & 6 & 8 & 48 & 57.1 & 0 & 0 \\
\hline
\end{tabular}




\begin{tabular}{|c|c|c|c|c|c|c|c|c|c|c|}
\hline $\begin{array}{l}\text { Chronic medical } \\
\text { conditions }\end{array}$ & $N=151$ & & $N=175$ & & $N=71$ & & $N=84$ & & $N=94$ & \\
\hline $\begin{array}{l}\text { Diabetes or sugar } \\
\text { sickness }\end{array}$ & 117 & 77.4 & 108 & 61.7 & 56 & 78.8 & 66 & 78.5 & 81 & 86.1 \\
\hline $\begin{array}{l}\text { High blood pressure or } \\
\text { Hypertension }\end{array}$ & 87 & 57.6 & 121 & 69.1 & 38 & 53.5 & 52 & 61.9 & 55 & 58.5 \\
\hline Heart trouble & 45 & 29.8 & 43 & 24.5 & 22 & 30.9 & 37 & 44 & 30 & 31.9 \\
\hline Broken hip & 3 & 1.9 & 3 & 1.7 & 4 & 5.6 & 2 & 2.3 & 2 & 2.1 \\
\hline \multicolumn{11}{|l|}{$\begin{array}{l}\text { Behavioral health } \\
\text { conditions }\end{array}$} \\
\hline & $N=153$ & & $N=175$ & & $N=74$ & & $N=84$ & & $N=98$ & \\
\hline At-risk drinking & $\begin{array}{l}24 \\
N=153\end{array}$ & 15.6 & $\begin{array}{l}7 \\
N=175\end{array}$ & 4 & $\begin{array}{l}0 \\
N=74\end{array}$ & 0 & $\begin{array}{l}13 \\
N=84\end{array}$ & 15.4 & $\begin{array}{l}14 \\
N=98\end{array}$ & 14.1 \\
\hline Suicidal ideation & 19 & 12.4 & 7 & 4 & 15 & 20.2 & 11 & 13 & 10 & 10.2 \\
\hline Death ideation & $\begin{array}{l}41 \\
N=153\end{array}$ & 26.7 & $\begin{array}{l}59 \\
N=175\end{array}$ & 33.7 & $\begin{array}{l}29 \\
N=75\end{array}$ & 39.1 & $\begin{array}{l}22 \\
N=84\end{array}$ & 26.1 & $\begin{array}{l}35 \\
N=98\end{array}$ & 35.7 \\
\hline Anxiety NOS & 33 & 21.5 & 21 & 12 & 7 & 9.3 & 3 & 3.5 & 7 & 7.1 \\
\hline $\begin{array}{l}\text { Generalized Anxiety } \\
\text { Disorder }\end{array}$ & 4 & 2.6 & 6 & 3.4 & 1 & 1.3 & 5 & 5.9 & 8 & 8.1 \\
\hline Panic Disorder & $\begin{array}{l}2 \\
N=153\end{array}$ & 1.3 & $\begin{array}{l}2 \\
N=175\end{array}$ & 1.1 & $\begin{array}{l}0 \\
N=75\end{array}$ & 0 & $\begin{array}{l}2 \\
N=84\end{array}$ & 2.3 & $\begin{array}{l}0 \\
N=98\end{array}$ & 0 \\
\hline $\begin{array}{l}\text { Major depressive } \\
\text { disorder } \\
\text { Subthreshold }\end{array}$ & 55 & 35.9 & 142 & 81.1 & 63 & 84 & 19 & 22.6 & 30 & 30.6 \\
\hline depression & 68 & 44.4 & 26 & 14.9 & 7 & 9.3 & 40 & 47.6 & 42 & 42.9 \\
\hline No depression & 30 & 19.6 & 7 & 4 & 5 & 6.7 & 25 & 29.8 & 26 & 26.5 \\
\hline
\end{tabular}


Table 4.1

Changes in Depression Severity From Baseline, Three-Month, and Six-Month Follow-Up $(N=577)$

\begin{tabular}{|c|c|c|c|c|c|c|c|c|c|}
\hline & \multicolumn{3}{|c|}{ Baseline } & \multicolumn{4}{|c|}{ Three-month } & \multicolumn{2}{|c|}{$\underline{\text { Six-month }}$} \\
\hline & $N$ & Mean & $S D$ & $N$ & Mean & $S D$ & $N$ & $\overline{\text { Mean }}$ & $S D$ \\
\hline \multicolumn{10}{|c|}{ Enhanced Specialty } \\
\hline \multicolumn{10}{|c|}{ Referral } \\
\hline Male & 94 & 24.26 & 11.86 & 94 & 20.31 & 11.37 & 94 & 18.01 & 10.50 \\
\hline Female & 192 & 24.48 & 11.63 & 192 & 21.44 & 11.22 & 192 & 19.62 & 11.26 \\
\hline \multicolumn{10}{|c|}{ Integrated Care } \\
\hline Male & 106 & 24.11 & 11.10 & 106 & 21.77 & 10.00 & 106 & 19.92 & 10.63 \\
\hline Female & 185 & 25.37 & 10.39 & 185 & 23.21 & 11.30 & 185 & 21.77 & 11.86 \\
\hline
\end{tabular}

Note: This analysis excludes eight participants that reported "don't know/unknown" when asked their gender. 
Table 4.2

Correlation Matrix of Variables in the Enhanced Specialty Referral Model ( $N=867)$

\begin{tabular}{|c|c|c|c|c|c|c|c|c|c|c|c|c|c|c|c|c|c|c|c|}
\hline Variable & 1 & 2 & 3 & 4 & 5 & 6 & 7 & 8 & 9 & 10 & 11 & 12 & 13 & 14 & 15 & 16 & 17 & 18 & 19 \\
\hline 1. Depression severity & 1 & & & & & & & & & & & & & & & & & & \\
\hline 2. Study site & -0.03 & 1 & & & & & & & & & & & & & & & & & \\
\hline 3. Gender & 0.04 & -0.065 & 1 & & & & & & & & & & & & & & & & \\
\hline 4. Loss of family and friends & $-.079^{*}$ & $-.107 * *$ & 0.051 & 1 & & & & & & & & & & & & & & & \\
\hline 5. Lack of activities & -0.035 & $-.135 * *$ & 0.017 & $.297 * *$ & 1 & & & & & & & & & & & & & & \\
\hline 6. Family issues & 0.061 & $-.168^{* *}$ & $.095 * *$ & $.191^{*}$ & $.179 * *$ & & & & & & & & & & & & & & \\
\hline 7. Money issues & 0.067 & $-.084^{*}$ & 0.043 & $.263 * *$ & $.329 * *$ & $390 * *$ & 1 & & & & & & & & & & & & \\
\hline 8.Medical illness & $.178 * *$ & $-.120 * *$ & -0.021 & $.220 * *$ & $.292 * *$ & $.176 * *$ & $.186^{* *}$ & 1 & & & & & & & & & & & \\
\hline 9.Moving/relocation & $.100 *$ & $-.108^{* *}$ & -0.023 & $.286 * *$ & $.346 * *$ & $.167^{*}$ & $.174 * *$ & $.417 * *$ & 1 & & & & & & & & & & \\
\hline 10. Diabetes & $-.101 * *$ & 0.026 & -0.053 & -0.053 & $-.085^{*}$ & -0.042 & $-.087^{*}$ & $-.131 * *$ & $-.099 * *$ & 1 & & & & & & & & & \\
\hline 11. High Blood pressure & $-.067^{*}$ & -0.008 & $-.133 * *$ & $-.127^{* *}$ & -0.02 & -0.037 & $-.079 *$ & $-.150^{* *}$ & -0.057 & $.651 *$ & 1 & & & & & & & & \\
\hline 12. Heart trouble & -0.019 & -0.005 & -0.041 & $-.154 * *$ & -0.06 & -0.048 & $-.087^{*}$ & $-.156^{* *}$ & -0.047 & $.611^{* *}$ & $.783 * *$ & 1 & & & & & & & \\
\hline 13. Broken hip & -0.019 & 0.03 & -0.042 & $-.144 * *$ & $-.071^{*}$ & $-.091 * *$ & -0.066 & $-.126^{* *}$ & $-.084^{*}$ & $.492 * *$ & $.652 * *$ & $.723 *$ & 1 & & & & & & \\
\hline 14. Time & $-.188 * *$ & 0 & 0 & 0 & 0 & 0 & 0 & 0 & 0 & 0 & 0 & 0 & 0 & 0 & 1 & & & & \\
\hline 15.Paykel suicide score & $.329 * *$ & 0.004 & -0.013 & 0.024 & 0.032 & 0.007 & $.085^{*}$ & 0.05 & $-.079^{*}$ & 0.002 & 0 & -0.034 & -0.044 & c & $-.158 * *$ & 1 & & & \\
\hline 16. Severity of life stress & $.109 * *$ & $-.208 * *$ & $.105 *$ & $.658 * *$ & $.500 * *$ & $.621 \cdots$ & $.489 * *$ & $.625 * *$ & $.462 *$ & $-.143 * *$ & $-.173 * *$ & $-.175 * *$ & $-.164 * *$ & c & 0 & 0.038 & 1 & & \\
\hline 17. Depression type & $-.226 * *$ & $.142 * *$ & $-.099 * *$ & 0.028 & 0.001 & 0.014 & -0.034 & 0.019 & 0.034 & 0.031 & 0.053 & 0.018 & 0.022 & c & $.271^{*}$ & $-.159 * *$ & -0.001 & 1 & \\
\hline 18. Alcohol Consumption & -0.052 & 0.038 & $-.193 * *$ & 0.02 & $-.080^{*}$ & $-.082^{*}$ & $-.085^{*}$ & $-.070^{*}$ & 0.002 & 0.009 & -0.031 & -0.05 & $-.076^{*}$ & $c$ & $-.096 * *$ & -0.013 & $-.104 * *$ & $.186 * *$ & 1 \\
\hline 19. Anxiety type & $.198 * *$ & -0.062 & $.125 * *$ & 0.033 & $.077^{*}$ & $.079^{*}$ & $.104 * *$ & 0.043 & 0.048 & -0.041 & -0.023 & -0.016 & 0.016 & $c$ & $-.080^{*}$ & $.163^{* *}$ & $.079^{*}$ & -0.055 & \\
\hline
\end{tabular}

- Correlation is significant at the 0.05 level ( 2 -tailed).

* Correlation is significant at the 0.01 level (2-tailed).

c Cannot be computed because at least one of the variables is constant. 


\section{Table 4.3}

Collinearity Statistics for Enhanced Specialty Referral Model $(N=867)$

\begin{tabular}{|c|c|c|}
\hline \multirow[b]{2}{*}{ Variable } & \multicolumn{2}{|c|}{ Collinearity Diagnostics } \\
\hline & Tolerance & VIF \\
\hline Gender & 0.892 & 1.121 \\
\hline Severity of life stress* & 0.091 & 10.994 \\
\hline Loss of family and friends & 0.321 & 3.116 \\
\hline Lack of activities & 0.642 & 1.557 \\
\hline Family issues & 0.332 & 3.008 \\
\hline Money issues & 0.7 & 1.429 \\
\hline Medical illness & 0.347 & 2.878 \\
\hline Moving/relocation & 0.709 & 1.41 \\
\hline Diabetes & 0.534 & 1.872 \\
\hline High Blood pressure & 0.311 & 3.22 \\
\hline Heart trouble & 0.291 & 3.433 \\
\hline Broken hip & 0.444 & 2.252 \\
\hline Time & 0.885 & 1.129 \\
\hline Paykel suicide score & 0.909 & 1.1 \\
\hline Depression type & 0.835 & 1.198 \\
\hline Alcohol Consumption & 0.877 & 1.14 \\
\hline Anxiety type & 0.933 & 1.071 \\
\hline
\end{tabular}




\section{Table 4.4}

Correlation Matrix of Variables in the Integrated Care Model $(N=888)$

\begin{tabular}{|c|c|c|c|c|c|c|c|c|c|c|c|c|c|c|c|c|c|c|c|}
\hline Variable & 1 & 2 & 3 & 4 & 5 & 6 & 7 & 8 & 9 & 10 & 11 & 12 & 13 & 14 & 15 & 16 & 17 & 18 & 19 \\
\hline $\begin{array}{l}\text { 1. Depression severity } \\
\text { 2. Study site }\end{array}$ & $\begin{array}{l}1 \\
-0.025\end{array}$ & 1 & & & & & & & & & & & & & & & & & \\
\hline 3. Gender & 0.066 & -0.017 & 1 & & & & & & & & & & & & & & & & \\
\hline 4. Loss of family and friends & $-.102 * *$ & $-.143 * *$ & $.086^{*}$ & 1 & & & & & & & & & & & & & & & \\
\hline 5. Lack of activities & 0.008 & $-.134 * *$ & 0.018 & $.368 * \bullet$ & 1 & & & & & & & & & & & & & & \\
\hline 6. Family issues & 0.003 & $-.079^{*}$ & 0.066 & $.262^{* *}$ & $.324 * *$ & 1 & & & & & & & & & & & & & \\
\hline 7. Money issues & -0.023 & $-.079^{*}$ & -0.01 & $.383^{* *}$ & $.408 * *$ & $.522 * *$ & 1 & & & & & & & & & & & & \\
\hline 8.Medical illness & $.121 * *$ & $-.100 * *$ & 0.021 & $.295 * *$ & $.379 * *$ & $.286^{\circ *}$ & $.275^{* *}-x-1$ & 1 & & & & & & & & & & & \\
\hline 9.Moving/relocation & $.076^{*}$ & $-.190^{* * *}$ & $.137 * *$ & $.396 * *$ & $.423 * *$ & $.211^{* *}$ & $.235 * *$ & $.440 * *$ & 1 & & & & & & & & & & \\
\hline 10. Diabetes & 0 & $.179 * *$ & -0.01 & -0.051 & $-.113^{* *}$ & -0.018 & -0.01 & $-.108 * *$ & $-.145^{* *}$ & 1 & & & & & & & & & \\
\hline 11. High Blood pressure & 0.013 & $.148 * *$ & $-.068^{*}$ & $-.086^{*}$ & $-.118 * *$ & -0.017 & -0.05 & $-.141 * *$ & $-.156 * *$ & $.670 * *$ & & & & & & & & & \\
\hline 12. Heart trouble & 0.03 & $.080^{*}$ & -0.06 & $-.069^{*}$ & $-.077^{*}$ & -0.017 & -0.06 & $-.169 * *$ & $-.088 * *$ & $.462^{* *}$ & $.678^{* *}-x$ & 1 & & & & & & & \\
\hline 13. Broken hip & $.069^{*}$ & $.122 * *$ & -0.05 & $-.118 * *$ & $-.119 * *$ & -0.002 & 0.066 & -0.018 & $-.118 * *$ & $.704 * *$ & $.709 * *$ & $.544 * *$ & 1 & c. & & & & & \\
\hline 14. Time & $-.140^{* *}$ & 0 & 0 & 0 & 0 & 0 & 0 & 0 & 0 & 0 & 0 & 0 & 0 & c & 1 & & & & \\
\hline 15.Paykel suicide score & $.297 * *$ & 0.017 & 0.006 & -0.061 & -0.046 & -0.012 & $.071^{*}$ & -0.032 & -0.01 & 0.022 & 0.031 & 0.006 & $.078^{*}$ & c & $-.126 * *$ & 1 & & & \\
\hline 16. Severity of life stress & 0.061 & $-.208 * *$ & 0.057 & $.707 * *$ & $.623 * *$ & $.644^{* *}$ & $.574 * *$ & $.660 * *$ & $551^{* *}$ & $-.118 * 4$ & $-.119 * 4$ & $-.097 * *$ & $-.075^{*}$ & c & 0 & -0.039 & 1 & & \\
\hline 17. Depression type & $-.202 * *$ & 0.024 & $-.086^{*}$ & 0.025 & 0.002 & 0.016 & -0.01 & 0.061 & -0.013 & 0.039 & 0.016 & -0.008 & -0.03 & c. & $.204 * *$ & $-.148 * *$ & 0.014 & 1 & \\
\hline 18. Alcohol Consumption & $-.070^{*}$ & $.177^{* *}$ & $-.254 * *$ & 0.012 & -0.042 & -0.032 & -0.03 & 0.034 & $-.158 * *$ & 0.023 & 0.018 & -0.007 & $-.071^{*}$ & c & -0.042 & $-.143^{* *}$ & -0.03 & $.293 * *$ & \\
\hline 19. Anxiety type & $.187^{* *}$ & -0.033 & $.095 * *$ & $.101^{* *}$ & $.106 * *$ & $.106 * *$ & $.087 * *$ & 0.052 & $.175 * *$ & $.156^{* *}$ & $.075^{*}$ & 0.047 & $.092 * *$ & c. & $-.107^{* *}$ & $.128 * *$ & $.101 * *$ & $-.095 * *$ & $-.122 * *$ \\
\hline
\end{tabular}

ificant at the 0.05 level (2-tailed).

c Cannot be computed because at least one of the variables is constant. 
Table 4.5

Collinearity Statistics for Integrated Care Model $(N=888)$

\begin{tabular}{lrr} 
& \multicolumn{2}{c}{ Collinearity Diagnostics } \\
\cline { 2 - 3 } Variable & 0.895 & 1.118 \\
& 0.086 & 11.598 \\
Gender & & \\
Severity of life stress* & 0.302 & 3.306 \\
Loss of family and & 0.53 & 1.888 \\
friends & 0.356 & 2.81 \\
Lack of activities & 0.574 & 1.743 \\
Family issues & 0.376 & 2.657 \\
Money issues & 0.598 & 1.672 \\
Medical illness & 0.414 & 2.416 \\
Moving/relocation & 0.33 & 3.031 \\
Diabetes & 0.513 & 1.948 \\
High Blood pressure & 0.349 & 2.865 \\
Heart trouble & 0.927 & 1.079 \\
Broken hip & 0.922 & 1.085 \\
Time & 0.849 & 1.178 \\
Paykel suicide score & 0.771 & 1.296 \\
Depression type & 0.882 & 1.134 \\
Alcohol Consumption &
\end{tabular}

Dependent Variable: Depression severity as measured by the CES-D (Radloff, 1997).

*Unacceptable tolerance and VIF value. 
Table 4.6

Akaike's Information Criterion (AIC) and Bayesian Information Criterion (BIC) For Each Hierarchical Linear Model

AIC

Randominter

3826.35

$\mathrm{BIC}$

Random intercept-only model; subthreshold depression subset

2704.88 3909.11

Random intercept-only model; major depressive disorder subse

5419.35

Random intercept model with level-2 predictors (i.e., frequency of life stressors); no depression diagnosis subset

$3817.13 \quad 3978.3$

Random intercept model with level-2 predictors (i.e., frequency of life stressors); subthreshold depression diagnosis subset 2727.24

2875.66

Random intercept model with level-2 predictors (i.e., frequency of life stressors); major depressive disorder subset

Random intercept model with level-2 predictors (i.e.., gender); no depression subset

Random intercept model with level-2 predictors (i.e..., gender); subthreshold depression subset

5348.2

$3831.07 \quad 3926.91$

Random intercept model with level-2 predictors (i.e.., gender); major depressive disorder

$5329.87 \quad 5431.42$

Random intercept model with level-2 predictors (i.e., interaction term of life stressors and gender); no depression diagnosis subset

$3843.95 \quad 4096.6$ Random intercept model with level-2 predictors (i.e., interaction term of life stressors and gender); Subthreshold diagnosis subset

$\begin{array}{rr}2737.3 & 2969.96 \\ 5359.35 & 5627.08\end{array}$

Random intercept model with level-2 predictors (i.e., interaction term of life stressors and gender); major depressive disorder diagnosis subset

5359.35

Dependent Variable: Depression severity as measured by the CES.D (Radloff, 1997).

"Lowest AIC or BIC scores indicates best model fit 
Table 4.7

Differences in Average Scores on the CES-D Among Participants Stratified Across Levels of Depression (N=585)

\begin{tabular}{|c|c|c|c|c|c|c|}
\hline & Variable & Estimate & $\mathrm{SE}$ & $\mathrm{df}$ & $t$ & Sig. \\
\hline \multirow[t]{11}{*}{ Major depressive disorder } & Intercept & 26.275104 & 2.121456 & 571.509 & 12.385 & 0.00 \\
\hline & Diabetes & -0.454489 & 0.452382 & 320.217 & -1.005 & 0.32 \\
\hline & High blood pressure & -0.648343 & 0.520395 & 345.801 & -1.246 & 0.21 \\
\hline & Heart trouble & 0.057223 & 0.476636 & 403.95 & 0.12 & 0.90 \\
\hline & Suicidal ideation** & 4.454597 & 1.205837 & 719.826 & 3.694 & 0.00 \\
\hline & Death ideation $* *$ & 3.164299 & 0.665841 & 707.339 & 4.752 & 0.00 \\
\hline & $\begin{array}{l}\text { No suicidal ideation or death } \\
\text { ideation }\end{array}$ & . & . & . & · & . \\
\hline & Anxiety NOS** & 3.391138 & 0.845088 & 696.096 & 4.013 & 0.00 \\
\hline & Generalized anxiety disorder & -0.103319 & 1.721939 & 746.652 & -0.06 & 0.95 \\
\hline & Panic disorder** & 4.997712 & 2.266082 & 745.565 & 2.205 & 0.03 \\
\hline & No anxiety diagnosis & . & . & . & . & . \\
\hline
\end{tabular}




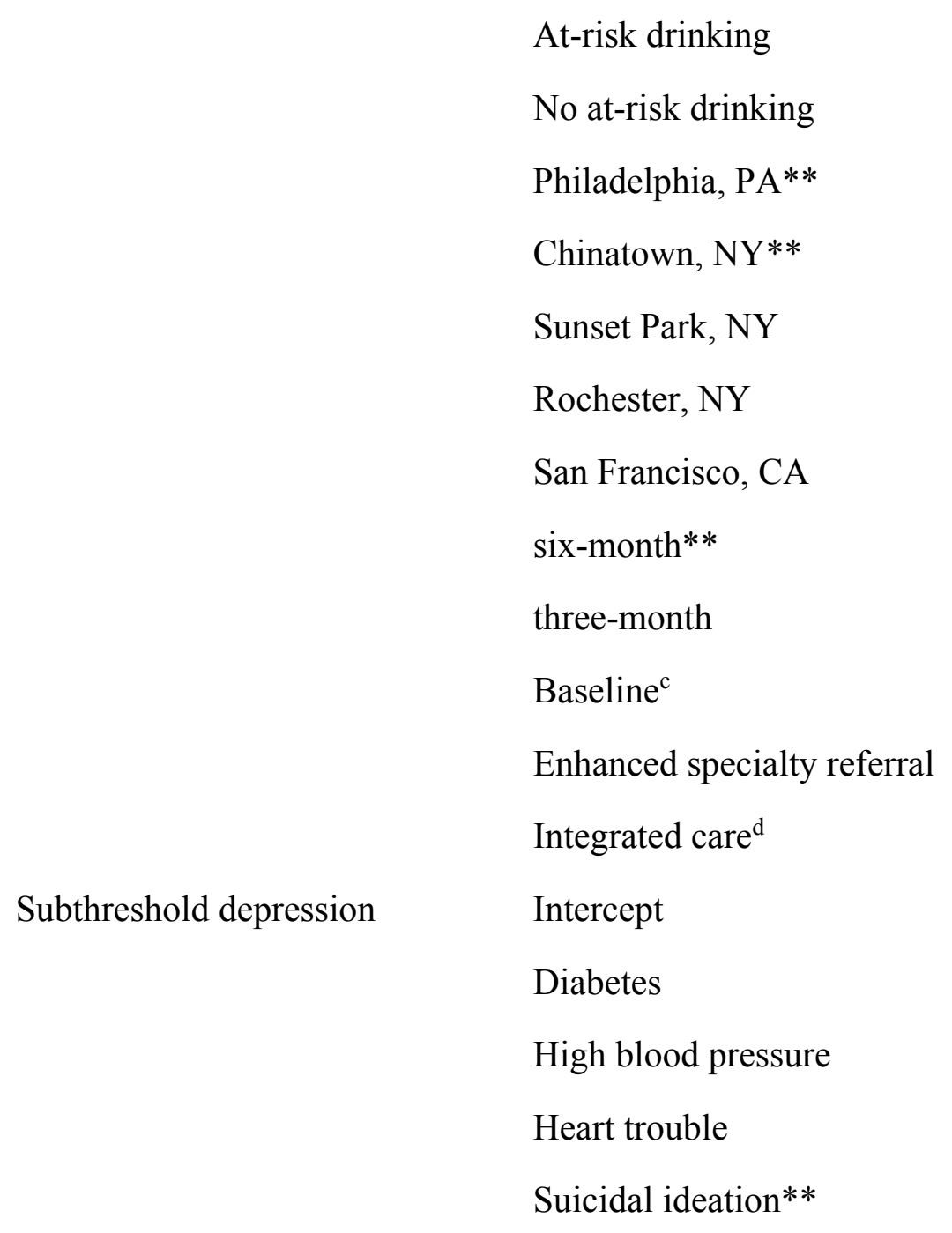

\begin{tabular}{|c|c|c|c|c|}
\hline-1.993065 & 1.785746 & 650.258 & -1.116 & 0.27 \\
\hline & & . & & \\
\hline 3.558557 & 1.55038 & 422.507 & 2.295 & 0.02 \\
\hline 6.849912 & 1.094718 & 381.986 & 6.257 & 0.00 \\
\hline 11.885032 & 1.331966 & 355.782 & 8.923 & 0.00 \\
\hline 0.147469 & 1.708898 & 428.525 & 0.086 & 0.93 \\
\hline & & . & . & \\
\hline-1.576809 & 0.667494 & 498.409 & -2.362 & 0.02 \\
\hline-0.720372 & 0.630728 & 486.663 & -1.142 & 0.25 \\
\hline & & . & . & • \\
\hline-0.715571 & 0.80095 & 350.678 & -0.893 & 0.37 \\
\hline & & . & . & . \\
\hline 16.422734 & 1.557342 & 382.83 & 10.545 & 0.00 \\
\hline-0.52342 & 0.597751 & 301.986 & -0.876 & 0.38 \\
\hline-0.47986 & 0.560336 & 289.314 & -0.856 & 0.39 \\
\hline 0.540488 & 0.447237 & 272.137 & 1.209 & 0.23 \\
\hline 4.434098 & 1.370299 & 387.501 & 3.236 & 0.00 \\
\hline
\end{tabular}


Death ideation

No suicidal ideation or death ideation

Anxiety NOS**

Generalized anxiety disorder**

Panic disorder**

No anxiety diagnosis

At-risk drinking

No at-risk drinking

Philadelphia, PA

Chinatown, $\mathrm{NY}^{* *}$

Sunset Park, NY**

Rochester, NY

San Francisco, CA

six-month**

three-month

Baseline $^{\mathrm{c}}$ $\begin{array}{lllll}2.871625 & 0.780806 & 405.678 & 3.678 & 0.00\end{array}$

\begin{tabular}{|c|c|c|c|c|}
\hline 5.224176 & 1.014524 & 407.222 & 5.149 & 0.00 \\
\hline 5.906116 & 1.556538 & 334.504 & 3.794 & 0.00 \\
\hline 12.190098 & 3.292634 & 406.234 & 3.702 & 0.00 \\
\hline & & . & • & \\
\hline-0.351483 & 1.251998 & 407.555 & -0.281 & 0.7 \\
\hline & & . & & \\
\hline 1.903316 & 1.018789 & 252.818 & 1.868 & 0.06 \\
\hline 2.266629 & 1.042733 & 296.617 & 2.174 & 0.03 \\
\hline 4.688822 & 1.594539 & 261.206 & 2.941 & 0.00 \\
\hline-0.132948 & 1.054379 & 262.636 & -0.126 & 0.5 \\
\hline & & . & . & \\
\hline 1.350321 & 0.726015 & 286.966 & 1.86 & 0.06 \\
\hline 0.392484 & 0.694983 & 266.035 & 0.565 & 0.5 \\
\hline
\end{tabular}




\begin{tabular}{|c|c|c|c|c|c|c|}
\hline & Enhanced specialty referral & -0.987626 & 0.733517 & 271.778 & -1.346 & 0.18 \\
\hline & Integrated care ${ }^{\mathrm{d}}$ & . & & . & . & \\
\hline \multirow[t]{14}{*}{ No depression } & Intercept & 13.572032 & 1.273768 & 384.981 & 10.655 & 0.00 \\
\hline & Diabetes & -0.179923 & 0.743576 & 266.664 & -0.242 & 0.81 \\
\hline & High blood pressure & 0.533039 & 0.622549 & 255.684 & 0.856 & 0.39 \\
\hline & Heart trouble & -0.425669 & 0.576101 & 306.147 & -0.739 & 0.46 \\
\hline & Suicidal ideation & 0.606787 & 1.469924 & 411.832 & 0.413 & 0.68 \\
\hline & Death ideation & -1.108094 & 0.91067 & 529.652 & -1.217 & 0.22 \\
\hline & $\begin{array}{l}\text { No suicidal ideation or death } \\
\text { ideation }\end{array}$ & . & & . & . & \\
\hline & Anxiety NOS** & 3.976923 & 1.135914 & 543.282 & 3.501 & 0.00 \\
\hline & Generalized anxiety disorder** & 5.482164 & 1.612828 & 549.791 & 3.399 & 0.00 \\
\hline & Panic disorder & 10.400076 & 5.887878 & 290.87 & 1.766 & 0.08 \\
\hline & No anxiety diagnosis & $\cdot$ & & & 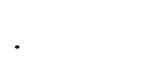 & \\
\hline & At-risk drinking $* *$ & -5.352145 & 0.81853 & 571.83 & -6.539 & 0.00 \\
\hline & No at-risk drinking & $\cdot$ & & . & - & \\
\hline & Philadelphia, PA & 0.819297 & 0.992965 & 223.983 & 0.825 & 0.41 \\
\hline
\end{tabular}




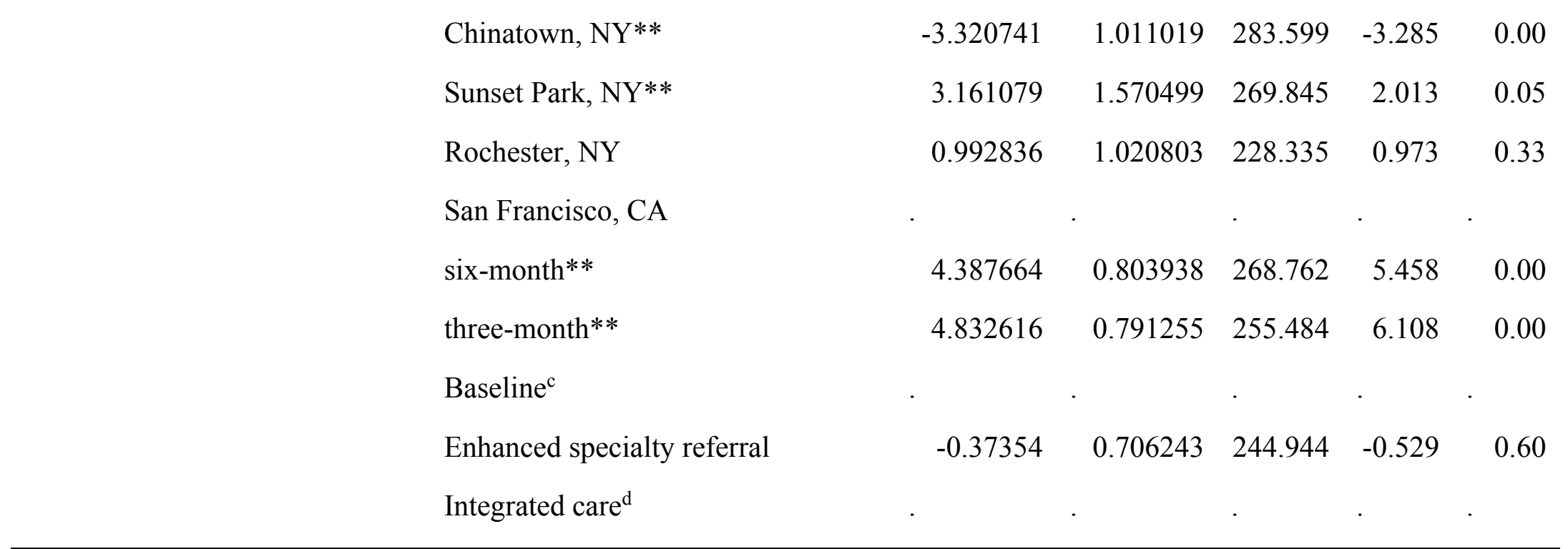

Dependent Variable: Depression severity as measured by the CES-D

(Radloff, 1997).

a The reference group category is: No depression.

${ }^{\mathrm{b}}$ The reference group category is: San Francisco, CA.

${ }^{\mathrm{c}}$ The reference group category

is: Baseline.

${ }^{\mathrm{d}}$ The reference group category is: Integrated Care

$* * p<.05$ 
Table 4.8

Impact of The Frequency of Life Stressors on Baseline, Three-Month, and Six Month Scores on the CES$D$ Among Participants Stratified Across Levels of Depression $(N=585)$

\begin{tabular}{|c|c|c|c|c|c|c|}
\hline & Variable & Estimate & SE & $\mathrm{df}$ & $t$ & Sig. \\
\hline & & & & & 11.30 & \\
\hline \multirow{9}{*}{ Major depressive disorder } & Intercept & 25.679667 & 2.272276 & 572.272 & 1 & 0.00 \\
\hline & Loss of family** & -2.565932 & 1.131116 & 666.436 & 2.268 & 0.02 \\
\hline & Lack of activities & 0.116151 & 1.236014 & 648.976 & 0.094 & 0.93 \\
\hline & Family issues & 0.931223 & 1.092367 & 656.981 & 0.852 & 0.39 \\
\hline & & & & & - & \\
\hline & Money issues & -0.756502 & 1.277566 & 660.712 & 0.592 & 0.55 \\
\hline & Medical illness & 2.039689 & 1.209101 & 659.205 & 1.687 & 0.09 \\
\hline & Moving/relocation & 0.402597 & 1.269436 & 632.804 & 0.317 & 0.75 \\
\hline & Six month & -2.081391 & 1.311385 & 532.643 & $\begin{array}{r}- \\
1.587\end{array}$ & 0.11 \\
\hline
\end{tabular}




\begin{tabular}{|c|c|c|c|c|c|}
\hline Three-month & -1.917271 & 1.223535 & 519.945 & 1.567 & 0.12 \\
\hline Baseline & . & . & . & & . \\
\hline $\begin{array}{l}\text { Six month * Loss of } \\
\text { family }\end{array}$ & 0.384536 & 1.454112 & 477.706 & 0.264 & 0.79 \\
\hline $\begin{array}{l}\text { Three-month * Loss } \\
\text { of family }\end{array}$ & 2.647652 & 1.375376 & 472.752 & 1.925 & 0.06 \\
\hline $\begin{array}{l}\text { Baseline* Loss of } \\
\text { family }^{\text {a }}\end{array}$ & • & . & . & & . \\
\hline $\begin{array}{l}\text { Six month* Lack of } \\
\text { activities }\end{array}$ & 1.250166 & 1.599032 & 486.057 & 0.782 & 0.44 \\
\hline $\begin{array}{l}\text { Three-month* Lack } \\
\text { of activities }\end{array}$ & 0.795913 & 1.491419 & 460.995 & 0.534 & 0.59 \\
\hline $\begin{array}{l}\text { Baseline* Lack of }^{*} \\
\text { activities }^{b}\end{array}$ & . & . & . & & . \\
\hline $\begin{array}{l}\text { Six month * Family } \\
\text { issues }\end{array}$ & -0.545182 & 1.411993 & 475.245 & $0.38 \overline{6}^{-}$ & 0.70 \\
\hline $\begin{array}{l}\text { Three-month * } \\
\text { Family issues }\end{array}$ & -0.485719 & 1.323691 & 469.325 & 0.367 & 0.71 \\
\hline $\begin{array}{l}\text { Baseline * Family } \\
\text { issues }^{\mathrm{c}}\end{array}$ & & & . & & . \\
\hline
\end{tabular}




\begin{tabular}{|c|c|c|c|c|c|}
\hline $\begin{array}{l}\text { Six month * Money } \\
\text { issues }\end{array}$ & 0.643021 & 1.593569 & 474.516 & 0.404 & 0.69 \\
\hline $\begin{array}{l}\text { Three-month * } \\
\text { Money issues }\end{array}$ & 1.933622 & 1.541237 & 475.628 & 1.255 & 0.21 \\
\hline $\begin{array}{l}\text { Baseline * Money } \\
\text { issues }^{\mathrm{d}}\end{array}$ & • & . & . & & . \\
\hline $\begin{array}{l}\text { Six month* Medical } \\
\text { illness }\end{array}$ & -0.185041 & 1.528062 & 501.802 & 0.121 & 0.90 \\
\hline $\begin{array}{l}\text { Three-month* } \\
\text { Medical illness }\end{array}$ & -0.408363 & 1.470459 & 483.819 & 0.278 & 0.78 \\
\hline $\begin{array}{l}\text { Baseline* Medical } \\
\text { illness }\end{array}$ & . & . & . & . & . \\
\hline $\begin{array}{l}\text { Six month* } \\
\text { Moving/relocation }\end{array}$ & 0.414521 & 1.561209 & 484.155 & 0.266 & 0.79 \\
\hline $\begin{array}{l}\text { Three-month* } \\
\text { Moving/relocation }\end{array}$ & -0.566642 & 1.486248 & 463.815 & 0.381 & 0.70 \\
\hline $\begin{array}{l}\text { Baseline* } \\
\text { Moving/relocation }^{f}\end{array}$ & . & 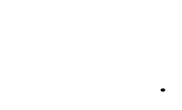 & . & • & . \\
\hline Diabetes & -0.45268 & 0.455217 & 317.745 & 0.994 & 0.32 \\
\hline High blood pressure & -0.61791 & 0.51854 & 339.885 & $1.192^{-}$ & 0.23 \\
\hline
\end{tabular}




\begin{tabular}{|c|c|c|c|c|c|}
\hline Heart trouble & 0.074163 & 0.483269 & 401.182 & 0.153 & 0.88 \\
\hline Suicidal ideation** & 4.444596 & 1.205237 & 721.459 & 3.688 & 0.00 \\
\hline Death ideation $* *$ & 3.252547 & 0.671215 & 711.003 & 4.846 & 0.00 \\
\hline $\begin{array}{l}\text { No suicidal ideation } \\
\text { or death ideation }\end{array}$ & . & & . & . & \\
\hline Anxiety NOS** & 3.346661 & 0.852146 & 702.877 & 3.927 & 0.00 \\
\hline $\begin{array}{l}\text { Generalized anxiety } \\
\text { disorder }\end{array}$ & 0.126621 & 1.71798 & 746.764 & 0.074 & 0.94 \\
\hline Panic disorder & 5.653136 & 2.266859 & 744.583 & 2.494 & 0.01 \\
\hline No anxiety diagnosis & . & & . & . & \\
\hline At-risk drinking & -1.927189 & 1.777936 & 649.264 & 1.084 & 0.28 \\
\hline No at-risk drinking & $\cdot$ & & . & . & \\
\hline Philadelphia, $\mathrm{PA}^{\mathrm{g}}$ & 4.189769 & 1.606126 & 410.346 & 2.609 & 0.01 \\
\hline Chinatown, NY & 6.426349 & 1.182084 & 379.329 & 5.436 & 0.00 \\
\hline Sunset Park, NY** & 11.536024 & 1.382436 & 344.403 & 8.345 & 0.00 \\
\hline Rochester, NY & -0.238426 & 1.740881 & 422.202 & 0.137 & 0.89 \\
\hline San Francisco, CA & . & & . & . & \\
\hline
\end{tabular}




\begin{tabular}{|c|c|c|c|c|c|c|}
\hline & $\begin{array}{l}\text { Enhanced specialty } \\
\text { referral }\end{array}$ & -0.727432 & 0.809162 & 342.594 & 0.899 & 0.37 \\
\hline & Integrated care $^{\mathrm{h}}$ & . & & & . & \\
\hline & & & & & 10.28 & \\
\hline Subthreshold depression & Intercept & 17.413351 & 1.692371 & 383.573 & 9 & 0.00 \\
\hline & Loss of family & 0.491395 & 1.197417 & 406.206 & 0.41 & 0.68 \\
\hline & Lack of activities & -0.298746 & 1.411258 & 406.049 & $0.21 \overline{2}^{-}$ & 0.83 \\
\hline & Family issues & -1.937387 & 1.197079 & 406.646 & $1.618^{-}$ & 0.11 \\
\hline & Money issues & 0.528138 & 1.365233 & 407.941 & 0.387 & 0.70 \\
\hline & Medical illness & -0.923733 & 1.120058 & 404.456 & 0.825 & 0.41 \\
\hline & Moving & -0.949216 & 1.433244 & 404.651 & 0.662 & 0.51 \\
\hline & Six month & -0.379986 & 1.075036 & 271.209 & 0.353 & 0.72 \\
\hline & Three-month & -0.731247 & 1.03984 & 239.068 & 0.703 & 0.48 \\
\hline & Baseline & • & & & . & \\
\hline
\end{tabular}




\begin{tabular}{|c|c|c|c|c|c|}
\hline $\begin{array}{l}\text { Six month * Loss of } \\
\text { family }\end{array}$ & -0.825114 & 1.814056 & 282.715 & $0.455^{-}$ & 0.65 \\
\hline $\begin{array}{l}\text { Three-month * Loss } \\
\text { of family }\end{array}$ & -0.212896 & 1.603124 & 237.35 & $0.133^{-}$ & 0.89 \\
\hline $\begin{array}{l}\text { Baseline* Loss of } \\
\text { family }^{\text {a }}\end{array}$ & & . & . & , & . \\
\hline $\begin{array}{l}\text { Six month* Lack of } \\
\text { activities }\end{array}$ & 0.535328 & 2.002411 & 273.795 & 0.267 & 0.79 \\
\hline $\begin{array}{l}\text { Three-month* Lack } \\
\text { of activities }\end{array}$ & 0.554292 & 1.940905 & 256.292 & 0.286 & 0.78 \\
\hline $\begin{array}{l}\text { Baseline* Lack of } \\
\text { activities }^{b}\end{array}$ & . & . & . & . & \\
\hline $\begin{array}{l}\text { Six month * Family } \\
\text { issues }\end{array}$ & 1.6816 & 1.71381 & 253.772 & 0.981 & 0.33 \\
\hline $\begin{array}{l}\text { Three-month * } \\
\text { Family issues }\end{array}$ & 0.222259 & 1.579417 & 256.247 & 0.141 & 0.89 \\
\hline $\begin{array}{l}\text { Baseline * Family } \\
\text { issues }^{c}\end{array}$ & . & . & . & . & \\
\hline $\begin{array}{l}\text { Six month * Money } \\
\text { issues }\end{array}$ & -0.323352 & 1.855882 & 252.859 & $0.174^{-}$ & 0.86 \\
\hline $\begin{array}{l}\text { Three-month * } \\
\text { Money issues }\end{array}$ & 1.956695 & 1.819324 & 259.205 & 1.076 & 0.28 \\
\hline
\end{tabular}




\begin{tabular}{|c|c|c|c|c|c|}
\hline $\begin{array}{l}\text { Baseline * Money } \\
\text { issues }^{\mathrm{d}}\end{array}$ & & . & . & & . \\
\hline $\begin{array}{l}\text { Six month* Medical } \\
\text { illness }\end{array}$ & 2.161718 & 1.801405 & 292.882 & 1.2 & 0.23 \\
\hline $\begin{array}{l}\text { Three-month* } \\
\text { Medical illness }\end{array}$ & 0.052431 & 1.515124 & 243.808 & 0.035 & 0.97 \\
\hline $\begin{array}{l}\text { Baseline* Medical } \\
\text { illness }\end{array}$ & . & . & . & & . \\
\hline Six month* Moving & 1.974335 & 2.060362 & 310.62 & 0.958 & 0.34 \\
\hline $\begin{array}{l}\text { Three-month* } \\
\text { Moving }\end{array}$ & 1.140545 & 2.000798 & 258.118 & 0.57 & 0.57 \\
\hline Baseline* Moving $^{\mathrm{f}}$ & . & $\cdot$ & . & & . \\
\hline Diabetes & -0.621381 & 0.604949 & 302.716 & 1.027 & 0.31 \\
\hline High blood pressure & -0.294684 & 0.566544 & 287.945 & -0.52 & 0.60 \\
\hline Heart trouble & 0.43149 & 0.453745 & 273.35 & 0.951 & 0.34 \\
\hline Suicidal ideation** & 4.484897 & 1.362886 & 380.841 & 3.291 & 0.00 \\
\hline Death ideation ** & 2.814408 & 0.783364 & 407.599 & 3.593 & 0.00 \\
\hline $\begin{array}{l}\text { No suicidal ideation } \\
\text { or death ideation }\end{array}$ & & & & & \\
\hline
\end{tabular}




\begin{tabular}{|c|c|c|c|c|c|c|}
\hline & Anxiety NOS** & 5.348119 & 1.025245 & 406.437 & 5.216 & 0.00 \\
\hline & $\begin{array}{l}\text { Generalized anxiety } \\
\text { disorder** }\end{array}$ & 5.6059 & 1.570767 & 332.893 & 3.569 & 0.00 \\
\hline & Panic disorder** & 11.768943 & 3.343824 & 405.419 & 3.52 & 0.00 \\
\hline & No anxiety diagnosis & . & . & . & . & . \\
\hline & At-risk drinking & -0.245396 & 1.247951 & 407.994 & 0.197 & 0.84 \\
\hline & No at-risk drinking & & . & . & & . \\
\hline & Philadelphia, $\mathrm{PA}^{\mathrm{g}}$ & 1.751932 & 1.079054 & 251.494 & 1.624 & 0.11 \\
\hline & Chinatown, $\mathrm{NY}^{* *}$ & 2.28003 & 1.079191 & 291.123 & 2.113 & 0.04 \\
\hline & Sunset Park, NY** & 5.385481 & 1.678093 & 252.971 & 3.209 & 0.00 \\
\hline & Rochester, NY & 0.173056 & 1.160172 & 256.362 & 0.149 & 0.88 \\
\hline & San Francisco, CA & . & . & & . & . \\
\hline & $\begin{array}{l}\text { Enhanced specialty } \\
\text { referral }\end{array}$ & -0.841346 & 0.744485 & 270.161 & -1.13 & 0.26 \\
\hline & Integrated care $^{\mathrm{h}}$ & & & & & \\
\hline No depression & Intercept & 12.329281 & 1.494987 & 489.428 & 8.247 & 0.00 \\
\hline & Loss of family** & -5.600933 & 1.649109 & 492.189 & 3.396 & 0.00 \\
\hline
\end{tabular}




$\begin{array}{lrrrrr}\text { Lack of activities } & -2.968602 & 2.006024 & 476.814 & -1.48 & 0.14 \\ \text { Family issues } & 3.457695 & 1.801996 & 473.845 & 1.919 & 0.06 \\ \text { Money issues } & -2.134399 & 2.133584 & 473.686 & -1 & 0.32 \\ \text { Medical illness** } & 5.93548 & 1.545676 & 514.637 & 3.84 & 0.00 \\ \text { Moving } & 3.307664 & 1.857621 & 503.999 & 1.781 & 0.08 \\ \text { Six month } & 5.384308 & 1.142401 & 294.257 & 4.713 & 0.00 \\ \begin{array}{l}\text { Three-month } \\ \text { Baseline }\end{array} & 5.846923 & 1.144707 & 283.004 & 5.108 & 0.00 \\ \begin{array}{l}\text { Six month * Loss of } \\ \text { family** }\end{array} & 6.019229 & 1.644024 & 283.869 & 3.661 & . \\ \begin{array}{l}\text { Three-month * Loss } \\ \text { of family** }\end{array} & 5.573223 & 1.66951 & 268.78 & 3.338 & 0.00 \\ \begin{array}{l}\text { Baseline* Loss of } \\ \text { family }\end{array} & & . & . & . & . \\ \begin{array}{l}\text { Six month* Lack of } \\ \text { activities }\end{array} & 0.387448 & 2.003398 & 283.09 & 0.193 & 0.00 \\ \begin{array}{l}\text { Three-month* Lack } \\ \text { of activities }\end{array} & 2.950558 & 2.028227 & 270.598 & 1.455 & 0.85\end{array}$




\begin{tabular}{|c|c|c|c|c|c|}
\hline $\begin{array}{l}\text { Baseline* Lack of }^{*} \\
\text { activities }^{b}\end{array}$ & & . & . & & \\
\hline $\begin{array}{l}\text { Six month * Family } \\
\text { issues }\end{array}$ & -2.913294 & 1.809472 & 285.056 & -1.61 & 0.11 \\
\hline $\begin{array}{l}\text { Three-month * } \\
\text { Family issues }\end{array}$ & -3.444053 & 1.858994 & 273.191 & 1.853 & 0.07 \\
\hline $\begin{array}{l}\text { Baseline * Family } \\
\text { issues }^{c}\end{array}$ & & . & . & . & . \\
\hline $\begin{array}{l}\text { Six month * Money } \\
\text { issues }\end{array}$ & 4.306441 & 2.134175 & 285.598 & 2.018 & 0.05 \\
\hline $\begin{array}{l}\text { Three-month * } \\
\text { Money issues }\end{array}$ & 2.510761 & 2.181686 & 290.978 & 1.151 & 0.25 \\
\hline $\begin{array}{l}\text { Baseline * Money } \\
\text { issues }^{\mathrm{d}}\end{array}$ & & . & 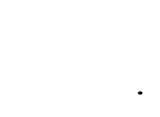 & . & \\
\hline $\begin{array}{l}\text { Six month* Medical } \\
\text { illness** }\end{array}$ & -5.896603 & 1.557143 & 293.704 & 3.787 & 0.00 \\
\hline $\begin{array}{l}\text { Three-month* } \\
\text { Medical illness** }\end{array}$ & -6.030643 & 1.578699 & 278.955 & -3.82 & 0.00 \\
\hline $\begin{array}{l}\text { Baseline* Medical } \\
\text { illness }^{\text {e }}\end{array}$ & & . & . & . & \\
\hline $\begin{array}{l}\text { Six month* } \\
\text { Moving/relocation }\end{array}$ & -2.314841 & 1.871769 & 301.572 & 1.237 & 0.22 \\
\hline
\end{tabular}




\begin{tabular}{|c|c|c|c|c|c|}
\hline $\begin{array}{l}\text { Three-month* } \\
\text { Moving/relocation }\end{array}$ & -1.28455 & 1.905767 & 284.401 & 0.674 & 0.50 \\
\hline $\begin{array}{l}\text { Baseline* } \\
\text { Moving/relocation }^{f}\end{array}$ & & • & • & $\cdot$ & \\
\hline Diabetes & -0.323582 & 0.740002 & 278.512 & 0.437 & 0.66 \\
\hline High blood pressure & 0.719324 & 0.624641 & 270.594 & 1.152 & 0.25 \\
\hline Heart trouble & -0.467801 & 0.566102 & 316.779 & 0.826 & 0.41 \\
\hline Suicidal ideation & 1.206535 & 1.419208 & 398.863 & 0.85 & 0.40 \\
\hline Death ideation & -0.618634 & 0.894913 & 514.868 & $0.691^{-}$ & 0.49 \\
\hline $\begin{array}{l}\text { No suicidal ideation } \\
\text { or death ideation }\end{array}$ & 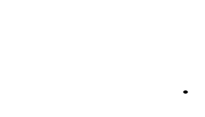 & & . & . & \\
\hline Anxiety NOS** & 3.772582 & 1.14657 & 524.223 & 3.29 & 0.00 \\
\hline $\begin{array}{l}\text { Generalized anxiety } \\
\text { disorder** }\end{array}$ & 4.873769 & 1.56142 & 537.979 & 3.121 & 0.00 \\
\hline Panic disorder & 10.359651 & 5.674335 & 293.973 & 1.826 & 0.07 \\
\hline
\end{tabular}




\begin{tabular}{|c|c|c|c|c|c|}
\hline At-risk drinking & -5.417245 & 0.812415 & 575.341 & 6.668 & 0.00 \\
\hline No at-risk drinking & & & . & & \\
\hline San Francisco, CA & -1.138748 & 1.03965 & 241.731 & 1.095 & 0.27 \\
\hline Chinatown, NY & -4.787927 & 1.156818 & 277.928 & $4.139^{-}$ & 0.00 \\
\hline Sunset Park, NY** & 1.460519 & 1.659392 & 282.833 & 0.88 & 0.38 \\
\hline Rochester, NY & -0.961789 & 1.242552 & 240.998 & 0.774 & 0.44 \\
\hline Philadelphia, $\mathrm{PA}^{\mathrm{g}}$ & . & . & . & $\cdot$ & . \\
\hline $\begin{array}{l}\text { Enhanced specialty } \\
\text { referral }\end{array}$ & -0.553454 & 0.708656 & 260.853 & 0.781 & 0.44 \\
\hline Integrated care ${ }^{\mathrm{h}}$ & & & & & \\
\hline
\end{tabular}

Dependent Variable: Depression severity as measured by the CES-D (Radloff, 1997).

${ }^{a}$ The reference group category is: Baseline* Loss of family.

${ }^{\mathrm{b}}$ The reference group category is: Baseline*Lack of activities.

${ }^{\mathrm{c}}$ The reference group category is: Baseline * Family issues.

${ }^{\mathrm{d}}$ The reference group category is: Baseline * Money issues. 
${ }^{\mathrm{e}}$ The reference group category is: Baseline* Medical illness.

${ }^{\mathrm{f}}$ The reference group category is: Baseline* Moving/relocation.

${ }^{\mathrm{g}}$ The reference group category is: San Francisco, CA.

${ }^{\mathrm{h}}$ The reference group category is: Integrated care. 
Table 4.9

Impact of Gender on Baseline, Three-month, and Six-Month Scores on the CES-D Among Participants Stratified Across Levels of Depression $(N=585)$

\begin{tabular}{|c|c|c|c|c|c|c|}
\hline & Variable & Estimate & Std. Error & $\mathrm{df}$ & $t$ & Sig. \\
\hline \multirow[t]{21}{*}{$\begin{array}{l}\text { Major depressive } \\
\text { disorder }\end{array}$} & Intercept & 27.036438 & 2.189565 & 580.391 & 12.348 & 0.00 \\
\hline & Diabetes & -0.484471 & 0.454432 & 322.1 & -1.066 & 0.29 \\
\hline & High blood pressure & -0.703199 & 0.524054 & 345.554 & -1.342 & 0.18 \\
\hline & Heart trouble & 0.130838 & 0.476442 & 404.535 & 0.275 & 0.78 \\
\hline & Suicidal ideation** & 4.555074 & 1.20602 & 721.748 & 3.777 & 0.00 \\
\hline & Death ideation $* *$ & 3.225832 & 0.663654 & 705.481 & 4.861 & 0.00 \\
\hline & $\begin{array}{l}\text { No suicidal ideation or death } \\
\text { ideation }\end{array}$ & & & & & \\
\hline & Anxiety NOS** & 3.366175 & 0.841906 & 697.894 & 3.998 & 0.00 \\
\hline & Generalized anxiety disorder & -0.103024 & 1.713711 & 746.572 & -0.06 & 0.95 \\
\hline & Panic disorder** & 4.962121 & 2.254873 & 745.182 & 2.201 & 0.03 \\
\hline & No anxiety diagnosis & & & & & \\
\hline & At-risk drinking & -1.984083 & 1.784911 & 653.861 & -1.112 & 0.27 \\
\hline & No at-risk drinking & & & & & \\
\hline & Female & -1.146602 & 1.09976 & 663.059 & -1.043 & 0.30 \\
\hline & Male & & & & & \\
\hline & Philadelphia, $\mathrm{PA}^{\mathrm{c}}$ & 3.536086 & 1.546103 & 422.249 & 2.287 & 0.02 \\
\hline & Chinatown, $\mathrm{NY}^{* *}$ & 6.787645 & 1.095768 & 382.962 & 6.194 & 0.00 \\
\hline & Sunset Park, NY ** & 11.970337 & 1.331117 & 356.05 & 8.993 & 0.00 \\
\hline & Rochester, NY & 0.203261 & 1.706544 & 428.84 & 0.119 & 0.91 \\
\hline & San Francisco, CA & & & & & \\
\hline & six-month** & -4.362871 & 1.201222 & 494.125 & -3.632 & 0.00 \\
\hline
\end{tabular}




\begin{tabular}{|c|c|c|c|c|c|c|}
\hline & $\begin{array}{l}\text { three-month } \\
\text { Baseline }^{\mathrm{d}}\end{array}$ & -1.779963 & 1.14888 & 492.612 & -1.549 & 0.12 \\
\hline & Six month* Female** & 3.988958 & 1.438535 & 489.71 & 2.773 & 0.01 \\
\hline & Six month* Male ${ }^{\mathrm{e}}$ & & & & & \\
\hline & Three-month*Female & 1.52359 & 1.363413 & 485.833 & 1.117 & 0.26 \\
\hline & Three-month* Male $^{\mathrm{f}}$ & . & 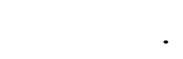 & . & . & \\
\hline & Baseline* Female ${ }^{\mathrm{g}}$ & . & . & 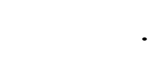 & • & \\
\hline & Baseline*Male & & & & & \\
\hline & Enhanced speciality refferal & -0.726326 & 0.799027 & 350.894 & -0.909 & 0.36 \\
\hline & Integrated care $^{\mathrm{h}}$ & & & & 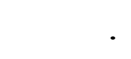 & \\
\hline $\begin{array}{l}\text { Subthreshold } \\
\text { depression }\end{array}$ & Intercept & 16.369408 & 1.68377 & 386.5 & 9.722 & 0.00 \\
\hline & Diabetes & -0.542757 & 0.598546 & 302.616 & -0.907 & 0.37 \\
\hline & High blood pressure & -0.403937 & 0.564685 & 291.08 & -0.715 & 0.48 \\
\hline & Heart trouble & 0.524213 & 0.448169 & 271.964 & 1.17 & 0.24 \\
\hline & Suicidal ideation** & 4.479217 & 1.37716 & 389.924 & 3.253 & 0.00 \\
\hline & Death ideation $* *$ & 2.788448 & 0.784272 & 405.449 & 3.555 & 0.00 \\
\hline & $\begin{array}{l}\text { No suicidal ideation or death } \\
\text { ideation }\end{array}$ & & & & & \\
\hline & Anxiety NOS** & 5.199287 & 1.013475 & 407.049 & 5.13 & 0.00 \\
\hline & Generalized anxiety & & & & & \\
\hline & disorder** & 5.772777 & 1.56508 & 334.373 & 3.688 & 0.00 \\
\hline & Panic disorder** & 11.885374 & 3.302694 & 405.939 & 3.599 & 0.00 \\
\hline & No anxiety diagnosis & & & & & \\
\hline & At-risk drinking & -0.452088 & 1.255788 & 407.861 & -0.36 & 0.72 \\
\hline & No at-risk drinking & & & & & \\
\hline & Female & 0.284637 & 1.041853 & 405.849 & 0.273 & 0.79 \\
\hline & Male & & & & & \\
\hline & Philadelphia, $\mathrm{PA}^{\mathrm{c}}$ & 1.914461 & 1.018568 & 252.89 & 1.88 & 0.06 \\
\hline & Chinatown, NY & 2.185559 & 1.045444 & 297.527 & 2.091 & 0.04 \\
\hline
\end{tabular}




\begin{tabular}{|c|c|c|c|c|c|c|}
\hline & Sunset Park, NY & 4.737176 & 1.596106 & 261.865 & 2.968 & 0.00 \\
\hline & Rochester, NY & -0.169535 & 1.059983 & 264.234 & -0.16 & 0.87 \\
\hline & San Francisco, CA & & & & & \\
\hline & six-month & 0.554927 & 1.277505 & 260.371 & 0.434 & $\begin{array}{l}0.66 \\
0.88\end{array}$ \\
\hline & $\begin{array}{l}\text { three-month } \\
\text { Baseline }^{\mathrm{d}}\end{array}$ & 0.182055 & 1.198368 & 258.374 & 0.152 & 0.88 \\
\hline & $\begin{array}{l}\text { Six month* Female } \\
\text { Six month* Male }\end{array}$ & 1.163445 & 1.542505 & 273.535 & 0.754 & 0.45 \\
\hline & Three-month*Female & 0.342517 & 1.457183 & 257.323 & 0.235 & 0.81 \\
\hline & Three-month* Male ${ }^{f}$ & . & 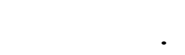 & 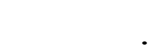 & . & \\
\hline & Baseline* Female $\mathrm{g}^{\mathrm{g}}$ & 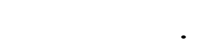 & 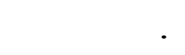 & & & \\
\hline & Baseline*Male & & & & & \\
\hline & $\begin{array}{l}\text { Enhanced specialty referral } \\
\text { Integrated care }\end{array}$ & -0.727432 & 0.809162 & 342.594 & -0.899 & 0.37 \\
\hline No depression & Intercept & 13.708019 & 1.357818 & 425.682 & 10.096 & 0.00 \\
\hline & Diabetes & -0.20972 & 0.743649 & 267.264 & -0.282 & 0.78 \\
\hline & High blood pressure & 0.47978 & 0.623802 & 256.387 & 0.769 & 0.44 \\
\hline & Heart trouble & -0.352703 & 0.579132 & 307.232 & -0.609 & 0.54 \\
\hline & Suicidal ideation & 0.608231 & 1.48258 & 417.847 & 0.41 & 0.68 \\
\hline & Death ideation & -1.079292 & 0.910286 & 529.752 & -1.186 & 0.24 \\
\hline & $\begin{array}{l}\text { No suicidal ideation or death } \\
\text { ideation }\end{array}$ & & & & & \\
\hline & Anxiety NOS** & 4.001433 & 1.195219 & 548.082 & 3.348 & 0.00 \\
\hline & $\begin{array}{l}\text { Generalized anxiety } \\
\text { disorder** }\end{array}$ & 5.613431 & 1.618598 & 548.468 & 3.468 & 0.00 \\
\hline & Panic disorder** & 10.314953 & 5.935178 & 292.204 & 1.738 & 0.08 \\
\hline & No anxiety diagnosis & & & & & \\
\hline & At-risk drinking** & -5.241497 & 0.835023 & 574.719 & -6.277 & 0.00 \\
\hline & No at-risk drinking & & & & & \\
\hline & Female & -0.376266 & 1.414625 & 511.673 & -0.266 & 0.79 \\
\hline
\end{tabular}




\section{Male}

Philadelphia, $\mathrm{PA}^{\mathrm{c}}$

Chinatown, $\mathrm{NY}^{* *}$

0.821715

$-3.297386$

0.992835

1.010481

224.413

0.828

0.41

Sunset Park, NY

3.110083

1.570275

283.474

$-3.263$

0.00

Rochester, NY

San Francisco, CA

1.055412

1.021872

1.981

0.05

six-month**

three-month**

Baseline $^{\mathrm{d}}$

4.69898

0.30

1.033

0.00

$\begin{array}{lll}4.873173 & 1.007791 & 241.962\end{array}$

4.593

0.00

Six month* Female

$-0.595693$

1.411882

277.515

4.836

0.67

Six month* Male ${ }^{\mathrm{e}}$

Three-month*Female

$-0.12654$

1.443862

272.129

$-0.422$

Three-month* Male $^{\mathrm{f}}$

Baseline* Female ${ }^{\mathrm{g}}$

Baseline*Male

Enhanced specialty referral

$-0.321425$

0.708629

244.639

$-0.088$

0.93

Integrated care

${ }^{a}$ The reference group category is: No depression.

${ }^{\mathrm{b}}$ The reference group

category is: Female.

${ }^{\mathrm{c}}$ The reference group category is: San Francisco, CA.

$\mathrm{d}$ The reference group

category is: Baseline.

${ }^{\mathrm{e}}$ The reference group category is: Six month* Male.

${ }^{\mathrm{f}}$ The reference group category is: Three-month* Male.

${ }^{\mathrm{g}}$ The reference group category is: Baseline* Male

$* * p<.05$

Table 4.10 
Impact of the Interaction Between Gender and Frequency of Life Stressors on Baseline, Three-Month, and Six-Month Scores on the CES-D Among Participants Stratified Across Levels of Depression ( $N=585)$

\begin{tabular}{|c|c|c|c|c|c|c|c|c|c|}
\hline Diagnosis & Variable & Estimate & SE & df & $t$ & Sig. & Baseline & $\begin{array}{l}\text { three- } \\
\text { month }\end{array}$ & six-month \\
\hline \multirow[t]{4}{*}{ MDD } & Family issues & 4.511504 & 2.331678 & 659.76 & 1.935 & 0.053 & $\mathrm{X}$ & & \\
\hline & Money issues & 6.151675 & 3.070954 & 734.298 & 2.003 & 0.046 & & \multirow{3}{*}{$\mathrm{X}$} & $\mathrm{X}$ \\
\hline & Medical illness & 5.754936 & 2.812123 & 728.325 & 2.046 & 0.041 & & & \\
\hline & Lack of activities/work & 6.241737 & 3.096431 & 734.247 & -2.016 & 0.044 & & & $\mathrm{X}$ \\
\hline \multirow[t]{2}{*}{ SD } & $\begin{array}{l}\text { Loss of family and } \\
\text { friends }\end{array}$ & 9.443049 & 3.682305 & 384.862 & 2.564 & 0.011 & & $\mathrm{X}$ & \\
\hline & Lack of activities/work & 8.262339 & 3.803465 & 402.07 & -2.172 & 0.03 & & $\mathrm{X}$ & \\
\hline
\end{tabular}

Dependent Variable: Depression severity as measured by the CES-D (Radloff, 1997). 


\section{References}

Adams, K., Leibbrandt, S., \& Moon, H. (2011). A critical review of the literature on social and leisure activity and wellbeing in later life. Ageing and Society, 31, 683712.

Administration on Aging (2004). National Family Caregiver Support Program (FCSP) Complete Resource Guide. Washington, DC: Author.

Afshartous, D. (1995, April). Determination of sample size for multilevel model design. Paper presented at the annual meeting of the American Educational Research Association, San Francisco, CA.

Agahi, N. \& Parker, M. (2008). Leisure activities and mortality: Does gender matter? Journal of Aging and Health, 20(7), 855-871.

Akaike, H. (1974). A new look at the statistical model identification. IEEE Transactions on Automatic Control, 19(6), 716-723. doi:10.1109/TAC.1974.1100705

American Psychiatric Association. (2000). Diagnostic and statistical manual of mental disorders. 4th ed. Washington, DC: American Psychiatric Association.

American Psychiatric Association. (2013). Diagnostic and statistical manual of mental disorders. 5th ed. Washington, DC: American Psychiatric Association.

Anderson, R., Freedland, K., Clouse, R., \& Lustman, P. (2001). The prevalence of comorbid depression in adults with diabetes: a meta-analysis. Diabetes Care, 24(6), 1069-78.

Andrew, M., Mitnitski, A., Kirkland, S., \& Rockwood, K. (2012). The impact of social vulnerability on the survival of the fittest older adults. Age and Ageing. doi: 10.1093/ageing/afr176

Atchley, R. (1989). A continuity theory of normal aging. The Gerontologist, 29(2), 183190.

Avison, W. (1996). Social networks as risk and protective factors for onset and recurrence of mental disorders. Current Opinion in Psychiatry, 9(2), 149-152.

Baltes, P. (1987). Theoretical propositions of life span developmental psychology: On the dynamics between growth and decline. Developmental Psychology, 23(5), 611626.

Baltes, P., Lindenberger, U., \& Staudinger, U. M. (2006). Lifespan theory in developmental psychology. In R. M. Lerner (Ed.). Handbook of child psychology (pp. 569-664). New York, NY: John Wiley \& Sons, Inc. 
Bartels, S., Coakley, E., Zubritsky, C., Ware, J., Miles, K., Areán, P., Chen, H., Oslin, D., Llorente, M., Costantino, G., Quijano, L., McIntyre, J., Linkins, K., Oxman, T., Maxwell, J., \& Levkoff, S. (2004). Improving access to geriatric mental health services: a randomized trial comparing treatment engagement with integrated versus enhanced referral care for depression, anxiety, and at-risk alcohol use. American Journal of Psychiatry, 161 (8), 1455-62.

Beck, A. T., Epstein, N., Brown, G., \& Steer, R. A. (1988). An inventory for measuring clinical anxiety: Psychometric properties. Journal of Consulting and Clinical Psychology, 56(6), 893-897.

Becker, E., Margraf, J., Turke, V., Soeder, U., \& Neumer, S. (2001). Obesity and mental illness in a representative sample of young women. International Journal of Obesity and Related Metabolic Disorders, 25 (Suppl. 1), S5-9.

Bickel, R. (2007). Multilevel analysis for applied research: It's just regression! New York: The Guilford Press.

Bierman, A., Fazio, E.M., \& Milkie, M.A. (2006). A multifaceted approach to the mental health advantage of the married: Assessing how explanations vary by outcome measure and unmarried group. Journal of Family Issues, 27(4), 554-582.

Bijou, S. (1993). Behavior analysis of child development (2nd ed.). Reno, NV: Context Press.

Blazer, D. (2003). Depression in late life: Review and commentary. The Journals of Gerontology Series A: Biological Sciences and Medical Sciences, 58(3), 249-265.

Blazer, D. (2009). Depression in late life: Review and commentary. The Journal of Life Long Learning in Psychiatry. 7, 118-136.

Blazer, D.G. \& Steffens, D.C. (Eds). (2009). Textbook of geriatric psychiatry. Arlington, VA: American Psychiatric Publishing Inc.

Blow, F. C., Gillespie, B.W., Barry, K. L., Mudd, S. A.,\& Hill, E. M. (1998). Brief screening for alcohol problems in elderly populations using the Short Michigan Alcohol Screening Test- Geriatric Version (SMAST-G). Alcoholism: Clinical and Experimental Research, 22 (Suppl.131A).

Boswell, E. \& Stoudemire, A. (1996). Major depression in the primary care setting. American Journal of Medicine, 101(6A), 3S-9S.

Brodaty, H., Luscombe, G., Parker, G., Wilhelm, K., Hickie, I., Austin, M., \& Mitchell, P. (2001). Early and late onset depression in old age: Different aetiologies, same phenomenology. Journal of Affective Disorders, 66(2), 225-236. 
Bruce, M. (2001). Depression and disability in late life: Directions for future research. American Journal of Geriatric Psychiatry, 9(2), 102-112.

Bruce, M. (2002). Psychosocial risk factors for depressive disorders in late life. Biological Psychiatry, 52(3), 175-184.

Bryk, A.S. \& Raudenbush, S.W. (1992). Hierarchical linear models: Applications and data analysis methods. Sage: Newbury Park, CA.

Burnam, M. \& Watkins, K. (2006). Substance abuse with mental disorders: Specialized public systems and integrated care. Health Affairs, 25(3), 648-658. doi:10.1377/hlthaff.25.3.648

Burt, S. \& Stein, K. (2002). Epidemiology of depression throughout the female life cycle. The Journal of Clinical Psychiatry, 63 (7), 9-15.

Bush, P., Zuckerman, A., Theiss, P., Taggart, V., Horowitz, C., Sheridan, M., \& Walter, H. (1989). Cardiovascular risk factor prevention in black school children: Twoyear results of the "know your body" program. American Journal of Epidemiology, 129(3), 466-482.

Butters, M., Whyte, E., Nebes, R., Begley, A., Dew, M., Mulsant, B.H., Zmuda, M.D., Bhalla, R., Meltzer, C.C., Pollock, B.G., Reynolds, C.F., \& Becker, J.T. (2004). The nature and determinants of neuropsychological functioning in late-life depression. Archives of General Psychiatry, 61(6), 587-95.

Carney, R. \& Freedland, K. (2003). Depression, mortality, and medical morbidity in patients with coronary heart disease. Biological Psychiatry, 54(3), 241-247.

Centers for Disease Control and Prevention, (2005). National Center for Injury Prevention and Control. Web-based Injury Statistics Query and Reporting System (WISQARS) [online]. Available at: www.cdc.gov/ncipc/wisqars.

Centers for Disease Control and Prevention, (2010). National Center for Injury Prevention and Control. Web-based Injury Statistics Query and Reporting System (WISQARS) [online]. Available at www.cdc.gov/injury/wisqars/index.html.

Centers for Medicare and Medicaid Services, "Medicare Enrollment-All Beneficiaries: As of July 2003," 17 September 2004,www.cms.hhs.gov/statistics/enrollment/st03all.asp (15 June 2005).

Cervantes, V., Santana, A., Guilera, G., \& Gómez-Benito, J. (2009). Hierarchical linear models in psychiatry: A bibliometric study. Scientometrics, 80(3), 797-808.

Christensen, H., Jorm, A., Mackinnon, A., Korten, A., Jacomb, P., Henderson, A. \& Rodgers, B. (1999). Age differences in depression and anxiety symptoms: A 
structural equation modelling analysis of data from a general population sample. Psychological Medicine, 29, 325-39.

Cole, M. \& Dendukuri, N. (2003). Risk factors for depression among elderly community subjects: A systematic review and meta-analysis. American Journal of Psychiatry, 160(6), 1147-1156.

Cuijpers, P., van Straten, A., \& Warmerdam, L. (2007). Behavioral activation treatments of depression: A meta-analysis. Clinical Psychology Review, 27(3), 318-326.

Dawson, D. A. (2000). U.S. low-risk drinking guidelines: An examination of four alternatives. Alcoholism: Clinical and Experimental Research, 24, 12, 1820-1829.

De Beurs, E., Beekman, A. \& Geerlings, R. (2001). On becoming depressed or anxious in late life: Similar vulnerability factors but different effects of stressful life stress. British Journal of Psychiatry, 179, 426-431. doi: 10.1192/bjp.179.5.426

Djernes, J. (2006). Prevalence and predictors of depression in populations of elderly: A review. Acta Psychiatrica Scandinavica,113(5), 372-387.

Drake, R.E., Mercer-McFadden, C., Mueser, K. T., McHugo, G.J., \& Bond, G.R. (1998). Review of integrated mental health and substance abuse treatment for patients with dual conditions. Schizophrenia Bulletin, 24, 589-608.

Dupree, L., Broskowski, H., \& Schonfeld, L. (1984). The Gerontology Alcohol Project: A behavioral treatment program for elderly alcohol abusers. Gerontologist, 24, 510-516.

Eden, J., Maslow, K., Le, M., \& Blazer, D. (2012). The Mental Health and Substance Use Workforce for Older Adults: In Whose Hands? Washington, DC.: Institute of Medicine.

Egede, L., Zheng, D., \& Simpson, K. (2002). Comorbid depression is associated with increased healthcare use and expenditures in individuals with diabetes. Diabetes Care, 25(3), 464-70.

Engels, J. \& Diehr, P. (2003). Imputation of missing longitudinal data: A comparison of methods. Journal of Clinical Epidemiology, 56 (10), 968-76.

Fairclough, D., Peterson, H., \& Chang, V. (1998). Why are missing quality of life data a problem in clinical trials of cancer therapy? Statistics in Medicine, 17 (5), 667677.

Feder, J., Komisar, H., \& Niefeld, M. (2000). Long-term care in the United States: An overview. Health Affairs, 19 (3), 40-56. 
Field, A. (2013). Discovering statistics using IBM SPSS statistics. London: Sage Publications.

Fiske, A., Gatz, M., \& Pedersen, N. (2003). Depressive symptoms and aging: The effects of illness and non-health related events. The Journals of Gerontology Series B: Psychological Sciences and Social Sciences, 58 (6), P3208.

Fiske, A., Wetherell, J., \& Gatz, M. (2009). Depression in older adults. Annual Review of Clinical Psychology, 5, 363-389. doi: 10.1146/annurev.clinpsy.032408.153621

Gallo, J., Anthony, J., \& Muthén, B. (1994). Age differences in the symptoms of depression: A latent trait analysis. Journal of Gerontology, 49(6), P251-64.

Gallo, J. J. \& Rabins, P. V. (1999). Depression without sadness: Alternative presentations of depression in late life. American Family Physician, 60(3), 820-826.

Gallo, J., Zubritsky, C., Maxwell, J., Nazar, M., Bogner, H., Quijano, L., Syropoulos, H., Cheal, K., Chen, H., Sanchez, H., Dodson, J., \& Levkoff, S. (2004). Primary care clinicians evaluate integrated and referral models of behavioral healthcarefor older adults: Results from a multisite effectiveness trial (PRISM-E). Annals of Family Medicine, 2(4), 305-309.

Garmezy, N. (1991). Resiliency and vulnerability to adverse developmental outcomes associated with poverty. American Behavioral Scientist, 34 (4), 416430. doi: 10.1177/0002764291034004003.

Garson, G. (2013). Hierarchical linear modeling: Guide and applications. Los Angeles: Sage.

Gitterman, A. (2011). Advances in the life model of social work practice. In F. J. Turner (Ed.), Social work treatment: Interlocking theoretical perspectives (5th ed.) (pp. 279-292). New York, NY: Oxford University Press.

Glass, T., Kasl, S., \& Berkman, L. (1997). Stressful life stress and depressive symptoms among the elderly. Evidence from a prospective community study. Journal of Aging and Health, 9(1), 70-89.

Glass, T. A., Mendes de Leon, C. F., Bassuk, S. S. \& Berkman, L. F. (2006). Social engagement and depressive symptoms in late life: Longitudinal findings. Journal of Aging and Health, 18(4), 604-28.

Glass, T. A., Prigerson, H., Kasl, S. V., \& Mendes de Leon, C. F. (1995). The effects of negative life stress on alcohol consumption among older men and women. Journal of Gerontology, 40, 565-571. 
Godin, O., Elbejjani, M., \& Kaufman, J. (2012). Body mass index, blood pressure, and risk of depression in the elderly: A marginal structural model. American Journal of Epidemiology. doi: 10.1093/aje/kws003

Goldberg, D. P., Oldehinkel, T., \& Ormel, J. (1998). Why GHQ threshold varies from one place to another. Psychological Medicine, 28, 915-921.

Goldberg, D. P. \& Williams, P. (1988). A user's guide to the General Health Questionnaire. Windsor, UK: NFER-Nelson Publishing.

Hagerty, B. M. \& Patusky, K. L. (2003). Reconceptualizing the Nurse-Patient relationship. Journal of Nursing Scholarship, 35, 145-150.

Han, B., Gfroerer, J., Colliver, J., \& Penne, M. (2009). Substance use disorder among older adults in the United States in 2020. Addiction, 104(1), 88-96. doi: $10.1111 / \mathrm{j} .1360-0443.2008 .02411$.

Harlow, R. E. \& Cantor, N. (1996). Still participating after all these years: a study of life task participation in later life. Journal of Personality and Social Psychology, 71(6), 1235-49.

Havassy, B.E., Shopshire, M.S., \& Quigley, L.A. (2000). Effects of substance dependence on outcomes of patients in randomized trial of two case management models. Psychiatric Services, 51, 639-644.

Hawkins, J. D., Catalano, R. F., \& Miller J. Y. (1992). Risk and protective factors for alcohol and other drug problems in adolescence and early adulthood: Implications for substance abuse prevention. Psychology Bulletin, 112(1), 64-105.

Hays, J., Saunders, W., Flint, E., Kaplan, B., \& Blazer, D. (1997). Depression and social support as risk factors for functional disability in late life. Aging and Mental Health, 3, 209-220.

Heikkinen, R. \& Kauppinen, M. (2004). Depressive symptoms in late life: A 10-year follow-up. Archives of Gerontology and Geriatrics, 38(3), 239-250.

Hess, D. (2009). Access to public transit and its influence on ridership for older adults in two U.S. cities. Journal of Transport and Land Use, 2(1), 3-27.

Holmes, T. \& Rahe, R. (1967). Holmes-Rahe social readjustment rating scale. Journal of Psychosomatic Research, 11, 213-218. doi: 10.1016/0022-3999(67)90010-4 
Hong, S., Hasche, L., \& Bowland, S. (2009). Structural relationships between social activities and longitudinal trajectories of depression among older adults. The Gerontologist, 49(1), 1-11. doi: 10.1093/geront/gnp006.

Hox, J. (1995). Applied multilevel analysis. TT Publicaties, Amsterdam.

Hoyert, D. \& Xu, J. (2012). Deaths: Preliminary data for 2011. National Vital Statistics Reports, 61, 6.

Hughes, M. \& Waite, L. (2009). Marital biography and health at mid-life. Journal of Health and Social Behavior, 50(3), 344-358. doi: 10.1177/002214650905000307

Hunsley, J. \& Mash, E. (2008). A guide to assessments that work. New York: Oxford University Press.

IBM SPSS Statistics (SPSS) [computer program]. (2010). Version 19. United States: IBM.

Institute of Medicine. (1994). Reducing Risks for Mental Disorders: Frontiers for Preventive Intervention Research. In: Mrazek PJ, Haggerty RJ, editors. Committee on Prevention of Mental Disorders, Division of Biobehavorial Sciences and Mental Disorders. Washington, DC: National Academy Press.f

Janke, M. \& Davey, A. (2006). Implications of selective optimization with compensation on the physical, formal and informal leisure patterns of adults. Indian Journal of Gerontology, 20(1/2), 51-66.

Janke, M., Davey, A. \& Kleiber, D. (2006). Modeling change in older adults' leisure activities. Leisure Studies, 28(3), 285-303.

Jones, A. (2002). The national nursing home survey: 1999 summary. Vital Health Statistics, 13(152), 1-116.

Jones, M. G., Taylor, A. R., \& Broadwell, B. (2009). Concepts of scale held by students with visual impairment. Journal of Research in Science Teaching, 46(5), 506519. doi: $10.1002 /$ tea. 20277

Johnson, R.W. \& Wiener, J. (2006). A profile of frail older Americans and their caregivers. The Retirement Project Occasional Paper, 8. Washington, DC: The Urban Institute. Available at: http://www.urban.org/url.cfm?ID=311284.

Katon, W. \& Kroenke, K. (2007). The association of depression and anxiety with medical symptom burden in patients with chronic medical illness. General Hospital Psychiatry, 29(2), 147-155. 
Katon,W.,VonKorff, M., Lin, E.,Walker, E., Simon, G. E., Bush, T., Robinson, P., \& Russo, J. (1995). Collaborative management to achieve treatment guidelines. Impact on depression in primary care. Journal of the American Medical Association, 273, 1026-1031.

Katzman, R., Brown, T., Fuld, P., Peck, A., Schechter, R., \& Schimmell, H. (1983). Validation of a short Orientation-Memory Concentration Test of cognitive impairment. American Journal of Psychiatry, 140, 734-739.

Kessler, R. (1997). The effects of stressful life stress on depression. Annual Review of Psychology, 48(1), 191-214. doi: 10.1146/annurev.psych.48.1.191

Kessler, R., Berglund, P., Demler, O., Jin, R., Koretz, D., Merikangas, K., Rush, A., Walters, E., \& Wang, P. (2003). The epidemiology of major depressive disorder: results from the National Comorbidity Survey Replication (NCS-R). Journal of the American Medical Association, 289(23), 3095-105.

Kessler, R., McGonagle, K., Nelson, C.B., Hughes, M., Swartz, M., \& Blazer, D. (1994b). Sex and depression in the National Comorbidity Survey II: Cohort effects. Journal of Affective Disorders. 30, 15-26.

Kessler, R.C., McGonagle, K.A., Swartz, M., Blazer, D.G., \& Nelson, C.B. (1993). Sex and depression in the National Comorbidity Survey I: Lifetime prevalence, chronicity and recurrence. Journal of Affective Disorders, 29, 85-96.

Kessler, R.C., McGonagle, K.A., Zhao, S., Nelson, C.B., Hughes, M., Eshleman, S., Wittchen, H.U., \& Kendler, K.S. (1994a). Lifetime and 12-months prevalence of DSM-III R psychiatric disorders in the United States: Results from the National Comorbidity Survey. Archives of General Psychiatry, 51, 8-19.

Keyes, C. (2005). Mental illness and/or mental health? Investigating axioms of the complete state model of health. Journal of Consulting and Clinical Psychology, 73(3), 539-548. doi: 10.1037/0022-006X.73.3.539

Kim, T., Solomon, P., \& Zurlo, K. (2009). Applying Hierarchical Linear Modeling (HLM) to Social Work Administration Research. Administration in Social Work, 33, 3. doi:10.1080/03643100902987739

Krahn, D., Bartels, S., Coakley, E., Oslin, D., Chen, H., McIntyre, J., Chung, H., Maxwell, J., Ware, J., \& Lewkoff, S. (2006). PRISM-E: Comparison of integrated care and enhanced specialty referral models in depression outcomes. Psychiatric Services, 57(7), 946-953. 
Knight, R. G., Williams, S., McGee, R., \& Olaman, S. (1997). Psychometric properties of the Center for Epidemiologic Studies Depression Scale (CES-D) in a sample of women in middle life. Behavior Research \& Therapy, 35(4), 373-380.

Kockler, M., \& Heun, R. (2002). Gender differences of depressive symptoms in depressed and nondepressed elderly persons. International Journal of Geriatric Psychiatry, 17(1), 65-72.

Komaroff, A., Masuda, M., \& Holmes, T. (1968). The social readjustment rating scale: A comparative study of Negro, Mexican, and White Americans. Journal of Psychosomatic Research, 12(2), 121-8. doi: 10.1016/0022-3999(68)90018-4

Kraaij, V., Arensman, E., \& Spinhoven, P. (2002). Negative life stress and depression in elderly persons: A meta-analysis. The Journals of Gerontology Series B:

Psychological Sciences and Social Sciences, 57 (1), 87-P94. doi: 10.1093/geronb/57.1.P87

Kraaij, V. \& de Wilde, E. (2001). Negative life stress and depressive symptoms in the elderly: A life span perspective. Aging \& Mental Health, 5(1), 84-91.

Kraemer, H.C., Kazdin, A.E., Offord, D.R., Kessler, R.C., Jensen, P.S., \& Kupfer, D.J. (1997). Coming to terms with the terms of risk. Archives of General Psychiatry, 54(4), 337-43.

Kreft, I. G. G., \& De Leeuw, J. (1998). Introducing multilevel modeling. Newbury Park, CA: Sage.

Lacro, J. \& Jeste, D. (1994). Physical comorbidity and polypharmacy in older psychiatric patients. Biological Psychiatry, 36, 146-152. doi.org/10.1016/00063223(94)91220-3

Lampinen, P., Heikkinen, R.-L., Kauppinen, M., \& Heikkinen, E. (2006). Activity as a predictor of mental well-being among older adults. Aging and Mental Health, 10(5), 454-66.

Lavretsky, H. (1998). Late-life depression: Risk factors, treatment, and sex differences. Clinical Geriatrics, 6(3), 13-30.

Lecrubier, Y., Sheehan, D. , Weiller, E. , Amorim, P. , Bonora, I., Harnett Sheehan, K., Janavs, J., \& Dunbar, G. (1997). The mini international neuropsychiatric interview (MINI). A short diagnostic structured interview: Reliability and validity according to the CIDI. European Psychiatry, 12(5), 224-231.

Lee, C. \& Gramotnev, H. (2007). Life transitions and mental health in a national cohort of young Australian women. Developmental Psychology, 43(4), 877-888. 
Lemon, B. W., Bengtson, V. L. \& Peterson, J. A. 1972. An exploration of the activity theory of aging: Activity types and life satisfaction among in-movers to a retirement community. Journal of Gerontology, 27(4), 511-23.

Lennon, T. (2004). Statistics on Social Work Education in the United States: 2002. Alexandria, VA: Council on Social Work Education.

Levkoff, S., Chen, H., Coakley, E., McDonel Herr, E., Oslin, D., Katz, I., Bartels, S., Maxwell, J., Olsen, E., Miles, K., Costantino, G., \& Ware, J. (2004). Design and sample characteristics of the PRISM-E multisite randmized trial to improve behavioral healthcarefor the elderly. Journal of Aging and Health, 16(1), 3-27. doi: $10.1177 / 0898264303260390$

Lewinsohn, P.M., Seeley, J.R., Roberts, R.E., \& Allen, N.B. (1997). Center for Epidemiological Studies-Depression Scale (CES-D) as a screening instrument for depression among community-residing older adults. Psychology and Aging, 12(2), 277- 287.

Li, C., Ford, E., Strine, T., \& Mokdad, A. (2008). Prevalence of depression among U.S. adults with diabetes: findings from the 2006 behavioral risk factor surveillance system. Diabetes Care, 31 (1), 105-107.

Li, Y. \& Ferraro, K.F. (2005). Volunteering and depression in later life: Social benefit or selection processes? Journal of Health and Social Behavior, 46(1), 68-84.

Livingston, G., Manela, M., \& Katona, C. (1996). Depression and other psychiatric morbidity in careers of elderly people living at home. British Medical Journal, 312(7024), 153-156.

Lutzky, S. \& Knight, B. (1994). Explaining gender differences in caregiver distress: The roles of attentiveness to emotions and coping styles. Psychology and Aging, 9(4), $513-519$.

Magaziner, J., Simonsick, E., \& Kashner, M. (1990). Predictors of functional recovery in the years following hospital discharge for hip fracture. Journal of Gerontology: Medical Sciences, 45(3), M101-M107.

Maas, C. \& Hox, J. (2005). Sufficient sample sizes for multilevel modeling. Methodology, 1 (3), 86-92. doi:10.1027/1614-1881.1.3.86

Miller, M., Paradis, C., Houck, P. Rifai, A., Mazumdar, S., Pollock, B., Perel, J., Frank, E., \& Reynolds, C. (1996). Chronic medical illness in patients with recurrent major depression. American Journal of Geriatric Psychiatry, 4(4), 281-290. http://dx.doi.org/10.1097/00019442-199622440-00002 
Mojtabai, R. \& Olfson, M. (2004). Major depression in community-dwelling middle-aged and older adults: Prevalence and 2-year and 4-year follow-up symptoms.

Psychological Medicine, 34(4), 623-34.

Mueser, K.T., Noordsy, D., Drake, R., \& Fox, L. (2003). Integrated treatment for dual conditions: A guide to effective practice. New York: Guilford Press.

Nolen-Hoeksema, S., \& Ahrens, C. (2002). Age differences and similarities in the correlates of depressive symptoms. Psychology and Aging, 17, 116-124.

Nolen-Hoeksema, S., Larson, J., \& Grayson, C. (1999). Explaining the gender difference in depressive symptoms. Journal of Personality and Social Psychology, 77(5), 1061-1072.

O'Connell, Boat, \& Warner, K. (2009). Preventing mental, emotional, and behavioral disorders among young people: Progress and possibilities. Washington (DC): National Academies Press (US); 2009. 4, Using a Developmental Framework to Guide Prevention and Promotion. Available from:

http://www.ncbi.nlm.nih.gov/books/NBK32792/

O'Connor, E., Whitlock, E., Gaynes, B., \& Beil, T. (2009). Screening for depression in adults and older adults in primary care: An updated systematic review. Rockville (MD): Agency for Healthcare Research and Quality. Available from: http://www.ncbi.nlm.nih.gov/books/NBK36406/

Oslin, D., Grantham, S., Coakley, E., Maxwell, J., Miles, K., Ware, J., Blow, F., Krahn, D., Bartels, S., Zubritsky, C., Olsen, E., Kirchner, J., \& Levkoff, S. (2006). PRISM-E: Comparison of integrated care and enhanced specialty referral in managing at-risk alcohol use. Psychiatric Services, 57(7), 954-958.

Park, J.H., Lee, S.B., Lee, T.J., Lee, D.Y., Jhoo, J.H., Youn, J.C., Choo, I.H., Choi, E.A., Jeong J.W., Choe, J.Y., Woo J.I., \& Kim K.W. (2007). Depression in vascular dementia is quantitatively and qualitatively different from depression in Alzheimer's disease. Dementia and Geriatric Cognitive Disorders, 23(2), 67-73. doi: $10.1159 / 000097039$

Parker, G. (2000). Classifying depression: Should paradigms lost be regained? American Journal of Psychiatry, 157(8), 1195-1203.

Parker, G., Roy, K., Hadzi-Pavlovic, D., Wilhelm, K., \& Mitchell, P. (2001). The differential impact of age on the phenomenology of melancholia. Psychological Medicine, 31(7), 1231-1236.

Patient Protection and Affordable Care Act, Pub. L. No. 111-148, § 2703, 124 Stat. 119 (2010). 
Paykel, E., Myers, J., Lindenthal, J., \& Tanner, J. (1974). Suicidal feelings in the general population: A prevalence study. British Journal of Psychiatry, 124, 460469. doi:10.1192/bjp.124.5.460

Pearson, E. \& Hartley, H. (1954). Biometrika tables for statisticians. Cambridge, Massachusetts: Cambridge University Press.

Prigerson, H.G., Frank, E., Kasl, S.V., Reynolds, C.F., Anderson, B., Zubenko, G.S., Houck, P.R., George, C.J., \& Kupfer, D.J. (1995). Complicated grief and bereavement-related depression as distinct disorders: Preliminary empirical validation in elderly bereaved spouses. American Journal of Psychiatry, 152(1), $22-30$.

Proctor, E., Hasche, L., Morrow-Howell, N., Shumway, M., \& Snell, G. (2008). Perceptions about competing psychosocial problems and treatment priorities among older adults with depression. Psychiatric Services, 59 (6), 670-675. doi: 10.1176/appi.ps.59.6.670

Radloff, L. S. (1977). The CES-D scale: A self report depression scale for research in the general population. Applied Psychological Measurements, 1, 385-401.

Rahe, R., Biersner, R., Ryman, D., \& Arthur, R. (1972). Psychosocial predictors of illness behavior and failure in stressful training. Journal of Health \& Social Behavior, 13(4), 393-7. doi: 10.2307/2136831

Rahe, R., Mahan, J., \& Arthur, R. (1970). Prediction of near-future health change from subjects' preceding life changes. Journal of Psychosomatic Research, 14(4), 401406. doi: 10.1016/0022-3999(70)90008-5.

Reijnders, J., Ehrt, U., Weber, W., Aarsland, D., \& Leentjens, A. (2008). A systematic review of prevalence studies of depression in Parkinson's disease. Movement Disorders, 23(2), 183-189.

Rhebergen, D. \& Graham, R. (2014). The re-labelling of dysthymic disorder to persistent depressive disorder in DSM-5: Old wine in new bottles? Current Opinion in Psychiatry, 27(1), 27-21. doi: 10.1097/YCO.0000000000000022.

Roberts, R., Vernon, S. W., \& Rhoades, H. M. (1989). Effects of language and ethnic status on reliability and validity of the CES-D with psychiatric patients. Journal of Nervous and Mental Disease, 177, 581-592.

Robinson, R. \& Price, T. (1982). Post-stroke depressive disorders: A follow-up study of 103 patients. Stroke,13(5), 635-641 
Robinson, D., Geske, J., Prest, L., \& Barnacle, R. (2005). Depression treatment in primary care. Journal of the American Board of Family Medicine. 18(2), 79-86. doi: $10.3122 / \mathrm{jabfm} .18 .2 .79$

Rutledge, T., Vaccarino, V., Johnson, B.D., Olson, M.B., Cornell, C.E., Sheps, D.S., Francis, J., Krantz, D.S., Bairey Merz, C.N., Parashar, S., Handberg, E., Vido, D.A., \& Shaw, L.J. (2009). Depression and cardiovascular healthcarecosts among women with suspected myocardial ischemia: prospective results from the WISE (women's ischemia syndrome evaluation) study. Journal of the American College of Cardiology. 53(2), 176-183. doi: 10.1016/j.jacc.2008.09.032.

Rutter, M. (1985). Resilience in the face of adversity: Protective factors and resistance to psychiatric disorder. British Journal of Psychiatry, 147, 598-611. doi: 10.1192/bjp.147.6.598

Rutter, M. (1979). Protective factors in children's responses to stress and disadvantage. In M.W. Kent \& J.E. Rolf (Eds.). Primary Prevention of Psychopathology (pp.4974).

Sahyoun, N., Pratt, L., Lentzner, H., Dey, A., \& Robinson, K. (2001). The changing profile of nursing home residents: 1985-1997. Aging Trends, 4, 1-8.

Santrock, J. (2011). Life-span development (13th ed.). New York: McGraw-Hill.

Saleebey, D. 1996. The strengths perspective in social work practice: Extensions and cautions. Social Work, 41(3), 296-306.

Scambler, G. (2008). Sociology as applied to medicine (6th ed). Oxford: Saunders Elsevier.

Schaie, K. W., \& Willis, S. (Eds.). (2011). Handbook of the psychology of aging (7th ed.). New York: Academic Press.

Schonfeld, L., King-Kallimanis, B., Duchene, D., Etheridge, R., Herrera, J., Barry, K., \& Lynn, N. (2010). Screening and brief intervention for substance misuse among older adults: the Florida BRITE project. American Journal of Public Health, 100 (1), 108-114. doi: 10.2105/AJPH.2008.149534.

Sheehan, D., Lecrubier, Y., Sheehan, K., Amorim, P., Janavs, J., Weiller, E., Hergueta, T., Baker, R., \& Dunbar, C. (1998). The Mini-International Neuropsychiatric Interview (M.I.N.I.): The development and validation of a structured diagnostic psychiatric interview for DSM-V and ICD-10. Journal of Clinical Psychiatry, 59 (Suppl. 20), 22-33.

Shin, J. (2009). Application of Repeated-Measures Analysis of Variance and Hierarchical Linear Model in Nursing Research. Nursing Research, 3, 211-217. doi: 10.1097/NNR.0b013e318199b5ae 
Shrout, P.E. \& Fleiss, J.L. (1979) Intraclass correlations: Uses in assessing rater reliability. Psychological Bulletin, 2, 420-428.

Simon, G.E., VonKorff, M., \& Barlow, W. (1995). Healthcarecosts of primary care patients with recognized depression. Archives of General Psychiatry. 52(10), 850856.

Smith, T. F. \& Hirdes, J. P. (2009). Predicting social isolation among geriatric psychiatry patients. International Psychogeriatrics, 21, 50-59. doi:10.1017/S1041610208007850.

Snijders, T. (2005). Power and Sample Size in Multilevel Linear Models. In: B.S. Everitt and D.C. Howell (eds.), Encyclopedia of Statistics in Behavioral Science. Volume 3, 1570-1573. Chicester (etc.): Wiley, 2005.

Snijders, T. \& Bosker, R. (1999). Multilevel analysis: An introduction to basic and advanced multilevel modeling. Sage: London.

Sobell, L. C., Sobell, M. B., Leo, G. I., \& Cancilla, A. (1988). Reliability of a timeline method: Assessing normal drinkers' reports of recent drinking and a comparative evaluation across several populations. British Journal of Addiction, 83, 393-402.

Sözeri-Varma, G. (2012). Depression in the elderly: Clinical features and risk factors. Aging and Disease, 3(6), 465-471.

Spitzer, R. L., Williams, J. B., Kroenke, K., Linzer, M., deGruy, F. V., III, Hahn, S. R., Brody, D., \& Johnson, J. (1994). Utility of a new procedure for diagnosing mental disorders in primary care. The PRIME-MD 1000 Study. Journal of the American Medical Association, 272, 1749-1756.

Substance Abuse and Mental Health Services Administration. (2011). The treatment of depression in older adults: Depression and older adults: Key issues. HHS Pub. No. SMA-11-4631, Rockville, MD: Center for Mental Health Services, Substance Abuse and Mental Health Services Administration, U.S. Department of Health and Human Services.

Sullivan, M., LaCroix, A., \& Baum, C. (1997). Functional status in coronary artery disease: A one year prospective study of the role of anxiety and depression. American Journal of Medicine, 103(3), 348-356.

Sullivan, M., Simon, G., Spertus, J., \& Russo, J. (2002). Depression-related costs in heart failure care. Archives of Internal Medicine, 162(16), 1860-66.

Tabachnick, B.G., \& Fidell, L.S. (2012). Using multivariate statistics (6th ed.). Boston: Pearson. 
Tabachnick, B.G., \& Fidell, L.S. (2001). Using multivariate statistics (4th ed.). Boston: Pearson.

Thomas, A. \& O'Brien, J. (2006). Mood disorders in the elderly. Psychiatry, 5(4), 127130. http://dx.doi.org/10.1383/psyt.2006.5.4.127

Umberson, D., Wortman, C., \& Kessler, R. (1992). Widowhood and depression: Explaining long-term gender differences in vulnerability. Journal of Health and Social Behavior, 33(1), 10-24.

U.S. Department of Health and Human Services. (1995). The physician's guide to helping patients with alcohol problems. Washington, DC: Government Printing Office.

U.S. Preventive Services Task Force. (2004). Screening and behavioral counseling interventions in primary care to reduce alcohol misuse: Recommendation statement. Annals of Internal Medicine, 140, 554-556.

Vartiainen, E., Paavola, M., McAlister, A., \& Puska, P. (1998). Fifteen-year follow-up of smoking prevention effects in the North Karelia youth project. American Journal of Public Health, 88(1), 81-85.

Verbrugge, L. (1995). Sex differences in health. In G. Maddox (Ed.), Encyclopedia of aging, 2nd edition (pp. 850-854). New York, NY: Springer Publishing Company.

Vincent, G. \& Velkoff, V. (2010). The next four decades, the older population in the United States: 2010 to 2050. Current Population Reports, Washington, DC.: U.S. Census Bureau.

Waite, A., Bebbington, P., Skelton-Robinson, M. \& Orrell, M. (2004), Social factors and depression in carers of people with dementia. International Journal of Geriatric Psychiatry, 19(6), 582-587. doi: 10.1002/gps.1136

Warr, P., Butcher, V., \& Robertson, I. (2004). Activity and psychological well-being in older people. Aging and Mental Health, 8(2), 172-83.

Webber, S., Porter, M., \& Menec, V. (2010). Mobility in older adults: A comprehensive framework. The Gerontologist, 50(4), 443-450. doi: 10.1093/geront/gnq013

Weisner, C., Mertens, J., Parthasarathy, S., Moore, C.,\&Lu,Y. (2001). Integrating primary medical care with addiction treatment: a randomized controlled trial. Journal of the American Medical Association, 286(14), 1715-1723.

Welch, C.A., Czerwinski, D., Ghimire, B., \& Bertsimas, D. (2009). Depression and costs of health care. Psychosomatics, 50(4), 392-401. doi: 10.1176/appi.psy.50.4.392 
Werner, E. \& Smith, R. (1992). Overcoming the odds: High-risk children from birth to adulthood. New York: Cornell University Press.

Whiteman, K., Ruggiano, N., \& Thomlison, B. (2014). How older women's life experiences influence depression. Unpublished manuscript, Department of Social Welfare, Florida International University, Miami, Florida.

Woltman, H., Feldstain, A., MacKay, C., \& Rocchi, M. (2012). An introduction to hierarchical linear modeling. Tutorials in Quantitative Methods for Psychology, $8(1), 52-69$.

Woon, T., Masuda, M., Wagner, N., \& Holmes, T. (1971). The social readjustment rating scale: A cross-cultural study of Malaysians and Americans. Journal of CrossCultural Psychology, 2(4), 373. doi: 10.1177/002202217100200407

Wormer, K. (2007). Human behavior and the social environment, micro level: Individuals and families. New York: Oxford University Press.

Yesavage, J., Brink, T., Rose, T., Lum, O., Huang, V., Adey, M., \& Leirer, V.O. (1982). Development and validation of a geriatric depression screening scale: A preliminary report. Journal of Psychiatric Research, 17 (1), 37-49.

Zeiss, A. (2005). Depression in long-term care: Contrasting a disease model with attention to environmental impact. Clinical Psychology: Science and Practice, 12(3), 300-2. doi: 10.1093/clipsy.bpi038

Zubenko, G., Hughes, H., Stiffler, J., Brechbiel, A., Zubenko, W., Maher, B., \& Marazita M. (2003). Sequence variations in CREB1 cosegregate with depressive disorders in women. Molecular Psychiatry, 8(6), 611-8. 
Appendix A

12-item General Health Questionnaire (GHQ-12) (Goldberg \& Williams, 1988)

Have you recently...

1. Been able to concentrate on whatever you are doing

2. Lost much sleep over worry

3. Felt that you are playing a useful part in things

4. Felt capable of making decisions about things

5. Felt constantly under strain

6. Felt you couldn't overcome your difficulties

7. Been able to enjoy your normal day to day activities

8. Been able to face up to your problems

9. Been feeling unhappy and depressed

10.Been losing confidence in yourself

11.Been thinking of yourself as a worthless person

12.Been feeling reasonably happy

\begin{tabular}{|c|c|c|c|}
\hline Always & & & Never \\
\hline 0 & 1 & 2 & 3 \\
\hline Never & & & Always \\
\hline 0 & 1 & 2 & 3 \\
\hline Always & & & Never \\
\hline 0 & 1 & 2 & 3 \\
\hline Always & & & Never \\
\hline 0 & 1 & 2 & 3 \\
\hline Never & & & Always \\
\hline 0 & 1 & 2 & 3 \\
\hline Never & & & Always \\
\hline 0 & 1 & 2 & 3 \\
\hline Always & & & Never \\
\hline 0 & 1 & 2 & 3 \\
\hline Always & & & Never \\
\hline 0 & 1 & 2 & 3 \\
\hline Never & & & Always \\
\hline 0 & 1 & 2 & 3 \\
\hline Never & & & Always \\
\hline 0 & 1 & 2 & 3 \\
\hline Never & & & Always \\
\hline 0 & 1 & 2 & 3 \\
\hline Always & & & Never \\
\hline 0 & 1 & 2 & 3 \\
\hline
\end{tabular}

TOTAL GHQ-12 SCORE (0-36)

Scoring higher scores indicate worse health. 
Appendix B

Short Orientation-Memory-Concentration Test (Katzman et al., 1983)

Patient: DATE:

Age:

\section{Short Blessed Test (SBT)1}

"Now I would like to ask you some questions to check your memory and concentration. Some of them may be easy and some of them may be hard."

1. What year is it now?

2. What months is it now?

Please repeat this name and address after me:

John Brown, 42 Market Street, Chicago

John Brown, 42 Market Street, Chicago

John Brown, 42 Market Street, Chicago
Correct Incorrect

Correct Incorrect

(0)

(1) 
(underline words repeated correctly in each trial) Trials to learning (can't do in 3 trials $=\mathrm{C}$ )

Good, now remember that name and address for a few minutes.

3. Without looking at your watch or clock, tell me about what time it is.

(If response is vague, prompt for specific response) Correct Incorrect

(within 1 hour) (0)

(1)

Actual time:

4. Count aloud backwards from 20 to 1 $\begin{array}{llll}0 & 1 & 2 & \text { Errors }\end{array}$ (Mark correctly sequenced numerals) If subject starts counting forward or forgets the task, repeat instructions and score one error

20191817161514131211

10987654321

5. Say the months of the year in reverse order.

If the tester needs to prompt with the last name of the months of the year, one error should be scored (Mark correctly sequenced months) 
6. Repeat the name and address I asked you to remember. (The thoroughfare term (Street) is not required)

\begin{tabular}{lllllllll} 
(John Brown, & 2 Market Street, Chicago) & 0 & 1 & 2 & 3 & 4 & 5 \\
& \multicolumn{8}{c}{ Errors }
\end{tabular}

Check correct items USE ATTACHED SCORING GRID \& NORMS

A spontaneous self-correction is allowed for all responses without counting as an error.

1. What is the year?

Acceptable Response: The exact year must be given. An incomplete but correct numerical response is acceptable (e.g., 01 for 2001).

2. What is the months?

Acceptable Response: The exact months must be given. A correct numerical answer is acceptable (e.g., 12 for December).

3. The clinician should state: "I will give you a name and address to remember for a few minutes. Listen to me say the entire name and address and then repeat it after me." 
It is important for the clinician to carefully read the phrase and give emphasis to each item of the phrase. There should be a one second delay between individual items. The trial phrase should be re-administered until the subject is able to repeat the entire phrase without assistance or until a maximum of three attempts. If the subject is unable to learn the phrase after three attempts, a "C" should be recorded. This indicates the subject could not learn the phrase in three tries. Whether or not the trial phrase is learned, the clinician should instruct "Good, now remember that name and address for a few minutes.”

4. Without looking at your watch or clock, tell me about what time it is? This is scored as correct if the time given is within plus or minus one hour. If the subject's response is vague (e.g., "almost 1 o'clock), they should be prompted to give a more specific response.

5. Counting. The instructions should be read as written. If the subject skips a number after 20, an error should be recorded. If the subject starts counting forward during the task or forgets the task, the instructions should be repeated and one error should be recorded. The maximum number of errors is two.

6. Months. The instructions should be read as written. To get the subject started, the examiner may state "Start with the last months of the year. The last months of the year is__. . If the subject cannot recall the last months of the year, the examiner may prompt this test with "December"; however, one error should be recorded. 
If the subject skips a months, an error should be recorded. If the subject starts saying the months forward upon initiation of the task, the instructions should be repeated and no error recorded. If the subject starts saying the months forward during the task or forgets the task, the instructions should be repeated and one error recorded. The maximum number of errors is two.

7. Repeat. The subject should state each item verbatim. The address number must be exact (i.e. "4200" would be considered an error for " 42 "). For the name of the street (i.e. Market Street), the thoroughfare

term is not required to be given (ie. Leaving off "drive" or "street") or to be correct (ie. Substituting "boulevard" or lane") for the item to be scored correct.

8. The final score is a weighted sum of individual error scores. Use the table on the next page to calculate each weighted score and sum for the total.

These guidelines and scoring rules are based on the administration experience of faculty and staff of the Memory and Aging Project, Alzheimer's Disease Research Center, Washington University School of Medicine, St. Louis (John C. Morris, MD, Director \& PI; morrisj@abraxas.wustl.edu).For more information about the ADRC, please visit our website: http://alzheimer.wustl.edu or call 314-286-2881.

\section{$\underline{\text { Final SBT Score \& Interpretation }}$}


Item Errors (0 - 5) Weighting Factor Final Item Score

\begin{tabular}{|l|l|l|l|}
\hline Item & Errors (0 - 5) & Weighting Factor & Final Item Score \\
\hline 1 & & $\mathrm{X} 4$ & \\
\hline 2 & & $\mathrm{X} 3$ & \\
\hline 3 & & $\mathrm{X} 3$ & \\
\hline 4 & & $\mathrm{X} 2$ & \\
\hline 5 & & $\mathrm{X} 2$ & \\
\hline 6 & & $\mathrm{X} 2$ & \\
\hline & & & Sum Total $=$ \\
& & & (Range 0 - 28) \\
\hline
\end{tabular}

\section{$\underline{\text { Interpretation }}$}

A screening test in itself is insufficient to diagnose a dementing disorder. The SBT is, however, quite sensitive to early cognitive changes associated with Alzheimer's disease. Scores in the impaired range (see below) indicate a need for further assessment. Scores in the "normal" range suggest that a dementing disorder is unlikely, but a very early disease process cannot be ruled out. More advanced assessment may be warranted in cases where other objective evidence of impairment exists. $f$ In the original validation sample for the SBT (Katzman et al., 1983), 90\% of normal scores 6 points or less. Scores of 7 or higher would indicate a need for further evaluation to rule out a dementing disorder, such as Alzheimer's disease. $f$ Based on clinical research findings from the Memory and Aging Project, the following cut points may also be considered:

- 0 -4 Normal Cognition 
- 5-9 Questionable Impairment (evaluate for early dementing disorder)

- 10 or more Impairment Consistent with Dementia (evaluate for dementing disorder) 


\title{
Appendix C
}

\section{Letter of Agreement}

\author{
UNIVERSITY OF PENNSYLVANIA HEALTH SYSTEM \& \\ UNIVERSITY OF PENNSYLVANIA SCHOOL OF MEDICINE
}

DATA USE AGREEMENT

This Data Use Agreement ("Agreement") is made and entered into as of this 28 day offuly, 2013 by and between the Iniversity of Pennsylvania Health System (UPHS) and the University of Pennsylvania School of Medicine (SOM) ("Covered Fintity") and Karen Fortuna ("Data Recipient").

1. This Agreement sets forth the terms and conditions pursuant to which Covered Entity will Disclose certain Protected Health Information (PHI) to the Data Recipient as described below (insert a meaningful description of the data set):

Primary Care Research in Substance Abuse and Mental Health for Elderly (PRISM-E) study Except as otherwise specified herein. Data Recipient may make Uses and Disclosures of the Limited Data Set consistent with the purpose of the research as described in the research application. The title of the research project has been provided below:

Gender-based Differences in Longitudinal Depression Treatment Outcomes Among Older Primary Care Patients

3. In addition to the Data Recipient, the individuals, or classes of individuals, who are permitted to Use or receive the Limited Data Set for purposes of the Research Project, include:

Kiaren Fortuna

4. Data Recipient agrees to not Use or Disclose the Limited Data Set for any purpose other than the Research Project or as Required by Law.

5 Data Recipient agrees to use appropriate safeguards to prevent Use or Disclosure of the Limited Data Set other than as provided for by this Agreement.

6. Data Recipient agrees to report to the Covered Entity any Use or Disclosure of the Limited Data Set not Druse provided for by this Agreement, of which within ten (10) days of its discovery.

Dat Recipient agrees to ensure that any agent, including a subcontractor, to whom it provides the limited the same restrictions and conditions that apply through this Agreement to the Data ct to such information.

8. Data Recipient agrees not to identify the information contained in the Limited Data Set or comtact the individual.

Data Recipient will indemnify. defend and hold harmless Covered Entity and any of covered Entity"s Trive trustees, officers, directors, employees and agents ("Indemnitees") from and affiliates, and their respective trustion, liability, damage. cost or expense (including without limitation. against any claim, cause of action, liabilits arising out of or in connection with any unauthorized or reasonable attorney"s fees and court costs) arising out of or in connection wh the prohibieg Use or Disclosure of the Limited Data Set or any other breach

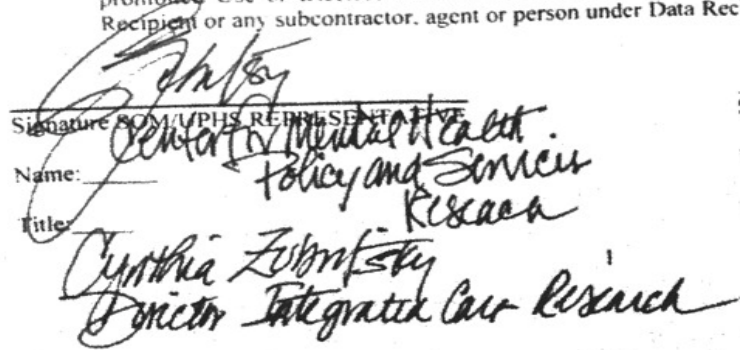

Name:Karen Fortuna

Title:Mrs. 


\section{Appendix D}

\section{General Questionnaire}

\section{A. SAMHSA Demographics}

"First we'd like to start with a few questions about your background."

A1. What is the highest level of school you have completed?
$O$ Less than 8 th grade
O Some college or trade school
Don't know / Refused
Less than 12 th grade
College graduate
High school graduate/GED
Graduate schoo

A2. What country were you born in?

O United States

Other Specify (PLEASE PRINT):

O Don't know / Refused

A2a. (IF COUNTRY OF BIRTH IS NOT THE U.S.) How many years have you lived in the United States?
O Less than 1 year
6-9 Years
1-5 Years
10 or more years
Don't know / Refused

A3. Do you have any (surviving) children?
O Yes
$\rightarrow \quad \rightarrow \quad$ (IF YES) How many?
O No
Don't know / Refused

A4. How many close friends do you have? (People that you feel at ease with, can talk to about private matters, and call on for help.)
None
01 or 2
3 to 5
O 6 to 9 O 10 or more
O Don't know / Refused

A5. How many relatives do you have that you feel close to?
O None
or 2
03 to 5
06 to 9
10 or more
Don't know / Relused

A6. How many of these friends and relatives do you see at least once a month?
O None
O 1 or 2
3 to 5
06 to 9
10 or more
O Dont know / Refused

A7. What is your religious preference?
Protestant
Jewish
Buddhist
None
O Roman Catholic
O Islam
Other
O Don't know / Refused

A8. How often do you participate in religious or spiritual activities?
O Daily
Weekly
O Monthly
On special occasions
O Never 
A9. Do you belong to any of these kinds of groups?

$\begin{array}{lcc}\text { a. Social or recreational group } & \text { Yes } & \text { No } \\ \text { b. Church group } & 0 & 0 \\ \text { c. Labor union, commercial or professional organization } & 0 & 0 \\ \text { d. A group concerned with children (PTA, Boy Scouts) } & 0 & 0 \\ \text { e. Charity group } & 0 & 0 \\ \text { f. Other group } & 0 & 0\end{array}$

Specify (PLEASE PRINT):

A10. In your life as a whole, what has been your main occupation?

(PLEASE PRINT):

A11. Are you currently doing any kind of work that you are being paid for?
O Yes, part time
Yes, full time
No
O Don't know / Refused

A12. Do you do volunteer work?

OYes O No

A13. Thinking about your financial situation, would you say you :
Can't make ends meet
Are comfortable
Have just enough to get along
Don't know / Refused

$\prod \rightarrow \frac{3}{3}$

ASK TO SEE INSURANCE CARD(S). RECORD THE MEDICARE CLAIM NUMBER FROM THE MEDICARE CARD. USE CARDS TO ANSWER A14 AND A15.

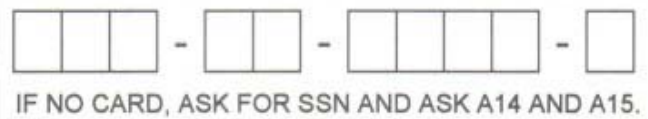

A14. Do you receive Medicare benefits? (Medicare is a federal program to provide health insurance for older adults.)
O Yes
O No
Don't know / Refused

A14a. (IF YES) is your Medicare insurance provided by a managed care plan? (HMO or Preferred Provider)

$$
\text { Yes O No ODon't know/Refused }
$$

A15. Do you receive Medicaid benefits? (Medicaid is a state program to provide health insurance for people who can't afford insurance.)

O Yes O No O Don't know/Refused

A15a. (IF YES) Is your Medicaid Insurance provided by a managed care plan? (HMO or Preferred Provider)

OYes O No ODon't know/Refused

\section{8}


A16. Are you eligible for VA benefits?

OYes ONo O Don't know/Refused

A16a. (IF YES) What percent benefits are you ellgible for?

$$
\text { O } 100 \% \quad \text { O 50-99\% O }<50 \% \text { O Don't know / Refused }
$$

A17. Do you have any medical insurance other than Medicaid, Medicare, or VA benefits? (PROBE: Sometimes called Medigap insurance.)

OYes ONo O Don't know / Refused

A17a. (IF YES) What is the name of this insurance?

A18. Do you have prescription drug coverage, either through your health insurance or through a special government program?

OYes ONo O Don't know/Refused

A18a. (IF YES) How much do you pay when you get a prescription medicine? (FILL ALL THAT APPLY)

$$
\begin{array}{ll}
\text { Nothing } & \text { Between } \$ 2 \text { and } \$ 10 \\
\text { Less than } \$ 2 & \text { O More than } \$ 10 \\
\text { \$2 } & \text { O Depends on the medicine }
\end{array}
$$

A19. Are you currently recelving formal mental health (not substance abuse) treatm ent from a mental health provider such as a counselor, psychiatrist, therapist or social worker?

O Yes O No

A20. Are you currently recelving formal alcohol treatment from a substance abuse provider such as a substance abuse counselor, or psychiatrist?

OYes ONo

CHECKPOINT I
Record A19 AND A20 responses on worksheet.
If YES to A19 AND A20, consumer is ineligible for study. Thank them
for their participation and conclude interview.
Otherwise, Continue.




\section{P. MINI Alcohol Target Condition}

"In this next section, I am going to ask you some questions concerning your use of alcoholic beverages like beer, wine, wine coolers, or hard liquor like vodka, gin, or whiskey."

P1. Have you had a drink of alcohol in the past year?

$\begin{array}{cc}\text { YES } & \text { NO } \\ O & 0\end{array}$

If OTHERWISE, CONTINUE.

P2. During the last week, did you have any drink containing alcohol?

OYes

O No (SKIP TO P4.)

"Now I'm going to ask you about the number of alcoholic drinks you've had each day in the last week. Today is (NAME DAY OF WEEK). Let's begin with yesterday."

P3. How many drinks of beer, wine, or liquor did you have on (NAME DAY OF WEEK)?

\begin{tabular}{|l|l|l|l|l|l|l|l|l|}
\hline \multicolumn{3}{|c|}{ Sunday } & \multicolumn{1}{c|}{ Monday } & Tuesday & Wednesday & \multicolumn{2}{c|}{ Thursday } & \multicolumn{2}{c|}{ Friday } \\
\hline $\begin{array}{l}\text { Number } \\
\text { of drinks }\end{array}$ & & & & & & & & \\
\hline
\end{tabular}

TI DAY, THEN ASK FOR THE NUMBER OF DRINKS DRUNK ON A TYPICAL DAY. RECORD THIS VALUE IN THE BOXES FOR EACH DAY THE CONSUMER WAS UNABLE TO REPORT ALCOHOL CONSUMPTION.

Note:

- A single mixed drink

- A glass of wine $(5 \mathrm{oz})$

$=1$ drink

- A wine cooler (12 oz)

$=1$ drink

- A 12 oz beer

$=1.5$ drinks

- A shot of hard liquor(1.5 oz)

$=1 \mathrm{drink}$

- A pitcher of beer

$=1$ drink

- A pint of hard liquor

$=4$ drinks

- A fifth of hard liquor

$=11$ drinks

- A pint of wine

$=18$ drinks

- A bottle of wine(40 oz)

$=3$ drinks

$=8$ drinks

P4. During the last three months, on days that you drank any alcohol, how many times did you drink four or more drinks in one day?

Total number of binges: 


\section{ALCOHOL TARGET CONDITION DLAGNOSIS \#1}

RULE 1 A: IS TOTAL NUMBER DRINKS >= 14 AND MALE?

B: IS TOTAL NUMBER OF DRINKS $>=12$ AND FEMALE?

RULE 2: IS TOTAL NUMBER OF BINGES $>=4$ ?

IF EITHER RULE 1 = YES OR RULE 2 = YES, GO TO DIAGNOSIS BOX AND CIRCIE VES FOR AT RISK DRINKING.

OTHERWISE, CONTINUE TO NEXT RULE BOX.

\section{ALCOHOL TARGET CONDITION DIAGNOSIS $\approx 2$}

RULE 1: QUALIFY FOR ALCOHOL-TARGET MED (p8)?

RULE 2: IS TOTAL NUMGER OF DRINKS >= 7 ?

YES

N UNSURE

IF RULE 1 = YES AND RULE 2 = YES, GO TO DIAGNOSIS BOX AND CRCA A IEI FUR AT RISK DRINKNG

IF EITHER RULE $1=$ NO OR RULE $2=N O, G O$ TO DIAGNOSIS BOX AIC I=R= = NO ROR AT RISK DRANKNG.

IF RULE 1 = UNSURE ANO RULE 2 = YES, CIRCLE UNSURE. 


\section{S. Baseline Multicultural Assessment}

"We have been talking for awhile about how you have been feeling. Now l'd like to switch gears a little and talk more generally about your attitudes towards mental health and alcohol use."

S1. Who makes most of the decisions about your health care? (READ OPTIONS and FILL ALL THAT APPLY)

OYou

O family member other than your spouse

O Your spouse or significant other

O Someone else

O Your doctor

O Don't know / Refused

READ THE NEXT QUESTION AS IF IT WAS OPEN ENDED. IF THE CONSUMER CAN'T PROVIDE AN ANSWER, THEN SHOW CARD C AND CHECK HERE: $\quad \square$ Card shown

S2. Who would you talk to if you had a mental health or alcohol problem? (PROBE: I don't know if you have a problem, but if you were to ever have a problem, who would you talk to?) (FILL ALL THAT APPLY)

O Your spouse or significant other

O A family member who lives with you other than your spouse

O A family member who doesn't live with you

$O A$ friend

O A healer in the community

O A psychiatrist

O A medical doctor

O A social worker

O A psychologist

O A 12-step program

O Someone from a church, synagogue or other religious group

O A spiritual leader not associated with any organized religion

O Alternative care provider (acupuncture, massage, etc)

O No one

O Other Specify (PLEASE PRINT):

O Don't know / Refused

S3. If you had a mental health or alcohol abuse problem, what do you think would help you to get better? (READ OPTIONS and FILL ALL THAT APPLY)

O Pills or medication

Herbal remedies

Private counseling

Group counseling
Alternative therapies (acupuncture, massage, spiritualism)

Spiritual advice

Other specify:

Don't know / Refused

\section{8}


II READ THE NEXT 2 QUESTIONS AS IF THEY WERE OPEN ENDED. IF THE CONSUMER CAN'T PROVIDE AN ANSWER, THEN SHOW CARD D AND CHECK HERE: $\square$ Card shown

S4. Nobody knows for sure what causes mental health problems such as depression, but people have many different ideas about what the causes might be. What do you think causes depression? (FILL ALL THAT APPLY)

Stressl O Loss (eg. family, friends)

Loss: $\quad$ Loss or lack of pleasurable activities, work

O Family issues

Money issues

Political stress

Safety issues

O Stress or worry (general)

Medical: O Medical illness

Infectious disease (germs)

Nutritional deficiency

Chemical imbalance

$O$ Genetic (inherited) disorder

Spirit/ Disturbance in harmony of body, mind and spirit/ negative thinking

psyche: $\quad$ Something you did wrong in the past

O Supernatural causes (witchcraft, evil eye, hexes, spiritual forces, spiritual possession)

Envirl O Moving to a different place

Culture: $\quad$ Cultural differences

Adjusting to a different culture

O Don't know / Refused

O Other Specify (PLEASE PRINT): 
S5. What do you think are the causes for alcohol problems? (FILL ALL THAT APPLY)

Stress/ O Loss (eg. family, friends)

Loss: $\quad$ Loss or lack of pleasurable activities, work

O Family issues

Money issues

O Political stress

O Safety issues

O Stress or worry (general)

Medical: O Medical illness

Infectious disease (germs)

O Nutritional deficiency

O Chemical imbalance

Genetic (inherited) disorder

Spirit/ Disturbance in harmony of body, mind and spirit/ negative thinking

psyche: O Something you did wrong in the past

O Supernatural causes (witchcraft, evil eye, hexes, spiritual forces, spiritual possession)

Envirl O Moving to a different place

Culture:

Cultural differences

Adjusting to a different culture

O Don't know / Refused

O Other Specify (PLEASE PRINT):

S6. In the treatment of health problems, how important is it for your health care provider to ... (REFER TO CARD E)

Not at all Somewhat Moderately Quite Very

Important Important

Important Important

a. Speak your native language?

$\mathrm{O}$

o

O

0

b. Belong to the same racial or ethnic group?

0

0

0

O

c. Be of the same gender as you?

○

0

0

$\mathrm{O}$

d. Be the same age as you?

O

O

$\mathrm{O}$

O

e. Be open to different treatments such as acupuncture, massage, spiritualism, nutritional supplements,etc?

f. Understand your culture?

0

0

0

O

0

$\mathrm{O}$

0

0

0


Appendix E

Medical History Checklist

BASELINE ASSESSMENT VARIABLES: Medical History Checklist (Yes(1)/No(2))

Diabetes or sugar sickness

High Blood pressure or hypertension

Heart trouble

circulation problems, hardening

Been paralyzed in any way

Any other effects of stroke

Arthritis, rheumatism

A stomach ulcer

Emphysema or asthma

Glaucoma, pressure behind the eye

Cataracts

Liver trouble or jaundice

Gall bladder trouble

Kidney trouble

Bladder trouble

A broken hip

Other broken bones

Anemia

Parkinsons disease 
Sleep trouble

Skin trouble

Cancer 


\title{
Appendix F
}

\section{The Social Readjustment Rating Scale (Holmes-Rahe, 1967)}

\author{
Life event Life change units
}

Death of a spouse

100

Divorce

73

Marital separation

65

Imprisonment

63

Death of a close family member

63

Personal injury or illness

53

Marriage

50

Dismissal from work

47

Marital reconciliation

45

Retirement

45 
Life event Life change units

Change in health of family member $\quad 44$

Pregnancy $\quad 40$

Sexual difficulties $\quad 39$

Gain a new family member $\quad 39$

Business readjustment $\quad 39$

Change in financial state $\quad 38$

Death of a close friend $\quad 37$

Change to different line of work $\quad 36$

Change in frequency of arguments $\quad 35$

Major mortgage $\quad 32$

Foreclosure of mortgage or loan $\quad 30$

Change in responsibilities at work 29

Child leaving home $\quad 29$ 
Life event Life change units

Trouble with in-laws

29

Outstanding personal achievement

28

Spouse starts or stops work

26

Begin or end school

26

Change in living conditions

25

Revision of personal habits

24

Trouble with boss

23

Change in working hours or conditions 20

Change in residence

20

Change in schools

20

Change in recreation

19

Change in church activities

19

Change in social activities

18 
Life event Life change units

Minor mortgage or loan

17

Change in sleeping habits

16

Change in number of family reunions

15

Change in eating habits

15

Vacation

13

Christmas

12

Minor violation of law

11

Score of 300+: At risk of illness.

Score of 150-299: Risk of illness is moderate (reduced by 30\% from the above risk). Score $<150$ : Only have a slight risk of illness. 


\section{Appendix G}

Alcohol Consumption (Sobell, Sobell, Leo, \& Cancilla, 1988).

1. Have you had a drink of alcohol past year? Yes or No

2. Have you had a drink of alcohol past Week? Yes or No

3. How many alcohol drinks have you had in the past week? 


\section{Appendix $\mathrm{H}$}

Center for Epidemiologic Studies Depression Scale (CES-D) (Radloff, 1977)

Below is a list of the ways you might have felt or behaved.

Please tell me how often you have felt this way during the past week.

$\begin{array}{llll}\begin{array}{l}\text { Rarely or } \\ \text { none of }\end{array} & \begin{array}{l}\text { Some } \\ \text { or a }\end{array} & \begin{array}{l}\text { Occasionally } \\ \text { or a }\end{array} & \begin{array}{l}\text { Most or } \\ \text { all of the }\end{array} \\ \text { the time } & \text { little } & \text { moderate } & \text { time (5-7 } \\ \text { (less than } & \text { time (1- } & \text { amount of } & \text { days) } \\ \text { one day) } & 2 \text { days) } & \text { time (3-4 } & \end{array}$

1. Lwas botherest by things that usually. dop'thotherses.

2. I did not feel like eating; my appetite was poor.

3. I felt that I could not shake off the blues even with help from my family or friends.

4. I felt I was just as good as other people.

5. I had trouble keeping my mind on what I was doing.

6. I felt depressed.

7. I felt that everything I did was an effort.

8. I felt hopeful about the future.

9. I thought my life had been a failure.

10. I felt fearful.

11. My sleep was restless.

12. I was happy.

13. I talked less than usual.

14. I felt lonely.

15. People were unfriendly.

16. I enjoyed life.

17. I had crying spells.

18. I felt sad.

19. I felt that people dislike me.

20. I could not get "going."

days)

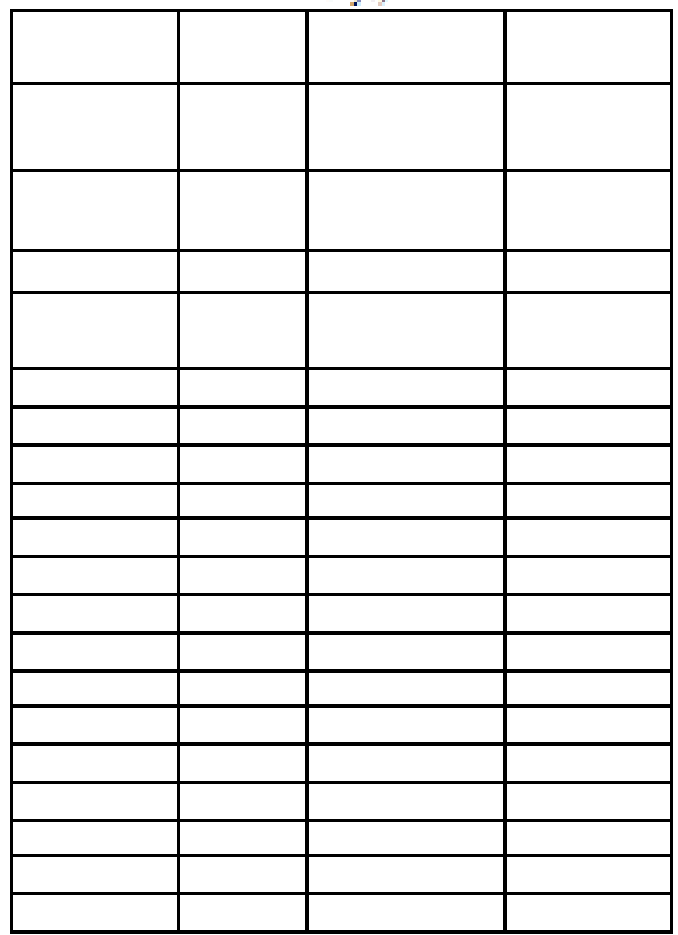

SCORING: zero for answers in the first column, 1 for answers in the socond column, 2 for answers in the third column, 3 for answers in the fourth column. The scoring of positive items is reversed. Possible range of scores is zero to 60 , with the higher scores indicating the presence of more symptomatology. 


\section{Appendix I}

\section{The Paykel Suicide Questionnaire}

1. Has there been a time in the last year that you felt life was not worth living?

2. Has there been a time in the last year that you wished you were dead-for instance, that you could go to sleep and not wake up?

3. Has there been a time in the last year that you thought of taking your own life, even if you would not really do it?

4. Has there been a time in the last year when you have reached the point where you seriously considered taking your life, or perhaps made plans how you would go about doing it?

5. In the last year have you made an attempt on your life?

Scoring: Suicidal ideation is rated as present with a response of "yes" to questions 3,4 , and/or 5 
MINI International Neuropsychiatric Interview (Sheehan et al., 1998)

M.I.N.I.

MINI INTERNATIONAL NEUROPSYCHIATRIC INTERVIEW

\author{
English Version 5.0.0
}

DSM-IV

USA: D. Sheehan, J. Janavs, R. Baker, K. Harnett-Sheehan, E. Knapp, M. Sheehan

University of South Florida - Tampa

FRANCE: Y. Lecrubier, E. Weiller, T. Hergueta, P. Amorim, L. I.

Bonora, J. P. Lépine Hôpital de la Salpétrière - Paris

(c) Copyright 1992-2006 Sheehan DV \& Lecrubier Y

All rights reserved. No part of this document may be reproduced or transmitted in any form, or by any means, electronic or mechanical, including photocopying, or by any information storage or retrieval system, without permission in writing from Dr. Sheehan or Dr. Lecrubier. Researchers and clinicians working in nonprofit or publicly owned settings (including universities, nonprofit hospitals, and government institutions) 


\section{may make copies of a M.I.N.I. instrument for their own clinical and research use.}

\section{DISCLAIMER}

Our aim is to assist in the assessment and tracking of patients with greater efficiency and accuracy. Before action is taken on any data collected and processed by this program, it should be reviewed and interpreted by a licensed clinician.

This program is not designed or intended to be used in the place of a full medical and psychiatric evaluation by a qualified licensed physician psychiatrist. It is intended only as a tool to facilitate accurate data collection and processing of symptoms elicited by trained personnel.

\section{GENERAL INSTRUCTIONS}

The M.I.N.I. was designed as a brief structured interview for the major Axis I psychiatric disorders in DSM-IV and ICD-10. Validation and reliability studies have been done comparing the M.I.N.I. to the SCID-P for DSM-III -R and the CIDI (a structured interview developed by the World Health Organization for lay interviewers for ICD-10). The results of these studies show that the M.I.N.I. has acceptably high validation and reliability scores, but can be administered in a much shorter period of time (mean $18.7 \pm 11.6$ minutes, median 15 minutes) than the above reference groupd instruments. It can be used by clinicians, after a brief training session. Lay interviewers require more extensive training.

\section{INTERVIEW:}

In order to keep the interview as brief as possible, inform the patient that you will conduct a clinical interview that is more structured than usual, with very precise questions about psychological problems which require a yes or no answer.

\section{GENERAL FORMAT:}

The M.I.N.I. is divided into modules identified by letters, each corresponding to a diagnostic category.

-At the beginning of each diagnostic module (except for psychotic disorders module), screening question(s) corresponding to the main criteria of the disorder are presented in a gray box.

-At the end of each module, diagnostic box(es) permit the clinician to indicate whether diagnostic criteria are met.

\section{CONVENTIONS:}

Sentences written in « normal font » should be read exactly as written 
to the patient in order to standardize the assessment of diagnostic criteria.

Sentences written in « CAPITALS » should not be read to the patient. They are instructions for the interviewer to assist in the scoring of the diagnostic algorithms.

Sentences written in « bold» indicate the time frame being investigated. The interviewer should read them as often as necessary. Only symptoms occurring during the time frame indicated should be considered in scoring the responses.

Answers with an arrow above them ( ) indicate that one of the criteria necessary for the diagnosis(es) is not met. In this case, the interviewer should go to the end of the module, circle « NO » in all the diagnostic boxes and move to the next module.

When terms are separated by a slash (/) the interviewer should read only those symptoms known to be present in the patient (for example, question H6).

Phrases in (parentheses) are clinical examples of the symptom. These may be read to the patient to clarify the question.

\section{RATING INSTRUCTIONS:}

All questions must be rated. The rating is done at the right of each question by circling either Yes or No. Clinical judgment by the rater should be used in coding the responses. The rater should ask for examples when necessary, to ensure accurate coding. The patient should be encouraged to ask for clarification on any question that is not absolutely clear.

The clinician should be sure that each dimension of the question is taken into account by the patient (for example, time frame, frequency, severity, and/or alternatives).

Symptoms better accounted for by an organic cause or by the use of alcohol or drugs should not be coded positive in the M.I.N.I. The M.I.N.I. Plus has questions that investigate these issues.

For any questions, suggestions, need for a training session, or information about updates of the M.I.N.I., please contact : 
David V Sheehan, M.D., M.B.A.

Yves Lecrubier, M.D. / Thierry Hergueta, M.S.

University of South Florida College of Medicine 3515 East Fletcher Avenue

INSERM U302

Salpétrière

Tampa, FL USA 33613-4788

Hôpital de la

1'Hôpital

tel : +1 813974 4544; fax : +1 8139744575

47, boulevard de

FRANCE

e-mail :dsheehan@hsc.usf.edu

F. 75651 PARIS,

1616 59; fax : +33 (0) 145852800

tel : $+33(0) 142$

e-mail :

hergueta@ext.juss

ieu.fr

M.I.N.I. 5.0.0 (July 1, 2006)

A. MAJOR DEPRESSIVE EPISODE

( MEANS : GO TO THE DIAGNOSTIC BOXES, CIRCLE NO IN ALL DIAGNOSTIC BOXES, AND MOVE TO THE NEXT MODULE)

\begin{tabular}{|c|c|c|c|}
\hline \multirow[t]{2}{*}{ A1 } & $\begin{array}{l}\text { Have you been consistently depressed or down, } \\
\text { most of the day, nearly }\end{array}$ & $\mathrm{NO}$ & YES \\
\hline & every day, for the past two weeks? & & \\
\hline \multirow[t]{3}{*}{ A 2} & $\begin{array}{l}\text { In the past two weeks, have you been much less } \\
\text { interested in most things or }\end{array}$ & $\mathrm{NO}$ & YES \\
\hline & $\begin{array}{l}\text { much less able to enjoy the things you used to enjoy } \\
\text { most of the time? }\end{array}$ & & \\
\hline & IS A1 OR A2 CODED YES? & $\mathrm{NO}$ & YES \\
\hline \multirow[t]{9}{*}{ A3 } & $\begin{array}{l}\text { Over the past two weeks, when you felt depressed } \\
\text { or uninterested: }\end{array}$ & & \\
\hline & $\begin{array}{l}\text { Was your appetite decreased or increased nearly } \\
\text { every day? Did your weight }\end{array}$ & $\mathrm{NO}$ & YES * \\
\hline & $\begin{array}{l}\text { decrease or increase without trying intentionally } \\
\text { (i.e., by } \pm 5 \% \text { of body weight }\end{array}$ & & \\
\hline & $\begin{array}{l}\text { or } \pm 8 \text { lbs. or } \pm 3.5 \mathrm{kgs} ., \text { for a } 160 \mathrm{lb} . / 70 \mathrm{~kg} \text {. person in } \\
\text { a months)? }\end{array}$ & & \\
\hline & IF YES TO EITHER, CODE YES. & & \\
\hline & $\begin{array}{l}\text { Did you have trouble sleeping nearly every night } \\
\text { (difficulty falling asleep, waking up }\end{array}$ & $\mathrm{NO}$ & YES \\
\hline & $\begin{array}{l}\text { in the middle of the night, early morning wakening } \\
\text { or sleeping excessively)? }\end{array}$ & & \\
\hline & $\begin{array}{l}\text { Did you talk or move more slowly than normal or } \\
\text { were you fidgety, restless }\end{array}$ & $\mathrm{NO}$ & YES * \\
\hline & or having trouble sitting still almost every day? & & \\
\hline
\end{tabular}




\begin{tabular}{|l|l|c|c|}
\hline $\begin{array}{l}\text { Did you feel tired or without energy almost every } \\
\text { day? }\end{array}$ & NO & YES \\
\hline $\begin{array}{l}\text { Did you feel worthless or guilty almost every day? } \\
\begin{array}{l}\text { Did you have difficulty concentrating or making } \\
\text { decisions almost every day? }\end{array}\end{array}$ & NO & YES \\
\hline $\begin{array}{l}\text { Did you repeatedly consider hurting yourself, feel } \\
\text { suicidal, or wish that you were dead? }\end{array}$ & YES \\
\hline & NO & YES \\
\hline & $\begin{array}{l}\text { N } \\
\text { ORE 5 OR MORE ANSWERS (A1-A3) CODED }\end{array}$ & YES * \\
\hline YES? & & \\
\hline
\end{tabular}

*If yes, patient has Major Depressive Episode, Current

IF PATIENT HAS CURRENT MAJOR DEPRESSIVE EPISODE CONTINUE

TO A4.

OTHERWISE MOVE TO MODULE B:

A4 a During your lifetime, did you have other episodes of two weeks or more when you

felt

depressed or uninterested in most things, and had most of the problems we just talked about?

NO

b In between 2 episodes of depression, did you ever have an interval of at least 2 months, without any depression and any loss of interest?

NO

If Yes, to A4a and A4b patient has Major Depressive Episode, recurrent

\section{B. DYSTHYMIA}


(MEANS : GO TO THE DIAGNOSTIC BOX, CIRCLE NO,

AND MOVE TO THE NEXT MODULE)

IF PATIENT'S SYMPTOMS CURRENTLY MEET CRITERIA FOR MAJOR

DEPRESSIVE EPISODE, DO NOT EXPLORE THIS MODULE.

\begin{tabular}{|l|l|l|l|}
\hline B1 & $\begin{array}{l}\text { Have you felt sad, low or depressed most of } \\
\text { the time for the last two years? }\end{array}$ & NO & YES \\
\hline B2 & $\begin{array}{l}\text { Was this period interrupted by your feeling } \\
\text { OK for two months or more? }\end{array}$ & NO & YES \\
\hline B3 & $\begin{array}{l}\text { During this period of feeling depressed } \\
\text { most of the time: }\end{array}$ & & \\
\hline a & Did your appetite change significantly? & NO & YES \\
\hline b & $\begin{array}{l}\text { Did you have trouble sleeping or sleep } \\
\text { excessively? }\end{array}$ & NO & YES \\
\hline c & Did you feel tired or without energy? & NO & YES \\
\hline d & Did you lose your self-confidence? & NO & YES \\
\hline e & $\begin{array}{l}\text { Did you have trouble concentrating or } \\
\text { making decisions? }\end{array}$ & NO & YES \\
\hline f & Did you feel hopeless? & NO & YES \\
\hline & $\begin{array}{l}\text { ARE 2 OR MORE B3 ANSWERS CODED } \\
\text { YES? }\end{array}$ & NO & YES \\
\hline
\end{tabular}

\begin{tabular}{|c|c|c|}
\hline B4 Did the symptoms of depression cause you & NO & YES \\
\cline { 2 - 3 } significant distress or impair & & \\
\hline $\begin{array}{c}\text { your ability to function at work, socially, or } \\
\text { in some other important way? }\end{array}$ & & \\
\hline & & \\
\hline & & \\
\hline
\end{tabular}

\section{O. GENERALIZED ANXIETY DISORDER}

( MEANS : GO TO THE DIAGNOSTIC BOX, CIRCLE NO, AND MOVE TO THE NEXT MODULE)

\begin{tabular}{|l|l|l|l|}
\hline O1 a & Have you worried excessively & NO & YES \\
\hline
\end{tabular}




\begin{tabular}{|c|c|c|c|}
\hline & $\begin{array}{l}\text { or been anxious about several } \\
\text { things }\end{array}$ & & \\
\hline & over the past 6 months? & & \\
\hline \multirow[t]{3}{*}{$\mathrm{b}$} & $\begin{array}{l}\text { Are these worries present most } \\
\text { days? }\end{array}$ & NO & YES \\
\hline & $\begin{array}{l}\text { IS THE PATIENT'S } \\
\text { ANXIETY RESTRICTED } \\
\text { EXCLUSIVELY }\end{array}$ & NO & YES \\
\hline & $\begin{array}{l}\text { TO, OR BETTER } \\
\text { EXPLAINED BY, ANY } \\
\text { DISORDER PRIOR TO THIS } \\
\text { POINT? }\end{array}$ & & \\
\hline \multirow[t]{2}{*}{$\mathrm{O} 2$} & $\begin{array}{l}\text { Do you find it difficult to } \\
\text { control the worries or do they } \\
\text { interfere with }\end{array}$ & NO & YES \\
\hline & $\begin{array}{l}\text { your ability to focus on what } \\
\text { you are doing? }\end{array}$ & & \\
\hline \multirow[t]{3}{*}{$\mathrm{O} 3$} & $\begin{array}{l}\text { FOR THE FOLLOWING, } \\
\text { CODE NO IF THE } \\
\text { SYMPTOMS ARE } \\
\text { CONFINED TO }\end{array}$ & & \\
\hline & $\begin{array}{l}\text { FEATURES OF ANY } \\
\text { DISORDER EXPLORED } \\
\text { PRIOR TO THIS POINT. }\end{array}$ & & \\
\hline & $\begin{array}{l}\text { When you were anxious over } \\
\text { the past } 6 \text { months, did you, } \\
\text { most of the time: }\end{array}$ & & \\
\hline $\mathrm{a}$ & $\begin{array}{l}\text { Feel restless, keyed up or on } \\
\text { edge? }\end{array}$ & NO & YES \\
\hline$b$ & Feel tense? & $\mathrm{NO}$ & YES \\
\hline c & $\begin{array}{l}\text { Feel tired, weak or exhausted } \\
\text { easily? }\end{array}$ & NO & YES \\
\hline d & $\begin{array}{l}\text { Have difficulty concentrating or } \\
\text { find your mind going blank? }\end{array}$ & NO & YES \\
\hline $\mathrm{e}$ & Feel irritable? & $\mathrm{NO}$ & YES \\
\hline \multirow[t]{3}{*}{$\mathrm{f}$} & $\begin{array}{l}\text { Have difficulty sleeping } \\
\text { (difficulty falling asleep, } \\
\text { waking up in the middle }\end{array}$ & NO & YES \\
\hline & $\begin{array}{l}\text { of the night, early morning } \\
\text { wakening or sleeping } \\
\text { excessively)? }\end{array}$ & & \\
\hline & ARE 3 OR MORE O3 & NO & YES \\
\hline
\end{tabular}




\title{
ANSWERS CODED YES?
}

\section{GENERALIZED \\ ANXIETY DISORDER \\ CURRENT}

\author{
E. PANIC DISORDER
}

E1 Have you, on more than one occasion, had spells or attacks when you suddenly felt anxious, frightened, uncomfortable or uneasy, even in situations where most people would not feel that way? Did the spells peak within 10 minutes? CODE YES ONLY IF THE SPELLS PEAK WITHIN 10 MINUTES

NO YES 1

If E1 = NO, CRRCLE NO IN E5 AND SKIP TO F1

E2 At any time in the past, did any of those spells or attacks come on unexpectedly or spontaneously, or occur in an unpredictable or unprovoked manner?

IF E2 $=$ NO, CRRCLE NO IN E5 AND SKIP TO F1

NO YES

2

E3 Have you ever had one such attack followed by a month or more of persistent fear of having another attack, or worries about the consequences of the attack?

IF E3 = NO, CIRCLE NO IN E5 AND SKIP TO F1

NO YES

3

E4 During the worst spell that you can remember :

a Did you have skipping, racing or pounding of your heart ?

b Did you have sweating or clammy hands ?

c Were you trembling or shaking?

d Did you have shortness of breath or difficulty breathing ?

e Did you have a choking sensation or a lump in your throat?

f Did you have chest pain, pressure or discomfort?

$\mathrm{g}$ Did you have nausea, stomach problems or sudden diarrhea?

h Did you feel dizzy, unsteady, lightheaded or faint?

i Did things around you feel strange, unreal, detached or unfamiliar, or did you feel outside of or detached from, part or all of your body?

j Did you fear that you were losing control or going crazy?

$k$ Did you fear that you were dying?

1 Did you have tingling or numbness in parts of your body?

m Did you have hot flashes or chills?

E5 ARE 4 OR MORE E4 ANSWERS CODED YES ? IF ES $=$ NO, SKIP TO E7

E6 In the past month, did you have such attacks repeatedly (2 or more) followed by persistant fear of having another attack?

IF E6 $=$ YES, SKIP TO F 1

E7 ARE 1, 2 OR 3 E4 ANSWERS CODED YES?

\begin{tabular}{|c|c|c|}
\hline No & YES & \\
\hline NO & YES & \\
\hline NO & YES & \\
\hline NO & YES & \\
\hline NO & YES & \\
\hline NO & YES & \\
\hline NO & YES & 1 \\
\hline NO & YES & 1 \\
\hline NO & YES & 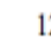 \\
\hline NO & YES & 1 \\
\hline NO & YES & 1 \\
\hline NO & YES & 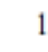 \\
\hline NO & YES & 1 \\
\hline NO & $\begin{array}{l}\text { YES } \\
\text { Panic Disurder } \\
\text { Life time }\end{array}$ & \\
\hline NO & $\begin{array}{l}\text { YES } \\
\text { Panic Disarder } \\
\text { Current }\end{array}$ & 1 \\
\hline NO & $\begin{array}{l}\text { YES } 18 \\
\text { ed Symptom Afacks } \\
\text { Lifetime }\end{array}$ & \\
\hline
\end{tabular}




\title{
Appendix K
}

\section{Human Subjects Training Certificate}

\section{CITI Collaborative Institutional Training Initiative}

\author{
Basic/Refresher Course - Human Subjects Research Curriculum Completion \\ Report
}

\section{Printed on 5/16/2013}

Learner: karen fortuna (username: kfortuna)

Institution: Florida International University

Contact Information Phone: 2157042735

Email:kfortuna@mail.med.upenn.edu

Social/Behavioral Human Research Course: Choose this group to satisfy CITI training requirements for Investigators and staff involved primarily in

Social/Behavioral Research with human subjects.

Stage 1. Basic Course Passed on 05/16/13 (Ref \# 9660463)

\begin{tabular}{|l|c|c|}
\hline Required Modules & $\begin{array}{c}\text { Date } \\
\text { Completed }\end{array}$ & Score \\
\hline Belmont Report and CITI Course Introduction & $05 / 16 / 13$ & $3 / 3(100 \%)$ \\
\hline History and Ethical Principles - SBR & $05 / 16 / 13$ & $5 / 5(100 \%)$ \\
\hline Defining Research with Human Subjects - SBR & $05 / 16 / 13$ & $5 / 5(100 \%)$ \\
\hline $\begin{array}{l}\text { The Regulations and The Social and Behavioral } \\
\text { Sciences - SBR }\end{array}$ & $05 / 16 / 13$ & $4 / 5(80 \%)$ \\
\hline Assessing Risk in Social and Behavioral Sciences - SBR & $05 / 16 / 13$ & $5 / 5(100 \%)$ \\
\hline Informed Consent - SBR & $05 / 16 / 13$ & $5 / 5(100 \%)$ \\
\hline Privacy and Confidentiality - SBR & $05 / 16 / 13$ & $5 / 5(100 \%)$ \\
\hline Research with Prisoners - SBR & $05 / 16 / 13$ & $4 / 4(100 \%)$ \\
\hline Research with Children - SBR & $05 / 16 / 13$ & $4 / 4(100 \%)$ \\
\hline $\begin{array}{l}\text { Research in Public Elementary and Secondary Schools - } \\
\text { SBR }\end{array}$ & $05 / 16 / 13$ & $4 / 4(100 \%)$ \\
\hline International Research - SBR & $05 / 16 / 13$ & $3 / 3(100 \%)$ \\
\hline Internet Research - SBR & $05 / 16 / 13$ & $5 / 5(100 \%)$ \\
\hline Cultural Competence in Research & $05 / 16 / 13$ & $3 / 5(60 \%)$ \\
\hline
\end{tabular}

For this Completion Report to be valid, the learner listed above must be affiliated with a CITI participating institution. Falsified information and unauthorized use of the CITI course site is unethical, and may be considered scientific misconduct by your institution.

Paul Braunschweiger Ph.D.

Professor, University of Miami

Director Office of Research Education

CITI Course Coordinator 
VITA

\section{KAREN FORTUNA}

2012-present Doctoral Candidate in Social Welfare at Florida International University-Miami, FL

2005- 2007

Master of Social Work at University of Pennsylvania-Philadelphia, PA

2003

Bachelor of Arts in Human Services at Western Washington University-Bellingham, WA

\section{PUBLICATIONS AND PRESENTATIONS}

Dillon, F. \& Fortuna (Whiteman), K. (2013). Measurement Equivalence of Short Inventory of Problems-Revised Across Drug Abusing Adult Outpatient Racial Groups. 141st APHA Annual Meeting, Boston, MA. November 2013.

Fortuna (Whiteman), K. (2013). Risk Profile of Older Adults with Co-morbid Depression and Alcohol Use Disorders and Related-health Consequences. Florida International University, Center for Research on U.S. Latino HIV/AIDS and Drug Abuse Brown Bag Series. Miami, FL.

Fortuna (Whiteman), K. (2012). HIV and Older Adults: A Looming Public Health Crisis. Workforce Development: Aging Workshop. Florida International University, Miami, FL.

Fortuna (Whiteman), K. \& Zubritsky, C. (2010). The Behavioral Health Needs of Latino Older Adults. Poster presented at the Institute on Aging Sylvan M. Cohen Annual Retreat. Philadelphia, PA.

Fortuna (Whiteman), K. \& Zubritsky, C. (2010). The Behavioral Health Needs of Latino Older Adults. Lecture presented at the National Council on Aging and the American Society on Aging-Aging in America Conference. Chicago, IL.

Fortuna (Whiteman), K. \& Zubritsky, C. (2009). Bipolar Disorder and Older Adults. Lecture presented at the American Public Health Association Conference. Philadelphia, PA.

Fortuna (Whiteman), K. \& Zubritsky, C. (2009). Assessment of Bipolar Spectrum Disorder in Older Adults and Suicide Prevention. Lecture presented at the Pennsylvania Third Annual Suicide Prevention Conference.

Johnston, S., Davis, L., \& Fortuna (Whiteman), K. (2011). How to Increase CoOccurring Disorders Competence Using a Learning Community Model. Lecture presented at the National Healthcare for the Homeless Council. Washington, DC. 
Rothbard, A., Chhatre, S., Zubritsky, C., \& Fortuna (Whiteman), K. (2010). Effectiveness of a High End Users Program for Persons with Psychiatric Disorders. Poster presented at the American Public Health Association Conference. Denver, CO.

Rothbard, A., Chhatre, S., Zubritsky, C., Fortuna (Whiteman), K., Dettwyler, S., Henry, R.J., \& Smith, M. (2012). Effectiveness of a high end users program for persons with psychiatric disorders. Community Mental Health Journal, 48, 5, 598-603.

Ruggiano, N., Sais, K., \& Whiteman, K. (2014). Older Adult Transportation Needs. 44th Urban Affairs Association Conference. San Antonio, Texas. March 2014.

Ruggiano, N., Whiteman, K., Shtompel N. (in press). "If I Don't Like The Way I Feel With A Certain Drug, I'll Tell Them." Older Adults' Experiences with SelfDetermination and Health Self-advocacy. Journal of Applied Gerontology.

Shtompel, N., Ruggiano, N., Whiteman, K. (in press) Negative feelings and help seeking among older adults with chronic conditions. Journal of Gerontological Social Work.

Zubritsky, C. \& Fortuna, (Whiteman), K. (2010). Memorandum of understanding writing guide. Available at: www.parecovery.org/documents/AgingBH_MOU_Writing_Guide.pdf

Zubritsky, C., Fortuna (Whiteman), K., \& Keogh, B. (2013). Older Adult Mental Health Peer Specialists. University of Medicine and Dentistry, New Jersey, Wellness Coaching Symposium. Scotch Plains, NJ.

Zubritsky, C., Fortuna (Whiteman), K., \& Keogh, B. (2012). Older Adult Mental Health Peer Specialists. American Public Health Association Conference. San Francisco, CA.

Zubritsky, C., Fortuna (Whiteman), K., \& Shumaker, L. (2011). Innovative Approaches to the Advancement of Pennsylvania's Integrated Services Across the State's Aging and Behavioral Health Systems of Care. Poster presented at the American Public Health Association Conference. Washington, DC.

Zubritsky, C. \& Fortuna (Whiteman), K. (2010). Share the Care: Integrating Aging and Mental Health Services. Lecture presented at the American Public Health Association Conference. Denver, CO. 